\title{
Development of Crystal-Tolerant High-Level Waste Glasses
}

Josef Matyáš

John Vienna

Micah Schaible

Carmen Rodriquez
Jarrod Crum

Alyssa Arrigoni

Rachel Tate

December 2010

Pacific Northwest

NATIONAL LABORATORY

Proudly Operated by Battelle Since 1965 


\title{
DISCLAIMER
}

This report was prepared as an account of work sponsored by an agency of the United States Government. Neither the United States Government nor any agency thereof, nor Battelle Memorial Institute, nor any of their employees, makes any warranty, express or implied, or assumes any legal liability or responsibility for the accuracy, completeness, or usefulness of any information, apparatus, product, or process disclosed, or represents that its use would not infringe privately owned rights. Reference herein to any specific commercial product, process, or service by trade name, trademark, manufacturer, or otherwise does not necessarily constitute or imply its endorsement, recommendation, or favoring by the $\mathrm{U}$ nited $\mathrm{S}$ tates G overnment or an y ag ency thereof, or B attelle Memorial Institute. The views and opinions of authors expressed herein do not necessarily state or reflect those of the United States Government or any agency thereof.

\author{
PACIFIC NORTHWEST NATIONAL LABORATORY \\ operated by \\ BATTELLE \\ for the \\ UNITED STATES DEPARTMENT OF ENERGY \\ under Contract DE-AC05-76RL01830
}

Printed in the United States of America
Available to DOE and DOE contractors from the Office of Scientific and Technical Information,
P.O. Box 62, Oak Ridge, TN 37831-0062;
ph: (865) 576-8401
fax: (865) 576-5728
email: reports@adonis.osti.gov

Available to the public from the National Technical Information Service 5301 Shawnee Rd., Alexandria, VA 22312

ph: (800) 553-NTIS (6847)

email: orders@ntis.gov <http://www.ntis.gov/about/form.aspx>

Online ordering: http://www.ntis.gov 


\title{
Development of Crystal-Tolerant High-Level Waste Glasses
}

\author{
Josef Matyáš \\ Jarrod Crum \\ John Vienna \\ Alyssa Arrigoni \\ Micah Schaible \\ Rachel Tate
}

Carmen Rodriquez

December 2010

Prepared for

the U.S. Department of Energy

under Contract DE-AC05-76RL01830

Pacific Northwest National Laboratory

Richland, Washington 99352 



\begin{abstract}
The high-level radioactive waste (HLW) from the Hanford and Savannah River Sites is being vitrified in stable borosilicate glass for long-term storage and disposal. A major concern of the vitrification process is the formation and settling of large spinel crystals in the glass discharge riser of the HLW melter. During numerous and extended melter idling periods from 20 to 100 days, the temperature of molten glass in the riser can drop to $\sim 850^{\circ} \mathrm{C}$ while new feed is being incorporated into the melt. At this temperature, a significant volume of large octahedral crystals of spinel [Fe,Ni, $\mathrm{Mn}, \mathrm{Zn}][\mathrm{Fe}, \mathrm{Cr}]_{2} \mathrm{O}_{4}$ can precipitate in the glass. The settling rate of these crystals is fast enough to form a few $\mathrm{cm}$ thick sludge layer that can partially or completely block the riser during idling, thereby preventing molten glass from discharging during normal operation. This is aggravated by the fact that the spinel sludge cannot be dissolved because the temperature in the riser is relatively low, and the sludge cannot be easily disturbed.

To avoid or minimize the accumulation of crystals, HLW glasses have been formulated with a low liquidus temperature $\left(T_{L}\right)$, or temperature $T_{l}$ at which the equilibrium fraction of spinel crystals in the melt is below $1 \mathrm{vol} \%$, nominally below $1050^{\circ} \mathrm{C}$. However, these constraints cannot prevent the formation, growth, and accumulation of spinel crystals in considerably cooler regions $\left(\sim 850^{\circ} \mathrm{C}\right)$ of the glass discharge riser during melter idling. In addition, the constraints significantly limit the waste loading, which is reflected in a high volume of waste glass, and will result in high capital, production, and disposal costs.
\end{abstract}

We have developed an empirical linear model of spinel settling that can predict crystal accumulation in the riser as a function of glass composition and therefore provide the guidance to formulate crystaltolerant glasses for higher waste loading. 



\section{Summary}

Twenty five glasses were formulated. They were batched from HLW AZ-101 simulant or raw chemicals and melted and tested with a series of tests to elucidate the effect of spinel-forming components (Ni, Fe, Cr, Mn, and $\mathrm{Zn}$ ), $\mathrm{Al}$, and noble metals $\left(\mathrm{Rh}_{2} \mathrm{O}_{3}\right.$ and $\left.\mathrm{RuO}_{2}\right)$ on the accumulation rate of spinel crystals in the glass discharge riser of the high-level waste (HLW) melter. In addition, the processing properties of glasses, such as the viscosity and $T_{L}$, were measured as a function of temperature and composition. Furthermore, the settling of spinel crystals in transparent low-viscosity fluids was studied at room temperature to access the shape factor and hindered settling coefficient of spinel crystals in the Stokes equation.

The experimental results suggest that $\mathrm{Ni}$ is the most troublesome component of all the studied spinelforming components producing settling layers of up to $10.5 \mathrm{~mm}$ in just 20 days in Ni-rich glasses if noble metals or a higher concentration of Fe was not introduced in the glass. The layer of this thickness can potentially plug the bottom of the riser, preventing glass from being discharged from the melter. The noble metals, $\mathrm{Fe}$, and $\mathrm{Al}$ were the components that significantly slowed down or stopped the accumulation of spinel at the bottom. Particles of $\mathrm{Rh}_{2} \mathrm{O}_{3}$ and $\mathrm{RuO}_{2}$, hematite and nepheline, acted as nucleation sites significantly increasing the number of crystals and therefore decreasing the average crystal size. The settling rate of $\leq 10$ - $\mu \mathrm{m}$ crystal size around the settling velocity of crystals was too low to produce thick layers.

The experimental data for the thickness of settled layers in the glasses prepared from AZ-101 simulant were used to build a linear empirical model that can predict crystal accumulation in the riser of the melter as a function of concentration of spinel-forming components in glass. The developed model predicts the thicknesses of accumulated layers quite well, $\mathrm{R}^{2}=0.985$, and can be become an efficient tool for the formulation of the crystal-tolerant HLW glasses for higher waste loading.

A physical modeling effort revealed that the Stokes and Richardson-Zaki equations can be used to adequately predict the accumulation rate of spinel crystals of different sizes and concentrations in the glass discharge riser of HLW melters. The determined shape factor for the glass beads was only $0.73 \%$ lower than the theoretical shape factor for a perfect sphere. The shape factor for the spinel crystals matched the theoretically predicted value to within $10 \%$ and was smaller than that of the beads, given the larger drag force caused by the larger surface area-to-volume ratio of the octahedral crystals. In the hindered settling experiments, both the glass bead and spinel suspensions were found to follow the predictions of the Richardson-Zaki equation with the exponent $n=3.6$ and 2.9 for glass beads and spinel crystals, respectively. 



\section{Acknowledgments}

The authors would like to acknowledge Michael Schweiger and Brian Riley for laboratory assistance, Carol Burnett for supplying glass beads, Pavel Hrma for insightful conversations and help with the mathematical model, and Wayne Cosby for preparation of this document and editorial assistance. Micah Schaible would like to thank the Office of Science for his internship opportunity at Pacific Northwest National Laboratory. This work was funded by the U.S. Department of Energy's Environmental Management Program EM31. Pacific Northwest National Laboratory is operated by Battelle for the U.S. Department of Energy under Contract DE-AC05-76RL01830. 



\section{Acronyms and Abbreviations}

AES

APEL

BL

BRL

CCD

CIAS

EDS

EMSP

HLW

ICP

LOD

LOI

MS

NA

NIST

PNNL

RPD

RSD

SD

SEM

SWRI

XRD atomic emission spectroscopy

Applied Process Engineering Laboratory

baseline glass

below reporting limit

charged coupled device

Clemex Image Analysis Software

energy dispersive spectroscopy

Environmental Management spinel crystals

high-level waste

inductively coupled plasma

loss on drying

loss on ignition

mass spectrometry

not analyzed

National Institute for Standards and Technology

Pacific Northwest National Laboratory

relative percent difference

relative standard deviation

standard deviation

scanning electron microscopy

Southwest Research Institute

$\mathrm{X}$-ray diffraction 



\section{Contents}

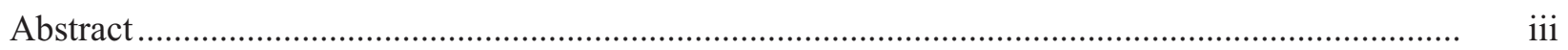

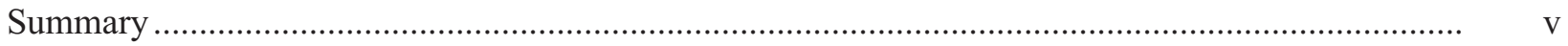

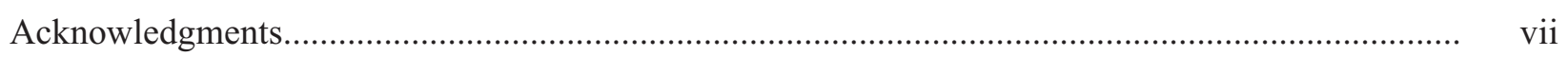

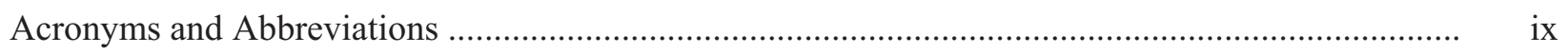

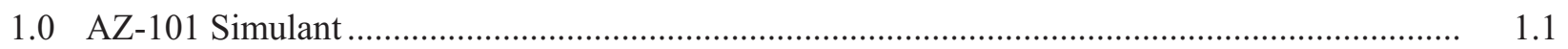

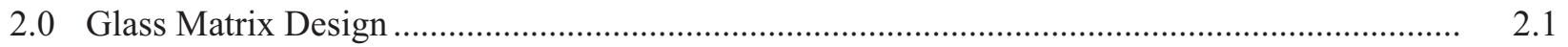

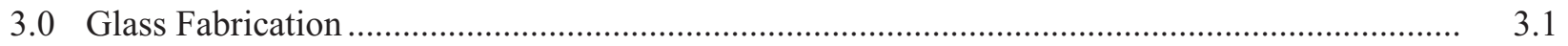

4.0 Analytical Results for AZ-101 Simulant and Selected EMSP Glasses .................................. 4.1

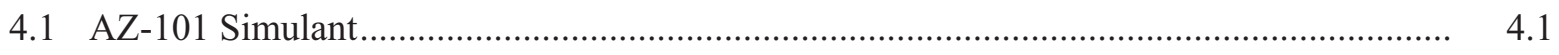

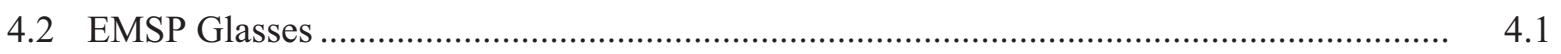

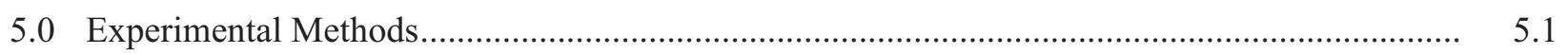

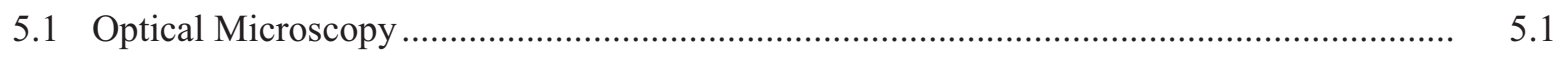

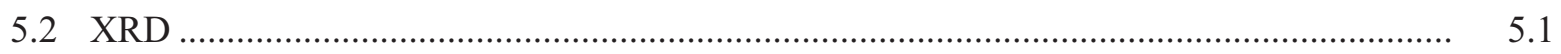

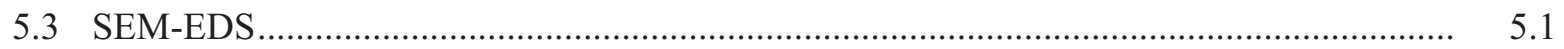

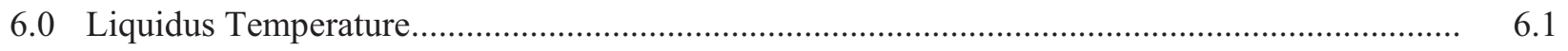

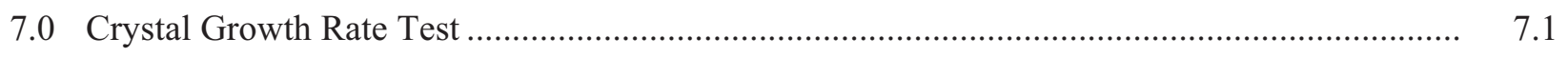

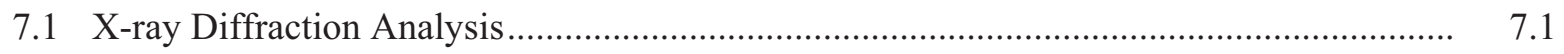

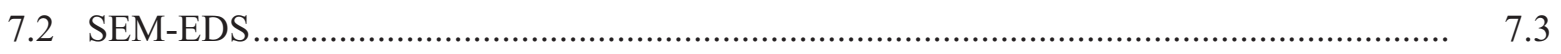

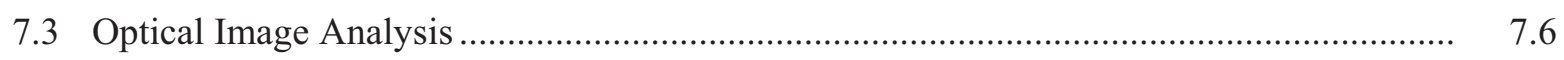

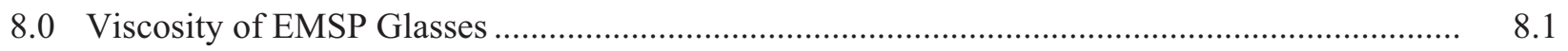

9.0 Double Crucible and High-Chromia Crucible Tests............................................................. 9.1

10.0 Empirical Model of Spinel Crystal Settling........................................................................ 10.1

11.0 Physical Modeling of Particle Settling ................................................................................ 11.1

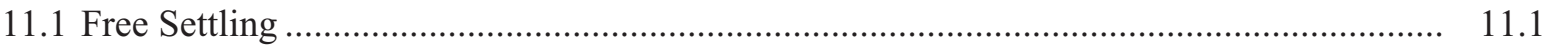

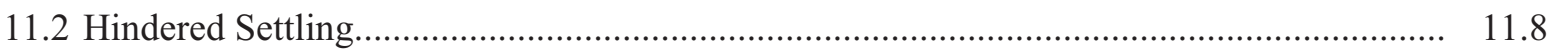




\section{Figures}

1.1. Slurry of AZ-101 Simulant (A), Dried and Ground AZ-101 Simulant (B) ........................... 1.1

7.1. Concentrations of Spinel and Hematite in EMSP-Fe 20 (AZ-101) Glass Heat-Treated at $850^{\circ} \mathrm{C}$ for Different Times.

7.2. Optical Images of Hematite (red platelets) and Spinel (black crystals) that Formed in EMSP-Fe 20 (AZ-101) Glass After Heat-Treatments at $850^{\circ} \mathrm{C}$ for Various Times

7.3. SEM Image of Spinel Crystals (White Particles) and Hematite (Hair-Like Crystals) in EMSP-Fe $20-(A Z-101)$ Glass Heat-Treated at $850^{\circ} \mathrm{C}$ for $12 \mathrm{~h}$

7.4. SEM Image of Spinel Crystals (White Particles) and Hematite (Hair-Like Crystals) in EMSP-Fe $20 / \mathrm{Ni1} .5-(\mathrm{AZ}-101)$ Glass Heat-Treated at $850^{\circ} \mathrm{C}$ for $6 \mathrm{~h}$

7.5. SEM Image of Spinel Crystal in EMSP-Cr0.6-(AZ-101) Glass Heat-Treated at $850^{\circ} \mathrm{C}$ for $3 \mathrm{~h}$

7.6. SEM Image of Spinel Crystal in EMSP-Ni1.5-(AZ-101) Glass Heat-Treated at $850^{\circ} \mathrm{C}$ for $12 \mathrm{~h}$

8.1. Viscosity Curves of EMSP Glasses Prepared from HLW AZ-101 Simulant.

8.2. Viscosity Curves of EMSP Glasses Prepared from Raw Chemicals

9.1. Cross-Section of Double Crucible Assembly (A) and High-Chromia Crucible (B) (redmarked area shows the location of thin-sectioned samples).

9.2. Thickness of Spinel Layer as a Function of Time for Selected EMSP (AZ-101) Glasses that Were Heat-Treated in Double Crucibles at $850^{\circ} \mathrm{C}$

9.3. SEM Images of Spinel Layer in EMSP-Ni 1.5-(AZ-101) Glass After Heat-Treatment at $850^{\circ} \mathrm{C}$ for $92 \mathrm{~h}(\mathrm{~A}), 150 \mathrm{~h}(\mathrm{~B}), 168 \mathrm{~h}(\mathrm{C})$, and $194 \mathrm{~h}(\mathrm{D})$

9.4. Distribution of Average Crystal Size in the Layer of Settled Spinel in EMSP-Ni 1.5(AZ-101) Glass After Heat-Treatment at $850^{\circ} \mathrm{C}$ for $194 \mathrm{~h}$ (9.3D): top $2.6 \mathrm{~mm}$ (A) and bottom $\sim 0.36 \mathrm{~mm}$ (B)

9.5. Change of Average Spinel Crystal Size and Layer Compaction over Time in EMSP-Ni 1.5-(AZ-101) Glass Heat-Treated at $850^{\circ} \mathrm{C}$

9.6. SEM Image of EMSP-Fe20-(AZ-101) Glass Heat-Treated at $850^{\circ} \mathrm{C}$ for 27 Days ....

9.7. Thickness of Spinel Layer as a Function of Time for Selected EMSP (AZ-101) Glasses that Were Heat-Treated in High-Chromia Crucibles at $850^{\circ} \mathrm{C}$

9.8. Thickness of Settled Layer as a Function of Time for Selected EMSP-Ni1.5-(Chem.) Glass Heat-Treated in Double Crucibles at $850^{\circ} \mathrm{C}$

9.9. EMSP-Ni1.5/Al12-(Chem.) Glass After $\sim 310 \mathrm{~h}$ at $850^{\circ} \mathrm{C}$ in Double Crucible; White Features-Spinel Crystals, Dark Features-Nepheline

10.1. Predicted vs. Measured Spinel Layer Thickness

11.1. SEM images of Spherical Soda-Lime-Silica (A, B) and Ba-Ca silicate (C, D) Glass Beads and Their Particle Size Distribution $\left(\mathrm{A}-\rho \sim 2.5 \mathrm{~g} / \mathrm{cm}^{3}, D_{p} \sim 67 \mu \mathrm{m} ; \mathrm{B}-\rho \sim 2.5 \mathrm{~g} / \mathrm{cm}^{3}\right.$, $\left.D_{p} 137 \mu \mathrm{m} ; \mathrm{C}-\rho \sim 4.2 \mathrm{~g} / \mathrm{cm}^{3}, D_{p} \sim 72 \mu \mathrm{m} ; \mathrm{D}-\rho \sim 4.2 \mathrm{~g} / \mathrm{cm}^{3}, D_{p} \sim 161 \mu \mathrm{m}\right)$. 
11.2. SEM Images of Octahedral Spinel Crystals

11.3. Optical Particle-Dynamics-Analyzer.

11.4. Time Sequence Images for Spinel with Edge Lengths $69 \mu \mathrm{m}\left(\mathrm{L}_{1}\right)$ and $79 \mu \mathrm{m}\left(\mathrm{L}_{2}\right)$.

11.5. Linear Fit of Settling Velocity vs. $\left[\left(\rho_{p}-\rho_{f}\right) g R^{2}\right] / \eta$ for Glass Beads

11.6. Linear Fit of Settling Velocity vs. $\left[\left(\rho_{p}-\rho_{f}\right) g R^{2}\right] / \eta$ for Single and Agglomerated Spinel Crystals.

11.7. Two (A) and Multiple-Particle (B) Agglomerates of Spinel Crystals .....

11.8. Richardson-Zaki Hindered Settling Coefficient vs. Volume Fraction of Solids

11.9. Image Threshold Analysis of Tested Suspensions (particle voids — blue; glass beads and crystals - green): A) 1 vol\% of 150 - to $180-\mu \mathrm{m}$ Glass Beads in 4.84 Pa.s oil, B) 1 vol\% of 149- to 212- $\mu \mathrm{m}$ Spinel Crystals in 5.08 Pa.s Oil, and C) Detail of Rectangle from Image B ... 11.10

11.10. Settling Front Movement for 1-vol\% Silicone Oil Suspensions of Glass Beads and Spinel Crystals: A) 150 - to $180-\mu \mathrm{m}$ Glass Beads in 4.84 Pa.s oil, B) 149- to 212- $\mu \mathrm{m}$ Spinel Crystals in 5.08 Pa.s Oil

11.11. Change of Particle Void Fraction over Time and Distance and Determined Positions of Settling Front for 1 vol\% of 149- to 212- $\mu \mathrm{m}$ Spinel Crystals in 5.08 Pa.s Oil

11.12. Change of Particle Void Fraction over Time and Distance and Determined Positions of Settling Front for 1-vol\% Oil (4.84 Pa.s) Suspension of 150- to 180- $\mu \mathrm{m}$ Glass Beads

11.13. Distance vs. Time for 1 vol\% Silicone Oil Suspensions of Glass Beads and Spinel Crystals: A) 150 - to $180-\mu \mathrm{m}$ Glass Beads in 4.84 Pa.s Oil, B) 67- to 80- $\mu \mathrm{m}$ Glass Beads in 4.84 Pa.s Oil, C) 150- to $180-\mu \mathrm{m}$ Glass Beads in 0.98 Pa.s Oil, and D) 149 - to $212-\mu \mathrm{m}$ Spinel Crystals in 5.08 Pa.s Oil

11.14. Plot of $\log \left(1-\mathrm{C}_{0}\right)$ vs. $\log \left(\mathrm{v}^{\prime \prime} / \mathrm{v}\right)$. Slopes of fitted lines correspond to Richardson-Zaki exponent $n$. 


\section{Tables}

1.1. Composition of HLW AZ-101 Simulant in Mass Fraction of Oxides and Halogens ............... 1.2

2.1. Glass Property and Component Constraints to Formulate a Baseline Glass Composition........ 2.1

2.2. Concentration Variation of Noble Metals, Cr, Ni, Fe, Zn, Mn, and Al, in Hanford HLW in Mass Fraction of Oxides.

2.3. Composition of Matrix Glasses EMSP-XX-(AZ-101) in Mass Fraction of Oxides and Halogens 2.2

2.4. Composition of Matrix Glasses EMSP-XX-(Chem.) in Mass Fraction of Oxides and Halogens ....

2.5. Predicted Properties of Matrix Glasses Prepared from AZ-101 Simulant and Additives

2.6. Predicted Properties of Matrix Glasses Prepared from Raw Chemicals

4.1. Analyzed Composition of AZ-101 Simulant Compared with Target Composition of Simulant from Literature.

4.2. Analyzed Composition of Baseline Glass EMSP-BL-(AZ-101)-1M, After First Melt, Compared with Target Values for Designed BL Glass

4.3. Analyzed Composition of Baseline Glass EMSP-BL-(AZ-101)-1M, after First Melt, Compared with Analyzed Composition of Baseline Glass EMSP-BL-(AZ-101)-7D, after 7 days at $850^{\circ} \mathrm{C}$ (Double Crucible Test).

4.4. Analyzed Composition of EMSP-Cr0.6-(AZ-101)-1M) Glass after First Melt Compared with Target Values for Designed Cr0.6 Glass

4.5. Analyzed Composition of EMSP-Cr0.6-(AZ-101)-1M Glass after First Melt Compared with Analyzed Composition of EMSP-Cr0.6 (AZ-101)-7D Glass after 7 days at $850^{\circ} \mathrm{C}$ (Double Crucible Test)

4.6. Analyzed Composition of EMSP-Ni1.5-1M Glass after First Melt Compared with Target Values for Designed Ni1.5 Glass

4.7. Analyzed Composition of EMSP-Ni1.5-1M Glass after First Melt Compared with Analyzed Composition of EMSP-Ni1.5-7D Glass after 7 Days at $850^{\circ} \mathrm{C}$ (Double Crucible Test).

4.8. Analyzed Composition of EMSP-Fe20-1M Glass after First Melt Compared with Target Values for Designed Fe20 Glass

4.9. Analyzed Composition of EMSP-Fe20-1M Glass after First Melt Compared with Analyzed Composition of EMSP-Fe20-8D Glass after 8 Days at $850^{\circ} \mathrm{C}$ (Double Crucible Test)...

4.10. Analyzed Composition of EMSP-Ni1.5 (Chem.)-1M Glass after First Melt Compared with Target Values for Designed Ni1.5 Glass .....

4.11. Analyzed Composition of Baseline Glass EMSP-Ni1.5-(Chem.)-1M, After First Melt, Compared with Analyzed Composition of Baseline Glass EMSP-Ni1.5-7D, After 7 Days at $850^{\circ} \mathrm{C}$ (Double Crucible Test).

6.1. Determined $\mathrm{T}_{\mathrm{L}}$ 's 
7.1. Concentration of Spinel in wt $\%$ at Different Temperatures and Times for EMSP-BL-(AZ101)

7.2. Concentration of Spinel in wt $\%$ at Different Temperatures and Times for EMSP-Cr0.6(AZ-101).

7.3. Concentration of Spinel in wt $\%$ at Different Temperatures and Times for EMSP-Cr1.2(AZ-101).

7.4. Concentration of Spinel in wt $\%$ at Different Temperatures and Times for EMSP-Fe20(AZ-101).

7.5. Concentration of Spinel in wt $\%$ at Different Temperatures and Times for EMSPFe20/Ni1.5- (AZ-101)

7.6. Concentration of Spinel in wt $\%$ at Different Temperatures and Times for EMSP-Ni1.07(AZ-101)

7.7. Concentration of Spinel in wt $\%$ at Different Temperatures and Times for EMSP-Ni1.5(AZ-101)

7.8. Crystal Size of Spinel Crystals in $\mu \mathrm{m}$ for Various AZ-101 Glasses that Were Heat-Treated at $850^{\circ} \mathrm{C}$ for Different Times

8.1. Calculated Glass Viscosities at 850,1050 , and $1200^{\circ} \mathrm{C}$

9.1. Concentration of $\mathrm{Cr}, \mathrm{Fe}$, and $\mathrm{Ni}$ in Glass in Mass \% of Oxides at the Center of the CrossSectioned Crucible $\sim 2 \mathrm{~mm}$ from the Left (L) and the Right (R) Walls and in the Middle (M). Glasses were Heat-Treated in High-Chromia Crucibles at $850^{\circ} \mathrm{C}$ for 4 Days.

9.2. The Effect of $\mathrm{Ru}_{2} \mathrm{O}_{3}$ on Thickness of Settled Layers, Crystal Sizes, and Crystal Number Densities for Ni-rich (Chem) Glasses

9.3. The Effect of $\mathrm{Al}_{2} \mathrm{O}_{3}$ and $\mathrm{Fe}_{2} \mathrm{O}_{3}$ on Thickness of Settled Layers, Crystal Sizes, and Crystal Number Densities for Ni-rich (Chem) Glasses

9.4. The Effect of $\mathrm{Al}_{2} \mathrm{O}_{3}$ and $\mathrm{Fe}_{2} \mathrm{O}_{3}$ on Thickness of Settled Layers, Crystal Sizes, and Crystal Number Densities for Glasses Prepared from Chemicals

10.1. Component Coefficients Calculated with PNNL Model

11.1. Settling Velocities and Shape Factors for Glass Beads of Different Size and Density in Different Viscosity Oils.

11.2. Characteristics of Particle Suspensions for the Hindered Settling Experiments 



\subsection{AZ-101 Simulant}

AZ-101 simulant of pretreated high-level waste (HLW) from Tank 241-AZ-101 was selected for the laboratory study of spinel formation and accumulation in the joule-heated ceramic melters. The vendor, Optima Chemical Co., supplied more than 11,000 L of the AZ-101 simulant for prototypic testing and analytical studies of gas retention and release in simulant with an anti-foam agent. This simulant did not contain any noble metals because they do not catalyze an increased gas-holdup characteristic of the simulant. Approximately $155 \mathrm{~L}$ of this simulant was used for a crystal-tolerant, glass-development study.

The AZ-101 simulant was mixed in a 1000-L tote for approximately 1 hour with a diaphragm pump and air sparger to make sure of a uniform distribution of undissolved solids. Homogenized simulant was transferred to 2-L plastic bottles using the peristaltic pump with the diaphragm pump still running to restrict the settling of solids in the tote. A total of 75 bottles was collected, including fifteen $250-\mathrm{mL}$ samples to determine the loss on drying (LOD). These samples were collected every time five bottles were filled with the simulant. The LOD varied from 23.97 to $24.10 \mathrm{wt} \%$, suggesting a uniform distribution of solids in the AZ-101 simulant. The simulant in plastic bottles was mixed using a roller, transferred into the stainless steel pans, allowed to oven-dry at $105^{\circ} \mathrm{C}$ for 4 days, and crushed and ground for 2 min using a tungsten carbide mill before being incorporated into the borosilicate glasses. Figure 1.1 shows AZ-101 simulant slurry and simulant after drying and grinding.

A

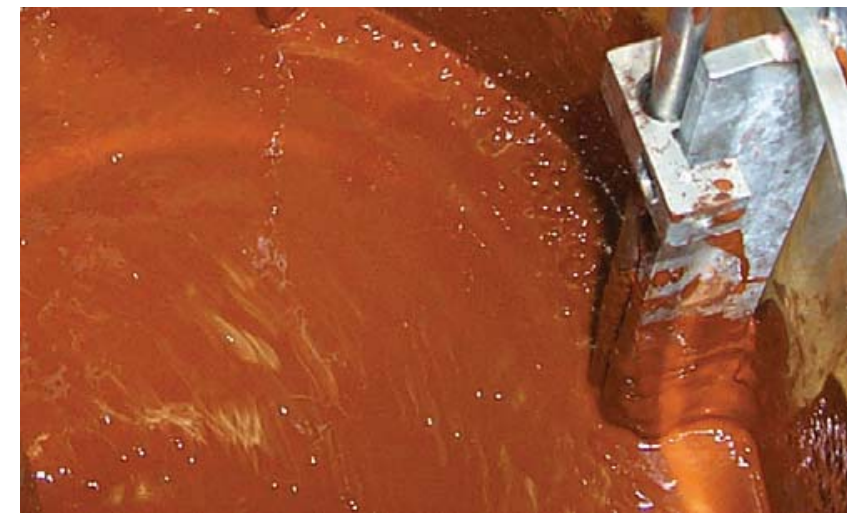

B

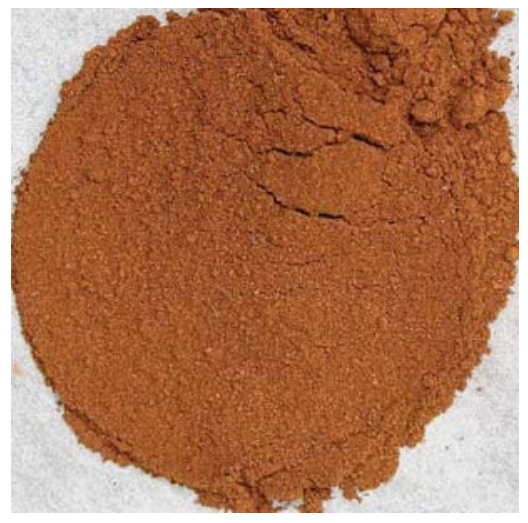

Figure 1.1. Slurry of AZ-101 Simulant (A), Dried and Ground AZ-101 Simulant (B)

The composition of the AZ-101 simulant (Eibling et al. ${ }^{\text {(a) }}$ 2003, Appendix F) was derived from a combination of AZ-101 waste, based on the characterization of a sample of actual AZ-101 sludge, with a portion of cesium ion exchange concentrate, which comes from the return of radioactive cesium from the sludge supernatant back to the HLW. Table 1.1 shows the composition of HLW AZ-101 simulant that was used to develop the crystal tolerant glass. The chemical compositions of the raw materials were converted to oxide and halogen concentrations that will remain in the glass.

(a) Russell E. Eibling, Ray F. Schumacher, and Erich K. Hansen. 2003. Development of Simulants to Support Mixing Tests for High Level Waste and Low Activity Waste. SRT-RPP-2003-00098, REV. 0. Westinghouse Savannah River Company. Savannah River Site, Aiken, SC. 
Table 1.1. Composition of HLW AZ-101 Simulant in Mass Fraction of Oxides and Halogens

\begin{tabular}{llllllll}
\hline \multicolumn{2}{c}{ Component } & \multicolumn{2}{r}{ Component } & \multicolumn{2}{c}{ Component } & \multicolumn{2}{c}{ Component } \\
\hline $\mathrm{Al}_{2} \mathrm{O}_{3}$ & 0.2263 & $\mathrm{Fe}_{2} \mathrm{O}_{3}$ & 0.3999 & $\mathrm{Rh}_{2} \mathrm{O}_{3}$ & $\mathrm{NA}$ & $\mathrm{Cl}$ & 0.0006 \\
$\mathrm{~B}_{2} \mathrm{O}_{3}$ & 0.0159 & $\mathrm{~K}_{2} \mathrm{O}$ & 0.0095 & $\mathrm{RuO}_{2}$ & $\mathrm{NA}$ & $\mathrm{Ce}_{2} \mathrm{O}_{3}$ & 0.0056 \\
$\mathrm{BaO}$ & 0.0026 & $\mathrm{MgO}$ & 0.0036 & $\mathrm{SiO}_{2}$ & 0.0467 & $\mathrm{CoO}$ & 0.0003 \\
$\mathrm{CaO}$ & 0.0158 & $\mathrm{MnO}$ & 0.0097 & $\mathrm{SO}_{3}$ & 0.0023 & $\mathrm{CuO}$ & 0.0011 \\
$\mathrm{CdO}$ & 0.0178 & $\mathrm{Na}_{2} \mathrm{O}$ & 0.0786 & $\mathrm{TiO}_{2}$ & 0.0008 & $\mathrm{La}_{2} \mathrm{O}_{3}$ & 0.0061 \\
$\mathrm{Cr}_{2} \mathrm{O}_{3}$ & 0.0047 & $\mathrm{NiO}$ & 0.0175 & $\mathrm{ZnO}$ & 0.0006 & $\mathrm{Nd}_{2} \mathrm{O}_{3}$ & 0.005 \\
$\mathrm{~F}$ & 0.0002 & $\mathrm{P}_{2} \mathrm{O}_{5}$ & 0.0088 & $\mathrm{ZrO}_{2}$ & 0.1148 & $\mathrm{SnO}_{2}$ & 0.0027 \\
\hline \multicolumn{7}{l}{ NA: Noble metals were not added. } \\
\hline
\end{tabular}




\subsection{Glass Matrix Design}

The baseline glass composition (Environmental Management spinel crystals-baseline glass [EMSPBL]) was formulated with the composition of AZ-101 simulant given in Table 1.1. The additive composition and waste loading were adjusted by maximizing the waste loading while satisfying the glass property and component constraints that are listed in Table 2.1. The additives used were $\mathrm{B}_{2} \mathrm{O}_{3}, \mathrm{Li}_{2} \mathrm{O}$, $\mathrm{Na}_{2} \mathrm{O}$, and $\mathrm{SiO}_{2}$. To study the effect of noble metals ( $\mathrm{Rh}$ and $\mathrm{Ru}$ ), $\mathrm{Cr}, \mathrm{Ni}, \mathrm{Fe}, \mathrm{Zn}, \mathrm{Mn}$, and $\mathrm{Al}$ on spinel concentration, crystal size, and settling rate, the composition of designed glasses was varied one or two components-at-a-time from the baseline glass composition while proportionally decreasing the concentration of all other components. The concentration of $\mathrm{Rh}_{2} \mathrm{O}_{3}, \mathrm{RuO}_{2}, \mathrm{Cr}_{2} \mathrm{O}_{3}, \mathrm{NiO}, \mathrm{Fe}_{2} \mathrm{O}_{3}, \mathrm{ZnO}$, $\mathrm{MnO}$, and $\mathrm{Al}_{2} \mathrm{O}_{3}$ in matrix glasses was varied to encompass the variation of these components in Hanford HLW. ${ }^{\text {(a) }}$ Table 2.2 summarizes the concentration ranges for the components in Hanford HLW. Twenty five glasses were fabricated. Twelve glasses were prepared from AZ-101 simulant and additives [EMSPXX-(AZ-101)], and 13 from raw chemicals [EMSP-XX-(Chem.)]. Table 2.3 and Table 2.4 show the compositions of tested glasses that were prepared from AZ-101 simulant and from raw chemicals, including the baseline glasses. Table 2.5 and Table 2.6 summarize their predicted properties. ${ }^{(b)}$

Table 2.1. Glass Property and Component Constraints to Formulate a Baseline Glass Composition

\begin{tabular}{llcc}
\hline \multicolumn{1}{c}{ Constraints } & \multicolumn{1}{c}{ Purpose } & Unit & Range \\
\hline $\mathrm{T}_{\mathrm{L}}$ (spinel) & Crystals in the melter & ${ }^{\circ} \mathrm{C}$ & $\leq 1200$ \\
$\mathrm{~T}_{\mathrm{L}}$ (zircon) & Crystals in the melter & ${ }^{\circ} \mathrm{C}$ & $\leq 1200$ \\
$\mathrm{~T}_{1}$ (spinel) & Crystals in the melter & ${ }^{\circ} \mathrm{C}$ & $\leq 1100$ \\
$\eta$ at $1150^{\circ} \mathrm{C}$ & Processability & $\mathrm{Pa} \cdot \mathrm{s}$ & $4-10$ \\
$\varepsilon$ at $1150^{\circ} \mathrm{C}$ & Processability & $\mathrm{S} / \mathrm{m}$ & $10-100$ \\
$\mathrm{PCT}-\mathrm{B}$ & Glass durability & $\mathrm{g} / \mathrm{m}^{2}$ & $\leq 4.18$ \\
$\mathrm{PCT}-\mathrm{Li}$ & Glass durability & $\mathrm{g} / \mathrm{m}^{2}$ & $\leq 2.39$ \\
$\mathrm{PCT}_{-} \mathrm{Na}$ & Glass durability & $\mathrm{g} / \mathrm{m}^{2}$ & $\leq 3.34$ \\
$\mathrm{TCLP} \mathrm{Cd}^{2}$ & $\mathrm{mg} / \mathrm{L}$ & $\leq 0.48$ \\
$\mathrm{SiO}_{2} /\left(\mathrm{SiO}{ }_{2}+\mathrm{Na}_{2} \mathrm{O}+\mathrm{Al}_{2} \mathrm{O}_{3}\right)$ & Delisting & - & $\geq 0.60$ \\
$\mathrm{RuO}_{2}+\mathrm{Rh}_{2} \mathrm{O}_{3}+\mathrm{PdO}^{2}$ & Nepheline, durability & $\mathrm{Wt} \%$ & $\leq 0.25$ \\
$\mathrm{Al}_{2} \mathrm{O}_{3}$ & Crystals in the melter & $\mathrm{Wt} \%$ & $\leq 20$ \\
$\mathrm{~B}_{2} \mathrm{O}_{3}$ & Model validity & $\mathrm{Wt} \%$ & $5-20$ \\
$\mathrm{Cr}_{2} \mathrm{O}_{3}$ & Model validity & $\mathrm{Wt} \%$ & $\leq 1$ \\
$\mathrm{~F}$ & Crystals in the melter & $\mathrm{Wt} \%$ & $\leq 2$ \\
$\mathrm{Fe}_{2} \mathrm{O}_{3}$ & Phase separation & $\mathrm{Wt} \%$ & $\leq 20$ \\
$\mathrm{~K}_{2} \mathrm{O}$ & Model validity & $\mathrm{Wt} \%$ & $\leq 6$ \\
$\mathrm{Li}_{2} \mathrm{O}$ & Model validity & $\mathrm{Wt} \%$ & $\leq 4$ \\
$\mathrm{Na}_{2} \mathrm{O}$ & Model validity & $\mathrm{Wt} \%$ & $\leq 20$ \\
$\mathrm{P}_{2} \mathrm{O}_{5}$ & Model validity & $\mathrm{Wt} \%$ & $\leq 2.5$ \\
$\mathrm{SiO}_{2}$ & Phase separation & $\geq 30$ \\
$\mathrm{SO}_{3}$ & Model validity & $\leq 0.8$ \\
\hline & & & \\
\hline & & & \\
\hline
\end{tabular}

(a) HLW composition from MRQ06-0069HCP $1 \& 2$.

(b) E Lee, Dynamic (G2) Flowsheet Assessment of the Effect of M-12 Modifications on Pretreatment Capacity, 24590-WTP-RPT-PO-07-002, Rev 0, April 2007. 
Table 2.2. Concentration Variation of Noble Metals, Cr, Ni, Fe, Zn, Mn, and Al, in Hanford HLW in Mass Fraction of Oxides

\begin{tabular}{lcc}
\hline Component & Minimum & Maximum \\
\hline $\mathrm{Rh}_{2} \mathrm{O}_{3}$ & $1.1 \mathrm{E}-08$ & 0.0004 \\
$\mathrm{RuO}_{2}$ & $2.8 \mathrm{E}-06$ & 0.0024 \\
$\mathrm{Cr}_{2} \mathrm{O}_{3}$ & 0.0027 & 0.0584 \\
$\mathrm{NiO}$ & 0.0012 & 0.0351 \\
$\mathrm{Fe}_{2} \mathrm{O}_{3}$ & 0.0140 & 0.5244 \\
$\mathrm{ZnO}$ & 0.0005 & 0.0181 \\
$\mathrm{MnO}$ & 0.0018 & 0.0668 \\
$\mathrm{Al}_{2} \mathrm{O}_{3}$ & 0.0704 & 0.7350 \\
\hline
\end{tabular}

Table 2.3. Composition of Matrix Glasses EMSP-XX-(AZ-101) in Mass Fraction of Oxides and Halogens

\begin{tabular}{|c|c|c|c|c|c|c|c|c|c|c|c|c|}
\hline Component & $\mathrm{BL}$ & Cr0.6 & $\mathrm{Cr} 1.2$ & Ni1.07 & Ni1.5 & $\mathrm{Ni} 1.5 / \mathrm{nm}^{\mathrm{a}}$ & $\mathrm{Fe} 20$ & Fe20/Ni1.5 & Mn1 & $\mathrm{Mn} 2.5$ & $\mathrm{Zn} 0.6$ & Ni1.2/Al12 \\
\hline $\mathrm{Al}_{2} \mathrm{O}_{3}$ & 0.0821 & 0.0817 & 0.0813 & 0.0817 & 0.0814 & 0.0814 & 0.0768 & 0.0760 & 0.0816 & 0.0803 & 0.0816 & 0.1200 \\
\hline $\mathrm{B}_{2} \mathrm{O}_{3}$ & 0.0799 & 0.0796 & 0.0791 & 0.0796 & 0.0792 & 0.0792 & 0.0748 & 0.0739 & 0.0794 & 0.0782 & 0.0794 & 0.0758 \\
\hline $\mathrm{BaO}$ & 0.0009 & 0.0009 & 0.0009 & 0.0009 & 0.0009 & 0.0009 & 0.0008 & 0.0008 & 0.0009 & 0.0009 & 0.0009 & 0.0009 \\
\hline $\mathrm{CaO}$ & 0.0057 & 0.0057 & 0.0056 & 0.0057 & 0.0057 & 0.0056 & 0.0053 & 0.0053 & 0.0057 & 0.0056 & 0.0057 & 0.0054 \\
\hline $\mathrm{CdO}$ & 0.0065 & 0.0065 & 0.0064 & 0.0065 & 0.0064 & 0.0064 & 0.0061 & 0.0060 & 0.0065 & 0.0064 & 0.0065 & 0.0062 \\
\hline $\mathrm{Cr}_{2} \mathrm{O}_{3}$ & 0.0017 & 0.0060 & 0.0120 & 0.0017 & 0.0017 & 0.0017 & 0.0016 & 0.0016 & 0.0017 & 0.0017 & 0.0017 & 0.0016 \\
\hline F & 0.0001 & 0.0001 & 0.0001 & 0.0001 & 0.0001 & 0.0001 & 0.0001 & 0.0001 & 0.0001 & 0.0001 & 0.0001 & 0.0001 \\
\hline $\mathrm{Fe}_{2} \mathrm{O}_{3}$ & 0.1451 & 0.1445 & 0.1436 & 0.1445 & 0.1438 & 0.1438 & 0.2000 & 0.2000 & 0.1442 & 0.1420 & 0.1443 & 0.1377 \\
\hline $\mathrm{K}_{2} \mathrm{O}$ & 0.0034 & 0.0034 & 0.0034 & 0.0034 & 0.0034 & 0.0034 & 0.0032 & 0.0031 & 0.0034 & 0.0033 & 0.0034 & 0.0032 \\
\hline $\mathrm{Li}_{2} \mathrm{O}$ & 0.0199 & 0.0198 & 0.0197 & 0.0198 & 0.0197 & 0.0197 & 0.0186 & 0.0184 & 0.0198 & 0.0195 & 0.0198 & 0.0189 \\
\hline $\mathrm{MgO}$ & 0.0013 & 0.0013 & 0.0013 & 0.0013 & 0.0013 & 0.0013 & 0.0012 & 0.0012 & 0.0013 & 0.0013 & 0.0013 & 0.0012 \\
\hline $\mathrm{MnO}$ & 0.0035 & 0.0035 & 0.0035 & 0.0035 & 0.0035 & 0.0035 & 0.0033 & 0.0032 & 0.0100 & 0.025 & 0.0035 & 0.0033 \\
\hline $\mathrm{Na}_{2} \mathrm{O}$ & 0.1866 & 0.1858 & 0.1847 & 0.1858 & 0.1850 & 0.1849 & 0.1746 & 0.1726 & 0.1854 & 0.1826 & 0.1855 & 0.1771 \\
\hline $\mathrm{NiO}$ & 0.0064 & 0.0064 & 0.0063 & 0.0107 & 0.0150 & 0.0150 & 0.0060 & 0.0150 & 0.0064 & 0.0063 & 0.0064 & 0.0150 \\
\hline $\mathrm{P}_{2} \mathrm{O}_{5}$ & 0.0032 & 0.0032 & 0.0032 & 0.0032 & 0.0032 & 0.0032 & 0.0030 & 0.0030 & 0.0032 & 0.0031 & 0.0032 & 0.0030 \\
\hline $\mathrm{SiO}_{2}$ & 0.4031 & 0.4014 & 0.3989 & 0.4014 & 0.3996 & 0.3995 & 0.3772 & 0.3729 & 0.4005 & 0.3944 & 0.4008 & 0.3825 \\
\hline $\mathrm{SO}_{3}$ & 0.0008 & 0.0008 & 0.0008 & 0.0008 & 0.0008 & 0.0008 & 0.0007 & 0.0007 & 0.0008 & 0.0008 & 0.0008 & 0.0008 \\
\hline $\mathrm{TiO}_{2}$ & 0.0003 & 0.0003 & 0.0003 & 0.0003 & 0.0003 & 0.0003 & 0.0003 & 0.0003 & 0.0003 & 0.0003 & 0.0003 & 0.0003 \\
\hline $\mathrm{ZnO}$ & 0.0002 & 0.0002 & 0.0002 & 0.0002 & 0.0002 & 0.0002 & 0.0002 & 0.0002 & 0.0002 & 0.0002 & 0.006 & 0.0002 \\
\hline $\mathrm{ZrO}_{2}$ & 0.0416 & 0.0414 & 0.0412 & 0.0414 & 0.0412 & 0.0412 & 0.0389 & 0.0385 & 0.0413 & 0.0407 & 0.0414 & 0.0395 \\
\hline $\mathrm{Cl}$ & 0.0002 & 0.0002 & 0.0002 & 0.0002 & 0.0002 & 0.0002 & 0.0002 & 0.0002 & 0.0002 & 0.0002 & 0.0002 & 0.0002 \\
\hline $\mathrm{Ce}_{2} \mathrm{O}_{3}$ & 0.0020 & 0.0020 & 0.0020 & 0.0020 & 0.0020 & 0.0020 & 0.0019 & 0.0019 & 0.0020 & 0.0020 & 0.0020 & 0.0019 \\
\hline $\mathrm{CoO}$ & 0.0001 & 0.0001 & 0.0001 & 0.0001 & 0.0001 & 0.0001 & 0.0001 & 0.0001 & 0.0001 & 0.0001 & 0.0001 & 0.0001 \\
\hline $\mathrm{CuO}$ & 0.0004 & 0.0004 & 0.0004 & 0.0004 & 0.0004 & 0.0004 & 0.0004 & 0.0004 & 0.0004 & 0.0004 & 0.0004 & 0.0004 \\
\hline $\mathrm{La}_{2} \mathrm{O}_{3}$ & 0.0022 & 0.0022 & 0.0022 & 0.0022 & 0.0022 & 0.0022 & 0.0021 & 0.0020 & 0.0022 & 0.0022 & 0.0022 & 0.0021 \\
\hline $\mathrm{Nd}_{2} \mathrm{O}_{3}$ & 0.0018 & 0.0018 & 0.0018 & 0.0018 & 0.0018 & 0.0018 & 0.0017 & 0.0017 & 0.0018 & 0.0018 & 0.0018 & 0.0017 \\
\hline $\mathrm{SnO}_{2}$ & 0.0010 & 0.0010 & 0.0010 & 0.0010 & 0.0010 & 0.0010 & 0.0009 & 0.0009 & 0.0010 & 0.0010 & 0.0010 & 0.0009 \\
\hline Total & 1.0000 & 1.0000 & 1.0000 & 1.0000 & 1.0000 & 0.9997 & 1.0000 & 1.0000 & 1.0000 & 1.0000 & 1.0000 & 1.0000 \\
\hline
\end{tabular}




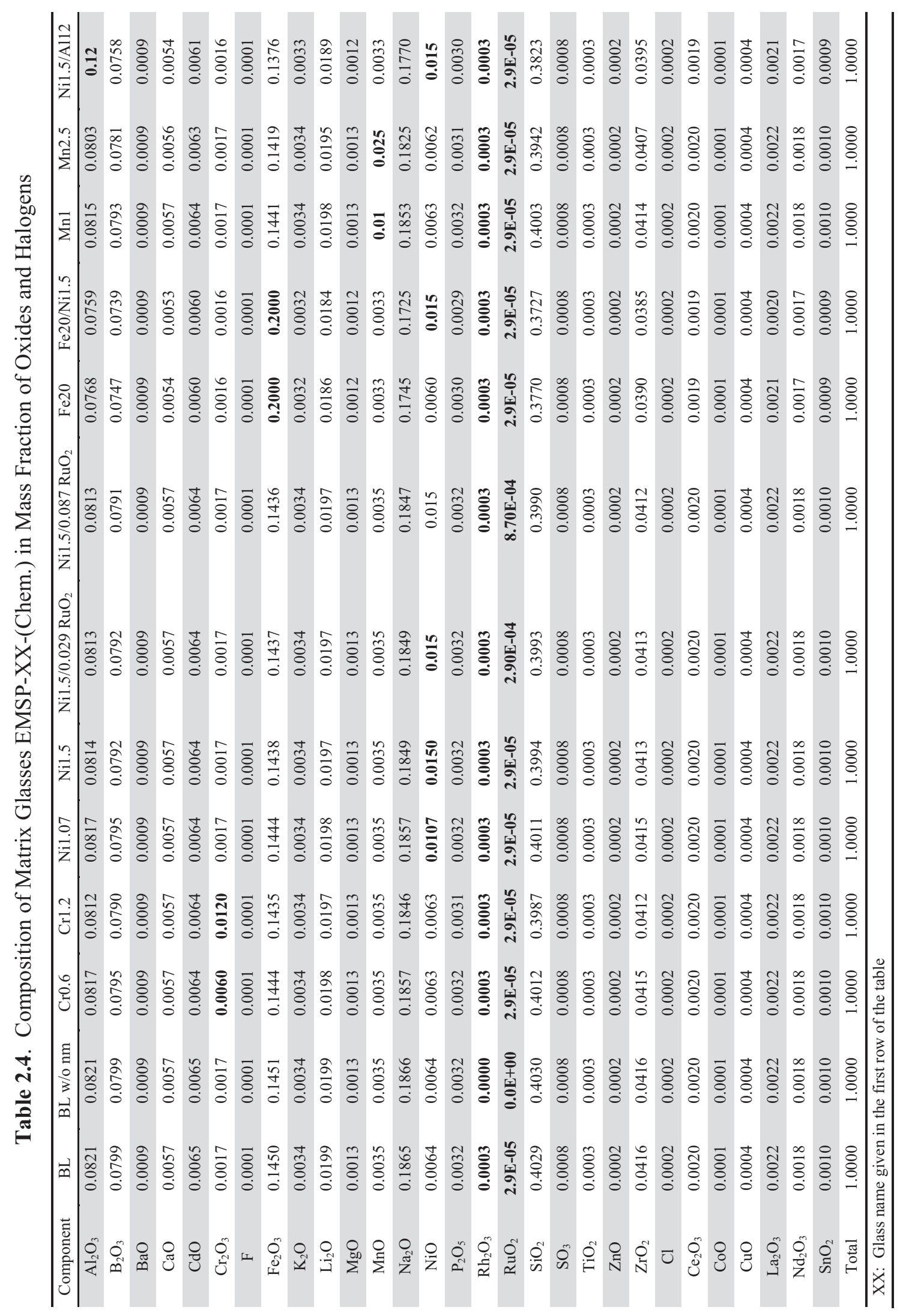




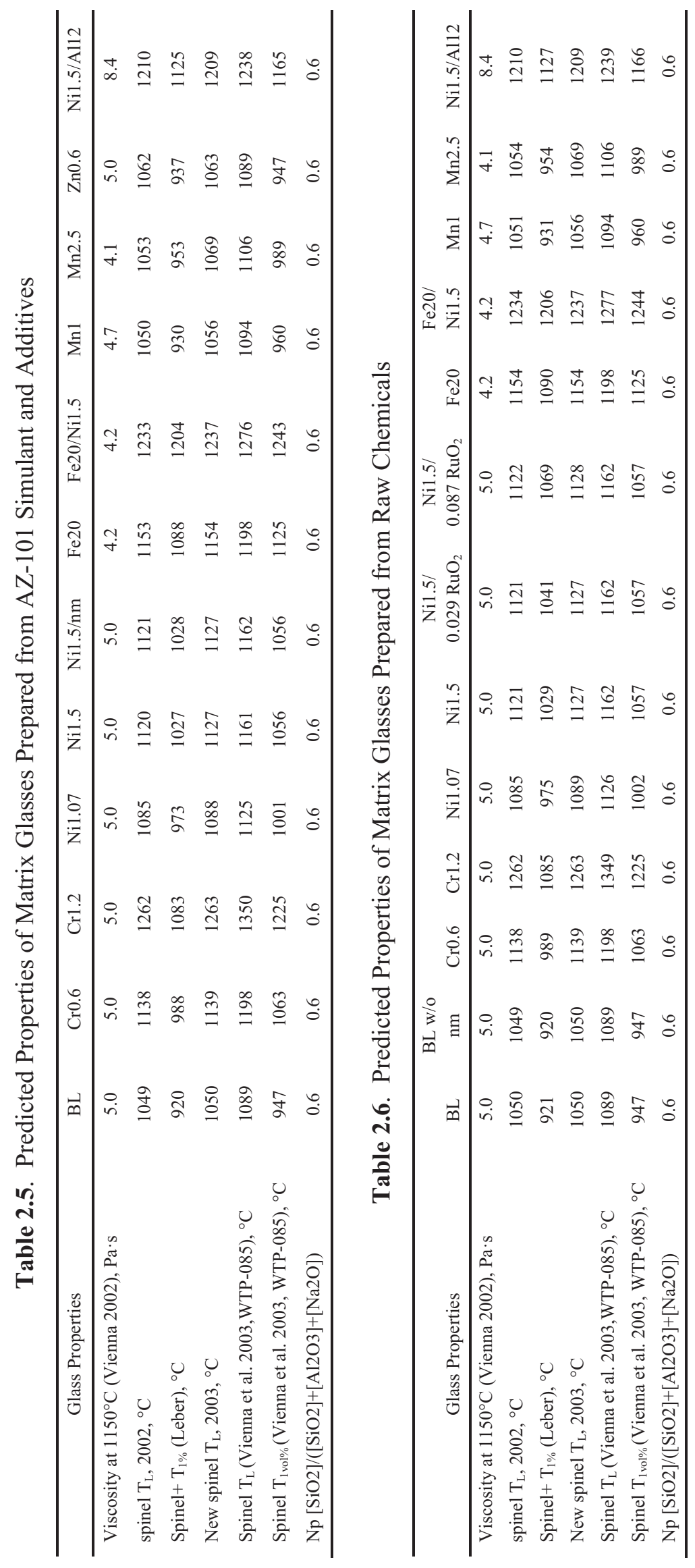




\subsection{Glass Fabrication}

Glass fabrication was performed according to the Pacific Northwest National Laboratory (PNNL) procedure GDL-GBM ${ }^{(\mathbf{a})}$ for glass batching and melting. EMSP glasses were prepared from AZ-101 simulant and additives $\left(\mathrm{H}_{3} \mathrm{BO}_{3}\right.$, carbonates of $\mathrm{Li}$ and $\mathrm{Na}$, and $\left.\mathrm{SiO}_{2}\right)$ as well as from raw chemicals. For glasses made from raw chemicals, trace quantities of elemental $\mathrm{Ru}$ (in the form of ruthenium nitrosyl nitrate solution) were added drop by drop to $100 \mathrm{~g}$ of $\mathrm{SiO}_{2}$ that was dispersed on a Petri dish. $\mathrm{SiO}_{2}$ cake was dried in oven at $105^{\circ} \mathrm{C}$ for 1 hour, quenched, and hand-mixed in the plastic bag with the rest of the glass batch. Then, the glass batch was milled in an agate mill for $5 \mathrm{~min}$ to ensure homogeneity.

EMSP glasses were produced in Pt-alloy crucibles following a two-step melting process: 1) melting of homogenized glass feeds and 2) melting of glasses from feeds after quenching and grinding. The melting temperature for Ni1.5/A112 and $\mathrm{Fe} 20 / \mathrm{Ni1} .5$ glasses was $1250^{\circ} \mathrm{C}$ and $1300^{\circ} \mathrm{C}$, respectively. The other EMSP glasses were melted at $1200^{\circ} \mathrm{C}$.

(a) GDL-GBM, Rev. 3. 2003. Glass Batching and Melting. Pacific Northwest National Laboratory, Technical Procedure. 



\subsection{Analytical Results for AZ-101 Simulant and Selected EMSP Glasses}

A representative sample of AZ-101 simulant, eight samples of EMSP glasses prepared from AZ-101 simulant, and two samples of EMSP glasses prepared from raw chemicals were sent for analysis of metals to Southwest Research Institute (SWRI) to confirm that their compositions are in good agreement with the defined target compositions. Methods blanks, duplicates, spikes, and reference glass standards (National Institute for Standards and Technology [NIST] SRM 278 Obsidian Rock and NIST SRM 688 Basalt Rock) were run to assess the precision and accuracy of analysis with inductively coupled plasmamass spectrometry (ICP-MS) and inductively coupled plasma-atomic emission spectroscopy (ICP-AES).

\subsection{AZ-101 Simulant}

A dried and ground sample of AZ-101 simulant was converted into liquid using four techniques: 1) $\mathrm{LiBO}_{2} / \mathrm{Li}_{2} \mathrm{~B}_{4} \mathrm{O}_{7}$ fusion to determine $\mathrm{Al}, \mathrm{Ba}, \mathrm{Ca}, \mathrm{Cr}, \mathrm{Co}, \mathrm{Fe}, \mathrm{Mn}, \mathrm{P}, \mathrm{Si}, \mathrm{Na}, \mathrm{Sn}, \mathrm{Ti}$, and $\mathrm{Zr}, 2$ ) $\mathrm{HF} / \mathrm{HNO}_{3}$ digestion to determine $\mathrm{Ru}, 3) \mathrm{HNO}_{3} / \mathrm{HCl} / \mathrm{HF}$ digestion in closed vessel to determine $\mathrm{B}$ and $\mathrm{S}$, and 4) $\mathrm{HNO}_{3} / \mathrm{HClO}_{4} / \mathrm{HF} / \mathrm{HCl}$ digestion in open vessel to determine $\mathrm{Cd}, \mathrm{Cu}, \mathrm{La}, \mathrm{Pb}, \mathrm{Li}, \mathrm{Mg}, \mathrm{Mo}, \mathrm{Ni}, \mathrm{K}, \mathrm{Rh}$, and $\mathrm{Zn}$. Produced solutions were analyzed for $\mathrm{Co}, \mathrm{Rh}, \mathrm{Ru}, \mathrm{Ti}$, and $\mathrm{Zn}$ with ICP-mass spectrometry (MS) and for all other metals with ICP-AES.

Table 4.1 compares the analyzed composition of AZ-101 simulant with the composition data of simulant available in Eibling et al. The analyzed alumina content matched nicely the targeted value. In contrast, the chemical analyses also indicated somewhat lower than tabulated concentrations for some major components such as $\mathrm{B}_{2} \mathrm{O}_{3}, \mathrm{Fe}_{2} \mathrm{O}_{3}, \mathrm{Na}_{2} \mathrm{O}, \mathrm{SiO}_{2}$, and $\mathrm{ZrO}_{2}$. The relatively large relative percent differences (RPDs) can be explained by errors introduced while the simulant was being prepared as well as analysis errors.

\subsection{EMSP Glasses}

Half of the samples from EMSP glasses were prepared from quenched and ground first melts [EMSPXX-(AZ-101)-1M; EMSP-Ni1.5-(Chem.)-1M], and the other half was extracted and ground from the middle section of double crucibles that were heat-treated at $850^{\circ} \mathrm{C}$ for 7 or 8 days [EMSP-XX-(AZ-101)7D or 8D; EMSP-Ni1.5-(Chem.)-7D]. The glass powder was converted into liquid using four techniques: 1) $\mathrm{HF} / \mathrm{HNO}_{3}$ digestion to determine $\left.\mathrm{Ru}, 2\right) \mathrm{HNO}_{3} / \mathrm{HCl} / \mathrm{HF}$ digestion in closed vessel to determine $\mathrm{B}$, 3) $\mathrm{HNO}_{3} / \mathrm{HClO}_{4} / \mathrm{HF} / \mathrm{HCl}$ digestion in open vessel to determine $\mathrm{Cd}, \mathrm{Ca}, \mathrm{Co}, \mathrm{Cu}, \mathrm{Li}, \mathrm{Mg}, \mathrm{K}, \mathrm{Rh}, \mathrm{Na}, \mathrm{S}, \mathrm{Sn}$, and $\mathrm{Zn}$, and 4) $\mathrm{LiBO}_{2} / \mathrm{Li}_{2} \mathrm{~B}_{4} \mathrm{O}_{7}$ fusion to determine Al, Ba, Ce, Cr, Fe, La, Mn, Nd, Ni, P, Si, Ti, and Zr. Produced solutions were analyzed for $\mathrm{Ce}, \mathrm{La}, \mathrm{Nd}, \mathrm{Rh}$, and $\mathrm{Ru}$ with ICP-MS and for all other metals with ICP-AES.

Table 4.2, Table 4.4, Table 4.6 and Table 4.8 compare the analyzed compositions of AZ-101 glasses: baseline glass (EMSP-BL-AZ-101), Cr-rich (EMSP-Cr0.6-AZ-101), Ni-rich (EMSP-Ni1.5-AZ-101), and Fe-rich (EMSP-Fe20-AZ-101) glasses after their first melt with target compositions, respectively. The glass compositions are in good agreement with tabulated values, and concentrations of major components are within the error of the analysis. The lower concentrations of $\mathrm{Fe}_{2} \mathrm{O}_{3}$ are the result of a lower-than-

tabulated concentration of $\mathrm{Fe}_{2} \mathrm{O}_{3}$ in the $\mathrm{HLW}$ simulant. Additional $\mathrm{Ce}_{2} \mathrm{O}_{3}$ and $\mathrm{SnO}_{2}$ come from impurities 
of used chemicals. Table 4.3, Table 4.5, Table 4.7 and Table 4.9 compare the analyzed compositions for the above-mentioned glasses after the first melt and after the double crucible test at $850^{\circ} \mathrm{C}$ for 7 or 8 days [EMSP-Fe20-8D-(AZ-101)]. The concentrations of spinel-forming components $\left(\mathrm{Fe}_{2} \mathrm{O}_{3}, \mathrm{Cr}_{2} \mathrm{O}_{3}\right.$, and $\left.\mathrm{NiO}\right)$ in the glasses after 7 days have decreased because the precipitated spinel crystals have settled. Ni-rich glass was an extreme case. Considerably lower concentrations of $\mathrm{Fe}, \mathrm{Cr}$, and $\mathrm{Ni}$ were obtained for this glass because most of the large crystals from the top and middle of the crucible already settled, leaving nearly crystal-free glass. On the contrary, no change in the concentration of spinel-forming components was observed for Fe-rich glass because of the slow settling rate of less than $10-\mu \mathrm{m}$ crystals. These crystals were entrapped in the glass sample and were included in the analysis. Table 4.10 compares the analyzed composition of Ni-rich glass prepared from chemicals with target compositions. The concentrations of all the components, except for $\mathrm{Rh}_{2} \mathrm{O}_{3}$, were close to tabulated values, indicating a properly prepared glass. Only about $25 \mathrm{wt} \%$ of total the $\mathrm{Rh}_{2} \mathrm{O}_{3}$ was detected in the glass sample. This discrepancy can be explained by a non-uniform distribution of high-density $\mathrm{Rh}_{2} \mathrm{O}_{3}$ particles because of their tendency to form large agglomerates that can accumulate in the different parts of the sample. Table 4.11 compares the glass compositions of EMSP-Ni1.5-(Chem.) after first melt and after 7 days at $850^{\circ} \mathrm{C}$. Their compositions were almost identical, including the concentrations of $\mathrm{NiO}, \mathrm{Fe}_{2} \mathrm{O}_{3}$, and $\mathrm{Cr}_{2} \mathrm{O}_{3}$. 
Table 4.1. Analyzed Composition of AZ-101 Simulant Compared with Target Composition of Simulant from Literature

\begin{tabular}{|c|c|c|c|c|c|c|}
\hline Component & $\begin{array}{c}\text { AZ-101 } \\
\text { (Eibling et al.) }\end{array}$ & $\begin{array}{l}\text { AZ-101 } \\
\text { (Analyzed) }\end{array}$ & Average & SD & RSD, \% & RPD, \% \\
\hline $\mathrm{Al}_{2} \mathrm{O}_{3}$ & 0.2263 & 0.2267 & 0.2265 & 0.0003 & 0.1 & 0.2 \\
\hline $\mathrm{B}_{2} \mathrm{O}_{3}$ & 0.0159 & 0.0012 & 0.0086 & 0.0104 & 121.2 & -92.3 \\
\hline $\mathrm{BaO}$ & 0.0026 & 0.0020 & 0.0023 & 0.0004 & 17.9 & -22.5 \\
\hline $\mathrm{CaO}$ & 0.0158 & 0.0116 & 0.0137 & 0.0030 & 21.7 & -26.6 \\
\hline $\mathrm{CdO}$ & 0.0178 & 0.0185 & 0.0181 & 0.0005 & 2.8 & 4.1 \\
\hline $\mathrm{Cr}_{2} \mathrm{O}_{3}$ & 0.0047 & 0.0038 & 0.0043 & 0.0007 & 16.0 & -20.4 \\
\hline F & 0.0002 & NA & - & - & - & - \\
\hline $\mathrm{Fe}_{2} \mathrm{O}_{3}$ & 0.3999 & 0.3224 & 0.3611 & 0.0548 & 15.2 & -19.4 \\
\hline $\mathrm{K}_{2} \mathrm{O}$ & 0.0095 & 0.0035 & 0.0065 & 0.0042 & 65.4 & -63.2 \\
\hline $\mathrm{MgO}$ & 0.0036 & 0.0026 & 0.0031 & 0.0007 & 22.0 & -26.9 \\
\hline $\mathrm{MnO}$ & 0.0097 & 0.0077 & 0.0087 & 0.0014 & 15.9 & -20.2 \\
\hline $\mathrm{Na}_{2} \mathrm{O}$ & 0.0786 & 0.0663 & 0.0724 & 0.0088 & 12.1 & -15.7 \\
\hline $\mathrm{NiO}$ & 0.0175 & 0.0137 & 0.0156 & 0.0027 & 17.5 & -22.0 \\
\hline $\mathrm{P}_{2} \mathrm{O}_{5}$ & 0.0088 & 0.0088 & 0.0088 & 0.0000 & 0.4 & 0.6 \\
\hline $\mathrm{Rh}_{2} \mathrm{O}_{3}$ & 0.0009 & $2.6 \mathrm{E}-07$ & 0.0005 & 0.0007 & 141.3 & -100.0 \\
\hline $\mathrm{RuO}_{2}$ & 0.0017 & BRL & - & - & - & - \\
\hline $\mathrm{SiO}_{2}$ & 0.0467 & 0.0346 & 0.0406 & 0.0086 & 21.1 & -26.0 \\
\hline $\mathrm{SO}_{3}$ & 0.0023 & 0.0017 & 0.0020 & 0.0004 & 20.5 & -25.4 \\
\hline $\mathrm{TiO}_{2}$ & 0.0008 & 0.0004 & 0.0006 & 0.0003 & 52.5 & -54.2 \\
\hline $\mathrm{ZnO}$ & 0.0006 & 0.0004 & 0.0005 & 0.0001 & 29.3 & -34.3 \\
\hline $\mathrm{ZrO}_{2}$ & 0.1148 & 0.0831 & 0.0989 & 0.0224 & 22.7 & -27.6 \\
\hline $\mathrm{Cl}$ & 0.0006 & NA & - & - & - & - \\
\hline $\mathrm{Ce}_{2} \mathrm{O}_{3}$ & 0.0056 & 0.0067 & 0.0061 & 0.0008 & 13.0 & 20.2 \\
\hline $\mathrm{CoO}$ & 0.0003 & 0.0002 & 0.0002 & 0.0001 & 25.5 & -30.5 \\
\hline $\mathrm{CuO}$ & 0.0011 & 0.0009 & 0.0010 & 0.0001 & 14.3 & -18.3 \\
\hline $\mathrm{La}_{2} \mathrm{O}_{3}$ & 0.0061 & 0.0071 & 0.0066 & 0.0007 & 11.3 & 17.4 \\
\hline $\mathrm{Nd}_{2} \mathrm{O}_{3}$ & 0.0050 & 0.0054 & 0.0052 & 0.0003 & 5.1 & 7.6 \\
\hline $\mathrm{SnO}_{2}$ & 0.0027 & 0.0040 & 0.0034 & 0.0009 & 27.6 & 48.5 \\
\hline LOI & - & 0.2011 & - & - & - & - \\
\hline Total & 1.0000 & 1.0342 & - & - & - & - \\
\hline
\end{tabular}

(a) Does not include $0.0019 \mathrm{PbO}, 0.0001 \mathrm{MoO}_{3}, 0.0045 \mathrm{SrO}, 0.0003 \mathrm{Th}_{2} \mathrm{O}_{3}$, and $2 \mathrm{E}-5 \mathrm{As}_{2} \mathrm{O}_{3}$;

SD: Standard deviation; RSD: Relative standard deviation (SD/Average); RPD: Relative percent difference between analyzed and literature compositions; NA: Not analyzed; BRL: Below reporting limit $(<1.3 \mathrm{E}-07)$; LOI: Loss on ignition at $1100^{\circ} \mathrm{C}$; (-) Empty field. 
Table 4.2. Analyzed Composition of Baseline Glass EMSP-BL-(AZ-101)-1M, After First Melt, Compared with Target Values for Designed BL Glass

\begin{tabular}{|c|c|c|c|c|c|c|}
\hline Component & $\mathrm{BL}$ & BL-1M & Average & SD & RSD, $\%$ & RPD, \% \\
\hline $\mathrm{Al}_{2} \mathrm{O}_{3}$ & 0.0821 & 0.0861 & 0.0841 & 0.0028 & 3.4 & 4.9 \\
\hline $\mathrm{B}_{2} \mathrm{O}_{3}$ & 0.0799 & 0.0757 & 0.0778 & 0.0030 & 3.8 & -5.3 \\
\hline $\mathrm{BaO}$ & 0.0009 & 0.0007 & 0.0008 & 0.0001 & 17.7 & -22.2 \\
\hline $\mathrm{CaO}$ & 0.0057 & 0.0047 & 0.0052 & 0.0007 & 13.6 & -17.5 \\
\hline $\mathrm{CdO}$ & 0.0065 & 0.0071 & 0.0068 & 0.0004 & 6.2 & 9.2 \\
\hline $\mathrm{Cr}_{2} \mathrm{O}_{3}$ & 0.0017 & 0.0014 & 0.0016 & 0.0002 & 13.7 & -17.6 \\
\hline F & 0.0001 & NM & - & - & - & - \\
\hline $\mathrm{Fe}_{2} \mathrm{O}_{3}$ & 0.1451 & 0.1205 & 0.1328 & 0.0174 & 13.1 & -17.0 \\
\hline $\mathrm{K}_{2} \mathrm{O}$ & 0.0034 & 0.0012 & 0.0023 & 0.0016 & 67.6 & -64.7 \\
\hline $\mathrm{Li}_{2} \mathrm{O}$ & 0.0199 & 0.0195 & 0.0197 & 0.0003 & 1.4 & -2.0 \\
\hline $\mathrm{MgO}$ & 0.0013 & 0.0011 & 0.0012 & 0.0001 & 11.8 & -15.4 \\
\hline $\mathrm{MnO}$ & 0.0035 & 0.0030 & 0.0033 & 0.0004 & 10.9 & -14.3 \\
\hline $\mathrm{Na}_{2} \mathrm{O}$ & 0.1866 & 0.1773 & 0.1820 & 0.0066 & 3.6 & -5.0 \\
\hline $\mathrm{NiO}$ & 0.0064 & 0.0055 & 0.0060 & 0.0006 & 10.7 & -14.1 \\
\hline $\mathrm{P}_{2} \mathrm{O}_{5}$ & 0.0032 & 0.0034 & 0.0033 & 0.0001 & 4.3 & 6.2 \\
\hline $\mathrm{Rh}_{2} \mathrm{O}_{3}$ & NA & $1.2 \mathrm{E}-06$ & - & - & - & - \\
\hline $\mathrm{RuO}_{2}$ & NA & $2.2 \mathrm{E}-07$ & - & - & - & - \\
\hline $\mathrm{SiO}_{2}$ & 0.4031 & 0.4033 & 0.4032 & 0.0001 & 0.0 & 0.0 \\
\hline $\mathrm{SO}_{3}$ & 0.0008 & 0.0002 & 0.0005 & 0.0004 & 84.9 & -75.0 \\
\hline $\mathrm{TiO}_{2}$ & 0.0003 & 0.0003 & 0.0003 & 0.0000 & 0.0 & 0.0 \\
\hline $\mathrm{ZnO}$ & 0.0002 & 0.0001 & 0.0002 & 0.0001 & 47.1 & -50.0 \\
\hline $\mathrm{ZrO}_{2}$ & 0.0416 & 0.0346 & 0.0381 & 0.0049 & 13.0 & -16.8 \\
\hline $\mathrm{Cl}$ & 0.0002 & NM & - & - & - & - \\
\hline $\mathrm{Ce}_{2} \mathrm{O}_{3}$ & 0.0020 & 0.0054 & 0.0037 & 0.0024 & 65.0 & 170.0 \\
\hline $\mathrm{CoO}$ & 0.0001 & 0.0001 & 0.0001 & 0.0000 & 0.0 & 0.0 \\
\hline $\mathrm{CuO}$ & 0.0004 & 0.0003 & 0.0004 & 0.0001 & 20.2 & -25.0 \\
\hline $\mathrm{La}_{2} \mathrm{O}_{3}$ & 0.0022 & 0.0029 & 0.0026 & 0.0005 & 19.4 & 31.8 \\
\hline $\mathrm{Nd}_{2} \mathrm{O}_{3}$ & 0.0018 & 0.0021 & 0.0020 & 0.0002 & 10.9 & 16.7 \\
\hline $\mathrm{SnO}_{2}$ & 0.0010 & 0.0019 & 0.0015 & 0.0006 & 43.9 & 90.0 \\
\hline LOI & - & 0.0209 & - & - & - & - \\
\hline Total & 1.0000 & 0.9793 & - & - & - & - \\
\hline
\end{tabular}

SD: Standard deviation; RSD: Relative standard deviation (SD/Average); RPD: Relative percent difference between analyzed and designed compositions; NM: Not measured; (-) Empty field; NA: Not analyzed; LOI: Loss on ignition at $1000^{\circ} \mathrm{C}$. 
Table 4.3. Analyzed Composition of Baseline Glass EMSP-BL-(AZ-101)-1M, after First Melt, Compared with Analyzed Composition of Baseline Glass EMSP-BL-(AZ-101)-7D, after 7 days at $850^{\circ} \mathrm{C}$ (Double Crucible Test)

\begin{tabular}{|c|c|c|c|c|c|c|}
\hline Component & BL-1M & BL-7D & Average & SD & RSD, \% & RPD, \% \\
\hline $\mathrm{Al}_{2} \mathrm{O}_{3}$ & 0.0861 & 0.0865 & 0.0863 & 0.0003 & 0.3 & 0.5 \\
\hline $\mathrm{B}_{2} \mathrm{O}_{3}$ & 0.0757 & 0.0860 & 0.0809 & 0.0073 & 9.0 & 13.6 \\
\hline $\mathrm{BaO}$ & 0.0007 & 0.0007 & 0.0007 & 0.0000 & 0.0 & 0.0 \\
\hline $\mathrm{CaO}$ & 0.0047 & 0.0047 & 0.0047 & 0.0000 & 0.0 & 0.0 \\
\hline $\mathrm{CdO}$ & 0.0071 & 0.0070 & 0.0071 & 0.0001 & 1.0 & -1.4 \\
\hline $\mathrm{Cr}_{2} \mathrm{O}_{3}$ & 0.0014 & 0.0013 & 0.0014 & 0.0001 & 5.2 & -7.1 \\
\hline $\mathrm{Fe}_{2} \mathrm{O}_{3}$ & 0.1205 & 0.1168 & 0.1187 & 0.0026 & 2.2 & -3.1 \\
\hline $\mathrm{K}_{2} \mathrm{O}$ & 0.0012 & 0.0016 & 0.0014 & 0.0003 & 20.2 & 33.3 \\
\hline $\mathrm{Li}_{2} \mathrm{O}$ & 0.0195 & 0.0194 & 0.0195 & 0.0001 & 0.4 & -0.5 \\
\hline $\mathrm{MgO}$ & 0.0011 & 0.0011 & 0.0011 & 0.0000 & 0.0 & 0.0 \\
\hline $\mathrm{MnO}$ & 0.0030 & 0.0030 & 0.0030 & 0.0000 & 0.0 & 0.0 \\
\hline $\mathrm{Na}_{2} \mathrm{O}$ & 0.1773 & 0.1766 & 0.1770 & 0.0005 & 0.3 & -0.4 \\
\hline $\mathrm{NiO}$ & 0.0055 & 0.0042 & 0.0049 & 0.0009 & 19.0 & -23.6 \\
\hline $\mathrm{P}_{2} \mathrm{O}_{5}$ & 0.0034 & 0.0034 & 0.0034 & 0.0000 & 0.0 & 0.0 \\
\hline $\mathrm{Rh}_{2} \mathrm{O}_{3}$ & $1.2 \mathrm{E}-06$ & $8.1 \mathrm{E}-07$ & $1.0 \mathrm{E}-06$ & $2.8 \mathrm{E}-07$ & 27.4 & -32.5 \\
\hline $\mathrm{RuO}_{2}$ & $2.2 \mathrm{E}-07$ & $2.2 \mathrm{E}-07$ & 2.2E-07 & $0.0 \mathrm{E}+00$ & 0.0 & 0.0 \\
\hline $\mathrm{SiO}_{2}$ & 0.4033 & 0.4065 & 0.4049 & 0.0023 & 0.6 & 0.8 \\
\hline $\mathrm{SO}_{3}$ & 0.0002 & 0.0004 & 0.0003 & 0.0001 & 47.1 & 100.0 \\
\hline $\mathrm{TiO}_{2}$ & 0.0003 & 0.0002 & 0.0003 & 0.0001 & 28.3 & -33.3 \\
\hline $\mathrm{ZnO}$ & 0.0001 & 0.0001 & 0.0001 & 0.0000 & 0.0 & 0.0 \\
\hline $\mathrm{ZrO}_{2}$ & 0.0346 & 0.0350 & 0.0348 & 0.0003 & 0.8 & 1.2 \\
\hline $\mathrm{Ce}_{2} \mathrm{O}_{3}$ & 0.0054 & 0.0053 & 0.0054 & 0.0001 & 1.3 & -1.9 \\
\hline $\mathrm{CoO}$ & 0.0001 & 0.0001 & 0.0001 & 0.0000 & 0.0 & 0.0 \\
\hline $\mathrm{CuO}$ & 0.0003 & 0.0003 & 0.0003 & 0.0000 & 0.0 & 0.0 \\
\hline $\mathrm{La}_{2} \mathrm{O}_{3}$ & 0.0029 & 0.0029 & 0.0029 & 0.0000 & 0.0 & 0.0 \\
\hline $\mathrm{Nd}_{2} \mathrm{O}_{3}$ & 0.0021 & 0.0021 & 0.0021 & 0.0000 & 0.0 & 0.0 \\
\hline $\mathrm{SnO}_{2}$ & 0.0019 & 0.0020 & 0.0020 & 0.0001 & 3.6 & 5.3 \\
\hline LOI & 0.0209 & 0.0256 & 0.0233 & 0.0033 & 14.3 & 22.5 \\
\hline Total & 0.9793 & 0.9928 & 0.9861 & 0.0095 & 1.0 & 1.4 \\
\hline
\end{tabular}


Table 4.4. Analyzed Composition of EMSP-Cr0.6-(AZ-101)-1M) Glass after First Melt Compared with Target Values for Designed Cr0.6 Glass

\begin{tabular}{|c|c|c|c|c|c|c|}
\hline Component & Cr0.6 & Cr0.6-1M & Average & SD & RSD, \% & RPD, $\%$ \\
\hline $\mathrm{Al}_{2} \mathrm{O}_{3}$ & 0.0817 & 0.0875 & 0.0846 & 0.0041 & 4.8 & 7.1 \\
\hline $\mathrm{B}_{2} \mathrm{O}_{3}$ & 0.0796 & 0.0728 & 0.0762 & 0.0048 & 6.3 & -8.5 \\
\hline $\mathrm{BaO}$ & 0.0009 & 0.0007 & 0.0008 & 0.0001 & 17.7 & -22.2 \\
\hline $\mathrm{CaO}$ & 0.0057 & 0.0045 & 0.0051 & 0.0008 & 16.6 & -21.1 \\
\hline $\mathrm{CdO}$ & 0.0065 & 0.0070 & 0.0068 & 0.0004 & 5.2 & 7.7 \\
\hline $\mathrm{Cr}_{2} \mathrm{O}_{3}$ & 0.0060 & 0.0058 & 0.0059 & 0.0001 & 2.4 & -3.3 \\
\hline F & 0.0001 & NM & - & - & - & - \\
\hline $\mathrm{Fe}_{2} \mathrm{O}_{3}$ & 0.1445 & 0.1192 & 0.1319 & 0.0179 & 13.6 & -17.5 \\
\hline $\mathrm{K}_{2} \mathrm{O}$ & 0.0034 & 0.0018 & 0.0026 & 0.0011 & 43.5 & -47.1 \\
\hline $\mathrm{Li}_{2} \mathrm{O}$ & 0.0198 & 0.0195 & 0.0197 & 0.0002 & 1.1 & -1.5 \\
\hline $\mathrm{MgO}$ & 0.0013 & 0.0012 & 0.0013 & 0.0001 & 5.7 & -7.7 \\
\hline $\mathrm{MnO}$ & 0.0035 & 0.0030 & 0.0033 & 0.0004 & 10.9 & -14.3 \\
\hline $\mathrm{Na}_{2} \mathrm{O}$ & 0.1858 & 0.1766 & 0.1812 & 0.0065 & 3.6 & -5.0 \\
\hline $\mathrm{NiO}$ & 0.0064 & 0.0053 & 0.0059 & 0.0008 & 13.3 & -17.2 \\
\hline $\mathrm{P}_{2} \mathrm{O}_{5}$ & 0.0032 & 0.0034 & 0.0033 & 0.0001 & 4.3 & 6.2 \\
\hline $\mathrm{Rh}_{2} \mathrm{O}_{3}$ & NA & 7.6E-07 & - & - & - & - \\
\hline $\mathrm{RuO}_{2}$ & NA & BRL & - & - & - & - \\
\hline $\mathrm{SiO}_{2}$ & 0.4014 & 0.4065 & 0.4040 & 0.0036 & 0.9 & 1.3 \\
\hline $\mathrm{SO}_{3}$ & 0.0008 & 0.0003 & 0.0006 & 0.0004 & 64.3 & -62.5 \\
\hline $\mathrm{TiO}_{2}$ & 0.0003 & 0.0003 & 0.0003 & 0.0000 & 0.0 & 0.0 \\
\hline $\mathrm{ZnO}$ & 0.0002 & 0.0001 & 0.0002 & 0.0001 & 47.1 & -50.0 \\
\hline $\mathrm{ZrO}_{2}$ & 0.0414 & 0.0346 & 0.0380 & 0.0048 & 12.7 & -16.4 \\
\hline $\mathrm{Cl}$ & 0.0002 & NM & - & - & - & - \\
\hline $\mathrm{Ce}_{2} \mathrm{O}_{3}$ & 0.0020 & 0.0054 & 0.0037 & 0.0024 & 65.0 & 170.0 \\
\hline $\mathrm{CoO}$ & 0.0001 & 0.0001 & 0.0001 & 0.0000 & 0.0 & 0.0 \\
\hline $\mathrm{CuO}$ & 0.0004 & 0.0003 & 0.0004 & 0.0001 & 20.2 & -25.0 \\
\hline $\mathrm{La}_{2} \mathrm{O}_{3}$ & 0.0022 & 0.0029 & 0.0026 & 0.0005 & 19.4 & 31.8 \\
\hline $\mathrm{Nd}_{2} \mathrm{O}_{3}$ & 0.0018 & 0.0021 & 0.0020 & 0.0002 & 10.9 & 16.7 \\
\hline $\mathrm{SnO}_{2}$ & 0.0010 & 0.0020 & 0.0015 & 0.0007 & 47.1 & 100.0 \\
\hline LOI & - & 0.0213 & - & - & - & - \\
\hline Total & 1.0000 & 0.9842 & - & - & - & - \\
\hline
\end{tabular}

SD: Standard deviation; RSD: Relative standard deviation (SD/Average); RPD: Relative percent difference between analyzed and designed compositions; NM: Not measured; (-) Empty field; NA: Not analyzed; BRL: Below reporting limit; LOI: Loss on ignition at $1000^{\circ} \mathrm{C}$. 
Table 4.5. Analyzed Composition of EMSP-Cr0.6-(AZ-101)-1M Glass after First Melt Compared with Analyzed Composition of EMSP-Cr0.6 (AZ-101)-7D Glass after 7 days at $850^{\circ} \mathrm{C}$ (Double Crucible Test)

\begin{tabular}{|c|c|c|c|c|c|c|}
\hline Component & $\mathrm{Cr} 0.6-1 \mathrm{M}$ & Cr0.6-7D & Average & SD & RSD, \% & RPD, \% \\
\hline $\mathrm{Al}_{2} \mathrm{O}_{3}$ & 0.0875 & 0.0862 & 0.0869 & 0.0009 & 1.1 & -1.5 \\
\hline $\mathrm{B}_{2} \mathrm{O}_{3}$ & 0.0728 & 0.0811 & 0.0770 & 0.0059 & 7.6 & 11.4 \\
\hline $\mathrm{BaO}$ & 0.0007 & 0.0007 & 0.0007 & 0.0000 & 0.0 & 0.0 \\
\hline $\mathrm{CaO}$ & 0.0045 & 0.0046 & 0.0046 & 0.0001 & 1.6 & 2.2 \\
\hline $\mathrm{CdO}$ & 0.007 & 0.0071 & 0.0071 & 0.0001 & 1.0 & 1.4 \\
\hline $\mathrm{Cr}_{2} \mathrm{O}_{3}$ & 0.0058 & 0.0059 & 0.0059 & 0.0001 & 1.2 & 1.7 \\
\hline $\mathrm{Fe}_{2} \mathrm{O}_{3}$ & 0.1192 & 0.1221 & 0.1207 & 0.0021 & 1.7 & 2.4 \\
\hline $\mathrm{K}_{2} \mathrm{O}$ & 0.0018 & 0.0017 & 0.0018 & 0.0001 & 4.0 & -5.6 \\
\hline $\mathrm{Li}_{2} \mathrm{O}$ & 0.0195 & 0.0194 & 0.0195 & 0.0001 & 0.4 & -0.5 \\
\hline $\mathrm{MgO}$ & 0.0012 & 0.0011 & 0.0012 & 0.0001 & 6.1 & -8.3 \\
\hline $\mathrm{MnO}$ & 0.003 & 0.0030 & 0.0030 & 0.0000 & 0.0 & 0.0 \\
\hline $\mathrm{Na}_{2} \mathrm{O}$ & 0.1766 & 0.1766 & 0.1766 & 0.0000 & 0.0 & 0.0 \\
\hline $\mathrm{NiO}$ & 0.0053 & 0.0054 & 0.0054 & 0.0001 & 1.3 & 1.9 \\
\hline $\mathrm{P}_{2} \mathrm{O}_{5}$ & 0.0034 & 0.0032 & 0.0033 & 0.0001 & 4.3 & -5.9 \\
\hline $\mathrm{Rh}_{2} \mathrm{O}_{3}$ & 7.6E-07 & 7.7E-07 & 7.65E-07 & 7.1E-09 & 0.9 & 1.3 \\
\hline $\mathrm{RuO}_{2}$ & BRL & BRL & - & - & - & - \\
\hline $\mathrm{SiO}_{2}$ & 0.4065 & 0.4065 & 0.4065 & 0.0000 & 0.0 & 0.0 \\
\hline $\mathrm{SO}_{3}$ & 0.0003 & 0.0003 & 0.0003 & 0.0000 & 0.0 & 0.0 \\
\hline $\mathrm{TiO}_{2}$ & 0.0003 & 0.0002 & 0.0003 & 0.0001 & 28.3 & -33.3 \\
\hline $\mathrm{ZnO}$ & 0.0001 & 0.0001 & 0.0001 & 0.0000 & 0.0 & 0.0 \\
\hline $\mathrm{ZrO}_{2}$ & 0.0346 & 0.0347 & 0.0347 & 0.0001 & 0.2 & 0.3 \\
\hline $\mathrm{Ce}_{2} \mathrm{O}_{3}$ & 0.0054 & 0.0054 & 0.0054 & 0.0000 & 0.0 & 0.0 \\
\hline $\mathrm{CoO}$ & 0.0001 & 0.0001 & 0.0001 & 0.0000 & 0.0 & 0.0 \\
\hline $\mathrm{CuO}$ & 0.0003 & 0.0003 & 0.0003 & 0.0000 & 0.0 & 0.0 \\
\hline $\mathrm{La}_{2} \mathrm{O}_{3}$ & 0.0029 & 0.0029 & 0.0029 & 0.0000 & 0.0 & 0.0 \\
\hline $\mathrm{Nd}_{2} \mathrm{O}_{3}$ & 0.0021 & 0.0021 & 0.0021 & 0.0000 & 0.0 & 0.0 \\
\hline $\mathrm{SnO}_{2}$ & 0.0020 & 0.0020 & 0.0020 & 0.0000 & 0.0 & 0.0 \\
\hline LOI & 0.0213 & 0.0209 & 0.0211 & 0.0003 & 1.3 & -1.9 \\
\hline Total & 0.9842 & 0.9936 & 0.9889 & 0.0066 & 0.7 & 1.0 \\
\hline
\end{tabular}

SD: Standard deviation; RSD: Relative standard deviation (SD/Average); RPD: Relative percent difference between analyzed compositions; BRL: Below reporting limit; (-) Empty field; LOI: Loss on ignition at $1000^{\circ} \mathrm{C}$. 
Table 4.6. Analyzed Composition of EMSP-Ni1.5-1M Glass after First Melt Compared with Target Values for Designed Ni1.5 Glass

\begin{tabular}{|c|c|c|c|c|c|c|}
\hline Component & Ni1.5 & Ni1.5-1M & Average & SD & RSD, \% & RPD, \% \\
\hline $\mathrm{Al}_{2} \mathrm{O}_{3}$ & 0.0814 & 0.0899 & 0.0857 & 0.0060 & 7.0 & 10.4 \\
\hline $\mathrm{B}_{2} \mathrm{O}_{3}$ & 0.0792 & 0.0737 & 0.0765 & 0.0039 & 5.1 & -6.9 \\
\hline $\mathrm{BaO}$ & 0.0009 & 0.0007 & 0.0008 & 0.0001 & 17.7 & -22.2 \\
\hline $\mathrm{CaO}$ & 0.0057 & 0.0045 & 0.0051 & 0.0008 & 16.6 & -21.1 \\
\hline $\mathrm{CdO}$ & 0.0064 & 0.0069 & 0.0067 & 0.0004 & 5.3 & 7.8 \\
\hline $\mathrm{Cr}_{2} \mathrm{O}_{3}$ & 0.0017 & 0.0014 & 0.0016 & 0.0002 & 13.7 & -17.6 \\
\hline F & 0.0001 & NM & - & - & - & - \\
\hline $\mathrm{Fe}_{2} \mathrm{O}_{3}$ & 0.1438 & 0.1228 & 0.1333 & 0.0148 & 11.1 & -14.6 \\
\hline $\mathrm{K}_{2} \mathrm{O}$ & 0.0034 & 0.0020 & 0.0027 & 0.0010 & 36.7 & -41.2 \\
\hline $\mathrm{Li}_{2} \mathrm{O}$ & 0.0197 & 0.0192 & 0.0195 & 0.0004 & 1.8 & -2.5 \\
\hline $\mathrm{MgO}$ & 0.0013 & 0.0011 & 0.0012 & 0.0001 & 11.8 & -15.4 \\
\hline $\mathrm{MnO}$ & 0.0035 & 0.0031 & 0.0033 & 0.0003 & 8.6 & -11.4 \\
\hline $\mathrm{Na}_{2} \mathrm{O}$ & 0.1850 & 0.1752 & 0.1801 & 0.0069 & 3.8 & -5.3 \\
\hline $\mathrm{NiO}$ & 0.0150 & 0.0146 & 0.0148 & 0.0003 & 1.9 & -2.7 \\
\hline $\mathrm{P}_{2} \mathrm{O}_{5}$ & 0.0032 & 0.0034 & 0.0033 & 0.0001 & 4.3 & 6.2 \\
\hline $\mathrm{Rh}_{2} \mathrm{O}_{3}$ & NA & $1.0 \mathrm{E}-06$ & - & - & - & - \\
\hline $\mathrm{RuO}_{2}$ & NA & $2.0 \mathrm{E}-07$ & - & - & - & - \\
\hline $\mathrm{SiO}_{2}$ & 0.3996 & 0.3915 & 0.3956 & 0.0057 & 1.4 & -2.0 \\
\hline $\mathrm{SO}_{3}$ & 0.0008 & 0.0008 & 0.0008 & 0.0000 & 0.0 & 0.0 \\
\hline $\mathrm{TiO}_{2}$ & 0.0003 & 0.0003 & 0.0003 & 0.0000 & 0.0 & 0.0 \\
\hline $\mathrm{ZnO}$ & 0.0002 & 0.0001 & 0.0002 & 0.0001 & 47.1 & -50.0 \\
\hline $\mathrm{ZrO}_{2}$ & 0.0412 & 0.0334 & 0.0373 & 0.0055 & 14.8 & -18.9 \\
\hline $\mathrm{Cl}$ & 0.0002 & NM & - & - & - & - \\
\hline $\mathrm{Ce}_{2} \mathrm{O}_{3}$ & 0.0020 & 0.0052 & 0.0036 & 0.0023 & 62.9 & 160.0 \\
\hline $\mathrm{CoO}$ & 0.0001 & 0.0001 & 0.0001 & 0.0000 & 0.0 & 0.0 \\
\hline $\mathrm{CuO}$ & 0.0004 & 0.0003 & 0.0004 & 0.0001 & 20.2 & -25.0 \\
\hline $\mathrm{La}_{2} \mathrm{O}_{3}$ & 0.0022 & 0.0028 & 0.0025 & 0.0004 & 17.0 & 27.3 \\
\hline $\mathrm{Nd}_{2} \mathrm{O}_{3}$ & 0.0018 & 0.0020 & 0.0019 & 0.0001 & 7.4 & 11.1 \\
\hline $\mathrm{SnO}_{2}$ & 0.0010 & 0.0019 & 0.0015 & 0.0006 & 43.9 & 90.0 \\
\hline LOI & - & 0.0252 & - & - & - & - \\
\hline Total & 1.0000 & 0.9821 & - & - & - & - \\
\hline
\end{tabular}

SD: Standard deviation; RSD: Relative standard deviation (SD/Average); RPD: Relative percent difference between analyzed and designed compositions; NM: Not measured; (-) Empty field; NA: Not analyzed; LOI: Loss on ignition at $1000^{\circ} \mathrm{C}$. 
Table 4.7. Analyzed Composition of EMSP-Ni1.5-1M Glass after First Melt Compared with Analyzed Composition of EMSP-Ni1.5-7D Glass after 7 Days at $850^{\circ} \mathrm{C}$ (Double Crucible Test)

\begin{tabular}{|c|c|c|c|c|c|c|}
\hline Component & Ni1.5-1M & Ni1.5-7D & Average & SD & RSD, \% & RPD, \% \\
\hline $\mathrm{Al}_{2} \mathrm{O}_{3}$ & 0.0899 & 0.0886 & 0.0893 & 0.0009 & 1.0 & -1.4 \\
\hline $\mathrm{B}_{2} \mathrm{O}_{3}$ & 0.0737 & 0.0744 & 0.0741 & 0.0005 & 0.7 & 0.9 \\
\hline $\mathrm{BaO}$ & 0.0007 & 0.0007 & 0.0007 & 0.0000 & 0.0 & 0.0 \\
\hline $\mathrm{CaO}$ & 0.0045 & 0.0048 & 0.0047 & 0.0002 & 4.6 & 6.7 \\
\hline $\mathrm{CdO}$ & 0.0069 & 0.0070 & 0.0070 & 0.0001 & 1.0 & 1.4 \\
\hline $\mathrm{Cr}_{2} \mathrm{O}_{3}$ & 0.0014 & 0.0010 & 0.0012 & 0.0003 & 23.6 & -28.6 \\
\hline $\mathrm{Fe}_{2} \mathrm{O}_{3}$ & 0.1228 & 0.1112 & 0.1170 & 0.0082 & 7.0 & -9.4 \\
\hline $\mathrm{K}_{2} \mathrm{O}$ & 0.0020 & 0.0018 & 0.0019 & 0.0001 & 7.4 & -10.0 \\
\hline $\mathrm{Li}_{2} \mathrm{O}$ & 0.0192 & 0.0196 & 0.0194 & 0.0003 & 1.5 & 2.1 \\
\hline $\mathrm{MgO}$ & 0.0011 & 0.0011 & 0.0011 & 0.0000 & 0.0 & 0.0 \\
\hline $\mathrm{MnO}$ & 0.0031 & 0.0030 & 0.0031 & 0.0001 & 2.3 & -3.2 \\
\hline $\mathrm{Na}_{2} \mathrm{O}$ & 0.1752 & 0.1793 & 0.1773 & 0.0029 & 1.6 & 2.3 \\
\hline $\mathrm{NiO}$ & 0.0146 & 0.0076 & 0.0111 & 0.0049 & 44.6 & -47.9 \\
\hline $\mathrm{P}_{2} \mathrm{O}_{5}$ & 0.0034 & 0.0033 & 0.0034 & 0.0001 & 2.1 & -2.9 \\
\hline $\mathrm{Rh}_{2} \mathrm{O}_{3}$ & $1.0 \mathrm{E}-06$ & $8.7 \mathrm{E}-07$ & $9.4 \mathrm{E}-07$ & $9.2 \mathrm{E}-08$ & 9.8 & -13.0 \\
\hline $\mathrm{RuO}_{2}$ & $2.0 \mathrm{E}-07$ & $2.0 \mathrm{E}-07$ & $2.0 \mathrm{E}-07$ & $0.0 \mathrm{E}+00$ & 0.0 & 0.0 \\
\hline $\mathrm{SiO}_{2}$ & 0.3915 & 0.4172 & 0.4044 & 0.0182 & 4.5 & 6.6 \\
\hline $\mathrm{SO}_{3}$ & 0.0008 & 0.0008 & 0.0008 & 0.0000 & 0.0 & 0.0 \\
\hline $\mathrm{TiO}_{2}$ & 0.0003 & 0.0002 & 0.0003 & 0.0001 & 28.3 & -33.3 \\
\hline $\mathrm{ZnO}$ & 0.0001 & 0.0001 & 0.0001 & 0.0000 & 0.0 & 0.0 \\
\hline $\mathrm{ZrO}_{2}$ & 0.0334 & 0.0350 & 0.0342 & 0.0011 & 3.3 & 4.8 \\
\hline $\mathrm{Ce}_{2} \mathrm{O}_{3}$ & 0.0052 & 0.0055 & 0.0054 & 0.0002 & 4.0 & 5.8 \\
\hline $\mathrm{CoO}$ & 0.0001 & 0.0001 & 0.0001 & 0.0000 & 0.0 & 0.0 \\
\hline $\mathrm{CuO}$ & 0.0003 & 0.0003 & 0.0003 & 0.0000 & 0.0 & 0.0 \\
\hline $\mathrm{La}_{2} \mathrm{O}_{3}$ & 0.0028 & 0.0029 & 0.0029 & 0.0001 & 2.5 & 3.6 \\
\hline $\mathrm{Nd}_{2} \mathrm{O}_{3}$ & 0.0020 & 0.0021 & 0.0021 & 0.0001 & 3.4 & 5.0 \\
\hline $\mathrm{SnO}_{2}$ & 0.0019 & 0.0019 & 0.0019 & 0.0000 & 0.0 & 0.0 \\
\hline LOI & 0.0252 & 0.0245 & 0.0249 & 0.0005 & 2.0 & -2.8 \\
\hline Total & 0.9821 & 0.9940 & 0.9881 & 0.0084 & 0.9 & 1.2 \\
\hline
\end{tabular}

SD: Standard deviation; RSD: Relative standard deviation (SD/Average); RPD: Relative percent difference between analyzed compositions; BRL: Below reporting limit; LOI: Loss on ignition at $1000^{\circ} \mathrm{C}$. 
Table 4.8. Analyzed Composition of EMSP-Fe20-1M Glass after First Melt Compared with Target Values for Designed Fe20 Glass

\begin{tabular}{|c|c|c|c|c|c|c|}
\hline Component & $\mathrm{Fe} 20$ & $\mathrm{Fe} 20-1 \mathrm{M}$ & Average & SD & RSD, \% & RPD, \% \\
\hline $\mathrm{Al}_{2} \mathrm{O}_{3}$ & 0.0768 & 0.0780 & 0.0774 & 0.0008 & 1.1 & 1.5 \\
\hline $\mathrm{B}_{2} \mathrm{O}_{3}$ & 0.0748 & 0.0708 & 0.0728 & 0.0028 & 3.9 & -5.3 \\
\hline $\mathrm{BaO}$ & 0.0008 & 0.0007 & 0.0008 & 0.0001 & 13.0 & -16.9 \\
\hline $\mathrm{CaO}$ & 0.0053 & 0.0046 & 0.0050 & 0.0005 & 10.4 & -13.8 \\
\hline $\mathrm{CdO}$ & 0.0061 & 0.0067 & 0.0064 & 0.0004 & 6.8 & 10.2 \\
\hline $\mathrm{Cr}_{2} \mathrm{O}_{3}$ & 0.0016 & 0.0014 & 0.0015 & 0.0001 & 9.0 & -12.0 \\
\hline F & 0.0001 & $\mathrm{NM}$ & - & - & - & - \\
\hline $\mathrm{Fe}_{2} \mathrm{O}_{3}$ & 0.2000 & 0.1801 & 0.1901 & 0.0141 & 7.4 & -10.0 \\
\hline $\mathrm{K}_{2} \mathrm{O}$ & 0.0032 & 0.0014 & 0.0023 & 0.0013 & 55.0 & -56.0 \\
\hline $\mathrm{Li}_{2} \mathrm{O}$ & 0.0186 & 0.0180 & 0.0183 & 0.0004 & 2.4 & -3.3 \\
\hline $\mathrm{MgO}$ & 0.0012 & 0.0010 & 0.0011 & 0.0002 & 13.8 & -17.8 \\
\hline $\mathrm{MnO}$ & 0.0033 & 0.0028 & 0.0030 & 0.0003 & 11.1 & -14.5 \\
\hline $\mathrm{Na}_{2} \mathrm{O}$ & 0.1746 & 0.1645 & 0.1696 & 0.0072 & 4.2 & -5.8 \\
\hline $\mathrm{NiO}$ & 0.0060 & 0.0052 & 0.0056 & 0.0006 & 10.0 & -13.2 \\
\hline $\mathrm{P}_{2} \mathrm{O}_{5}$ & 0.0030 & 0.0032 & 0.0031 & 0.0001 & 4.7 & 6.9 \\
\hline $\mathrm{Rh}_{2} \mathrm{O}_{3}$ & NA & $2.00 \mathrm{E}-06$ & - & - & - & - \\
\hline $\mathrm{RuO}_{2}$ & NA & BRL & - & - & - & - \\
\hline $\mathrm{SiO}_{2}$ & 0.3772 & 0.3808 & 0.3790 & 0.0025 & 0.7 & 1.0 \\
\hline $\mathrm{SO}_{3}$ & 0.0007 & 0.0002 & 0.0005 & 0.0004 & 81.8 & -73.3 \\
\hline $\mathrm{TiO}_{2}$ & 0.0003 & 0.0002 & 0.0002 & 0.0001 & 23.8 & -28.8 \\
\hline $\mathrm{ZnO}$ & 0.0002 & 0.0001 & 0.0001 & 0.0001 & 42.9 & -46.6 \\
\hline $\mathrm{ZrO}_{2}$ & 0.0389 & 0.0330 & 0.0360 & 0.0042 & 11.7 & -15.2 \\
\hline $\mathrm{Cl}$ & 0.0002 & $\mathrm{NM}$ & - & - & - & - \\
\hline $\mathrm{Ce}_{2} \mathrm{O}_{3}$ & 0.0019 & 0.0051 & 0.0035 & 0.0023 & 65.5 & 172.5 \\
\hline $\mathrm{CoO}$ & 0.0001 & 0.0001 & 0.0001 & 0.0000 & 4.7 & 6.9 \\
\hline $\mathrm{CuO}$ & 0.0004 & 0.0003 & 0.0003 & 0.0001 & 15.6 & -19.9 \\
\hline $\mathrm{La}_{2} \mathrm{O}_{3}$ & 0.0021 & 0.0027 & 0.0024 & 0.0005 & 19.1 & 31.1 \\
\hline $\mathrm{Nd}_{2} \mathrm{O}_{3}$ & 0.0017 & 0.0020 & 0.0018 & 0.0002 & 12.1 & 18.7 \\
\hline $\mathrm{SnO}_{2}$ & 0.0009 & 0.0019 & 0.0014 & 0.0007 & 48.1 & 103.0 \\
\hline LOI & - & 0.0188 & - & - & - & - \\
\hline Total & 1.0000 & 0.9836 & - & - & - & - \\
\hline
\end{tabular}

SD: Standard deviation; RSD: Relative standard deviation (SD/Average); RPD: Relative percent difference between analyzed and designed compositions; NM: Not measured; (-) Empty field; NA: Not analyzed; LOI: Loss on ignition at $1000^{\circ} \mathrm{C}$. 
Table 4.9. Analyzed Composition of EMSP-Fe20-1M Glass after First Melt Compared with Analyzed Composition of EMSP-Fe20-8D Glass after 8 Days at $850^{\circ} \mathrm{C}$ (Double Crucible Test)

\begin{tabular}{|c|c|c|c|c|c|c|}
\hline Component & Fe20-1M & Fe20-8D & Average & $\mathrm{SD}$ & $\mathrm{RSD}, \%$ & RPD, $\%$ \\
\hline $\mathrm{Al}_{2} \mathrm{O}_{3}$ & 0.0780 & 0.0812 & 0.0796 & 0.0023 & 2.8 & 4.1 \\
\hline $\mathrm{B}_{2} \mathrm{O}_{3}$ & 0.0708 & 0.0699 & 0.0704 & 0.0006 & 0.9 & -1.3 \\
\hline $\mathrm{BaO}$ & 0.0007 & 0.0007 & 0.0007 & 0.0000 & 0.0 & 0.0 \\
\hline $\mathrm{CaO}$ & 0.0046 & 0.0043 & 0.0045 & 0.0002 & 4.8 & -6.5 \\
\hline $\mathrm{CdO}$ & 0.0067 & 0.0066 & 0.0067 & 0.0001 & 1.1 & -1.5 \\
\hline $\mathrm{Cr}_{2} \mathrm{O}_{3}$ & 0.0014 & 0.0014 & 0.0014 & 0.0000 & 0.0 & 0.0 \\
\hline $\mathrm{Fe}_{2} \mathrm{O}_{3}$ & 0.1801 & 0.1787 & 0.1794 & 0.0010 & 0.6 & -0.8 \\
\hline $\mathrm{K}_{2} \mathrm{O}$ & 0.0014 & 0.0014 & 0.0014 & 0.0000 & 0.0 & 0.0 \\
\hline $\mathrm{Li}_{2} \mathrm{O}$ & 0.0180 & 0.0180 & 0.0180 & 0.0000 & 0.0 & 0.0 \\
\hline $\mathrm{MgO}$ & 0.0010 & 0.0010 & 0.0010 & 0.0000 & 0.0 & 0.0 \\
\hline $\mathrm{MnO}$ & 0.0028 & 0.0028 & 0.0028 & 0.0000 & 0.0 & 0.0 \\
\hline $\mathrm{Na}_{2} \mathrm{O}$ & 0.1645 & 0.1645 & 0.1645 & 0.0000 & 0.0 & 0.0 \\
\hline $\mathrm{NiO}$ & 0.0052 & 0.0051 & 0.0052 & 0.0001 & 1.4 & -1.9 \\
\hline $\mathrm{P}_{2} \mathrm{O}_{5}$ & 0.0032 & 0.0035 & 0.0034 & 0.0002 & 6.3 & 9.4 \\
\hline $\mathrm{Rh}_{2} \mathrm{O}_{3}$ & $2.0 \mathrm{E}-06$ & $2.20 \mathrm{E}-06$ & $2.1 \mathrm{E}-06$ & $1.4 \mathrm{E}-07$ & 6.7 & 10.0 \\
\hline $\mathrm{RuO}_{2}$ & BRL & $2.20 \mathrm{E}-07$ & - & - & - & - \\
\hline $\mathrm{SiO}_{2}$ & 0.3808 & 0.3829 & 0.3819 & 0.0015 & 0.4 & 0.6 \\
\hline $\mathrm{SO}_{3}$ & 0.0002 & 0.0008 & 0.0005 & 0.0004 & 84.9 & 300.0 \\
\hline $\mathrm{TiO}_{2}$ & 0.0002 & 0.0002 & 0.0002 & 0.0000 & 0.0 & 0.0 \\
\hline $\mathrm{ZnO}$ & 0.0001 & 0.0001 & 0.0001 & 0.0000 & 0.0 & 0.0 \\
\hline $\mathrm{ZrO}_{2}$ & 0.0330 & 0.0320 & 0.0325 & 0.0007 & 2.2 & -3.0 \\
\hline $\mathrm{Ce}_{2} \mathrm{O}_{3}$ & 0.0051 & 0.0050 & 0.0051 & 0.0001 & 1.4 & -2.0 \\
\hline $\mathrm{CoO}$ & 0.0001 & 0.0001 & 0.0001 & 0.0000 & 0.0 & 0.0 \\
\hline $\mathrm{CuO}$ & 0.0003 & 0.0003 & 0.0003 & 0.0000 & 0.0 & 0.0 \\
\hline $\mathrm{La}_{2} \mathrm{O}_{3}$ & 0.0027 & 0.0027 & 0.0027 & 0.0000 & 0.0 & 0.0 \\
\hline $\mathrm{Nd}_{2} \mathrm{O}_{3}$ & 0.0020 & 0.0020 & 0.0020 & 0.0000 & 0.0 & 0.0 \\
\hline $\mathrm{SnO}_{2}$ & 0.0019 & 0.0020 & 0.0020 & 0.0001 & 3.6 & 5.3 \\
\hline LOI & 0.0188 & 0.0199 & 0.0194 & 0.0008 & 4.0 & 5.9 \\
\hline Total & 0.9836 & 0.9871 & 0.9854 & 0.0025 & 0.3 & 0.4 \\
\hline
\end{tabular}

SD: Standard deviation; RSD: Relative standard deviation (SD/Average); RPD: Relative percent difference between analyzed compositions; BRL: Below reporting limit; (-) Empty field; LOI: Loss on ignition at $1000^{\circ} \mathrm{C}$. 
Table 4.10. Analyzed Composition of EMSP-Ni1.5 (Chem.)-1M Glass after First Melt Compared with Target Values for Designed Ni1.5 Glass

\begin{tabular}{|c|c|c|c|c|c|c|}
\hline Component & Ni1.5 & Ni1.5-1M & Average & SD & RSD, $\%$ & RPD, \% \\
\hline $\mathrm{Al}_{2} \mathrm{O}_{3}$ & 0.0814 & 0.0771 & 0.0793 & 0.0030 & 3.8 & -5.3 \\
\hline $\mathrm{B}_{2} \mathrm{O}_{3}$ & 0.0792 & 0.0782 & 0.0787 & 0.0007 & 0.9 & -1.3 \\
\hline $\mathrm{BaO}$ & 0.0009 & 0.0009 & 0.0009 & 0.0000 & 0.0 & 0.0 \\
\hline $\mathrm{CaO}$ & 0.0057 & 0.0058 & 0.0058 & 0.0001 & 1.2 & 1.8 \\
\hline $\mathrm{CdO}$ & 0.0064 & 0.0060 & 0.0062 & 0.0003 & 4.6 & -6.3 \\
\hline $\mathrm{Cr}_{2} \mathrm{O}_{3}$ & 0.0017 & 0.0017 & 0.0017 & 0.0000 & 0.0 & 0.0 \\
\hline F & 0.0001 & NM & - & - & - & - \\
\hline $\mathrm{Fe}_{2} \mathrm{O}_{3}$ & 0.1438 & 0.1444 & 0.1441 & 0.0004 & 0.3 & 0.4 \\
\hline $\mathrm{K}_{2} \mathrm{O}$ & 0.0034 & 0.0037 & 0.0036 & 0.0002 & 6.0 & 8.8 \\
\hline $\mathrm{Li}_{2} \mathrm{O}$ & 0.0197 & 0.0184 & 0.0191 & 0.0009 & 4.8 & -6.6 \\
\hline $\mathrm{MgO}$ & 0.0013 & 0.0011 & 0.0012 & 0.0001 & 11.8 & -15.4 \\
\hline $\mathrm{MnO}$ & 0.0035 & 0.0036 & 0.0036 & 0.0001 & 2.0 & 2.9 \\
\hline $\mathrm{Na}_{2} \mathrm{O}$ & 0.1850 & 0.1725 & 0.1788 & 0.0088 & 4.9 & -6.8 \\
\hline $\mathrm{NiO}$ & 0.0150 & 0.0148 & 0.0149 & 0.0001 & 0.9 & -1.3 \\
\hline $\mathrm{P}_{2} \mathrm{O}_{5}$ & 0.0032 & 0.0035 & 0.0034 & 0.0002 & 6.3 & 9.4 \\
\hline $\mathrm{Rh}_{2} \mathrm{O}_{3}$ & 0.0003 & 7.1E-05 & $1.9 \mathrm{E}-04$ & $1.6 \mathrm{E}-04$ & 87.3 & -76.3 \\
\hline $\mathrm{RuO}_{2}$ & $2.9 \mathrm{E}-05$ & $2.0 \mathrm{E}-05$ & $2.5 \mathrm{E}-05$ & $6.4 \mathrm{E}-06$ & 26.0 & -31.0 \\
\hline $\mathrm{SiO}_{2}$ & 0.3996 & 0.4129 & 0.4063 & 0.0094 & 2.3 & 3.3 \\
\hline $\mathrm{SO}_{3}$ & 0.0008 & 0.0008 & 0.0008 & 0.0000 & 0.0 & 0.0 \\
\hline $\mathrm{TiO}_{2}$ & 0.0003 & 0.0004 & 0.0004 & 0.0001 & 20.2 & 33.3 \\
\hline $\mathrm{ZnO}$ & 0.0002 & 0.0002 & 0.0002 & 0.0000 & 0.0 & 0.0 \\
\hline $\mathrm{ZrO}_{2}$ & 0.0412 & 0.0380 & 0.0396 & 0.0023 & 5.7 & -7.8 \\
\hline $\mathrm{Cl}$ & 0.0002 & NM & - & - & - & - \\
\hline $\mathrm{Ce}_{2} \mathrm{O}_{3}$ & 0.0020 & 0.0021 & 0.0021 & 0.0001 & 3.4 & 5.0 \\
\hline $\mathrm{CoO}$ & 0.0001 & 0.0001 & 0.0001 & 0.0000 & 0.0 & 0.0 \\
\hline $\mathrm{CuO}$ & 0.0004 & 0.0004 & 0.0004 & 0.0000 & 0.0 & 0.0 \\
\hline $\mathrm{La}_{2} \mathrm{O}_{3}$ & 0.0022 & 0.0022 & 0.0022 & 0.0000 & 0.0 & 0.0 \\
\hline $\mathrm{Nd}_{2} \mathrm{O}_{3}$ & 0.0018 & 0.0016 & 0.0017 & 0.0001 & 8.3 & -11.1 \\
\hline $\mathrm{SnO}_{2}$ & 0.0010 & 0.0008 & 0.0009 & 0.0001 & 15.7 & -20.0 \\
\hline LOI & - & 0.0168 & - & - & - & - \\
\hline Total & 1.0000 & 1.0081 & - & - & - & - \\
\hline
\end{tabular}

SD: Standard deviation; RSD: Relative standard deviation (SD/Average); RPD: Relative percent difference between analyzed and designed compositions; NM: Not measured; (-) Empty field; LOI: Loss on ignition at $1000^{\circ} \mathrm{C}$. 
Table 4.11. Analyzed Composition of Baseline Glass EMSP-Ni1.5-(Chem.)-1M, After First Melt, Compared with Analyzed Composition of Baseline Glass EMSP-Ni1.5-7D, After 7 Days at $850^{\circ} \mathrm{C}$ (Double Crucible Test)

\begin{tabular}{|c|c|c|c|c|c|c|}
\hline Component & Ni1.5-1M & Ni1.5-7D & Average & SD & RSD, \% & RPD, \% \\
\hline $\mathrm{Al}_{2} \mathrm{O}_{3}$ & 0.0771 & 0.0780 & 0.0776 & 0.0006 & 0.8 & 1.2 \\
\hline $\mathrm{B}_{2} \mathrm{O}_{3}$ & 0.0782 & 0.0757 & 0.0770 & 0.0018 & 2.3 & -3.2 \\
\hline $\mathrm{BaO}$ & 0.0009 & 0.0009 & 0.0009 & 0.0000 & 0.0 & 0.0 \\
\hline $\mathrm{CaO}$ & 0.0058 & 0.0059 & 0.0059 & 0.0001 & 1.2 & 1.7 \\
\hline $\mathrm{CdO}$ & 0.0060 & 0.0059 & 0.0060 & 0.0001 & 1.2 & -1.7 \\
\hline $\mathrm{Cr}_{2} \mathrm{O}_{3}$ & 0.0017 & 0.0017 & 0.0017 & 0.0000 & 0.0 & 0.0 \\
\hline $\mathrm{Fe}_{2} \mathrm{O}_{3}$ & 0.1444 & 0.1473 & 0.1459 & 0.0021 & 1.4 & 2.0 \\
\hline $\mathrm{K}_{2} \mathrm{O}$ & 0.0037 & 0.0037 & 0.0037 & 0.0000 & 0.0 & 0.0 \\
\hline $\mathrm{Li}_{2} \mathrm{O}$ & 0.0184 & 0.0180 & 0.0182 & 0.0003 & 1.6 & -2.2 \\
\hline $\mathrm{MgO}$ & 0.0011 & 0.0010 & 0.0011 & 0.0001 & 6.7 & -9.1 \\
\hline $\mathrm{MnO}$ & 0.0036 & 0.0036 & 0.0036 & 0.0000 & 0.0 & 0.0 \\
\hline $\mathrm{Na}_{2} \mathrm{O}$ & 0.1725 & 0.1698 & 0.1712 & 0.0019 & 1.1 & -1.6 \\
\hline $\mathrm{NiO}$ & 0.0148 & 0.0149 & 0.0149 & 0.0001 & 0.5 & 0.7 \\
\hline $\mathrm{P}_{2} \mathrm{O}_{5}$ & 0.0035 & 0.0034 & 0.0035 & 0.0001 & 2.0 & -2.9 \\
\hline $\mathrm{Rh}_{2} \mathrm{O}_{3}$ & 7.1E-05 & $7.2 \mathrm{E}-05$ & 7.2E-05 & 7.1E-07 & 1.0 & 1.4 \\
\hline $\mathrm{RuO}_{2}$ & $2.0 \mathrm{E}-05$ & $3.5 \mathrm{E}-06$ & $1.2 \mathrm{E}-05$ & $1.2 \mathrm{E}-05$ & 99.3 & -82.5 \\
\hline $\mathrm{SiO}_{2}$ & 0.4129 & 0.4172 & 0.4151 & 0.0030 & 0.7 & 1.0 \\
\hline $\mathrm{SO}_{3}$ & 0.0008 & 0.0009 & 0.0009 & 0.0001 & 8.3 & 12.5 \\
\hline $\mathrm{TiO}_{2}$ & 0.0004 & 0.0004 & 0.0004 & 0.0000 & 0.0 & 0.0 \\
\hline $\mathrm{ZnO}$ & 0.0002 & 0.0002 & 0.0002 & 0.0000 & 0.0 & 0.0 \\
\hline $\mathrm{ZrO}_{2}$ & 0.0380 & 0.0384 & 0.0382 & 0.0003 & 0.7 & 1.1 \\
\hline $\mathrm{Ce}_{2} \mathrm{O}_{3}$ & 0.0021 & 0.0039 & 0.0030 & 0.0013 & 42.4 & 85.7 \\
\hline $\mathrm{CoO}$ & 0.0001 & 0.0001 & 0.0001 & 0.0000 & 0.0 & 0.0 \\
\hline $\mathrm{CuO}$ & 0.0004 & 0.0004 & 0.0004 & 0.0000 & 0.0 & 0.0 \\
\hline $\mathrm{La}_{2} \mathrm{O}_{3}$ & 0.0022 & 0.0019 & 0.0021 & 0.0002 & 10.3 & -13.6 \\
\hline $\mathrm{Nd}_{2} \mathrm{O}_{3}$ & 0.0016 & 0.0017 & 0.0017 & 0.0001 & 4.3 & 6.2 \\
\hline $\mathrm{SnO}_{2}$ & 0.0008 & 0.0008 & 0.0008 & 0.0000 & 0.0 & 0.0 \\
\hline LOI & 0.0168 & 0.0241 & 0.0205 & 0.0052 & 25.2 & 43.5 \\
\hline Total & 1.0081 & 1.0199 & 1.0140 & 0.0083 & 0.8 & 1.2 \\
\hline
\end{tabular}

SD: Standard deviation; RSD: Relative standard deviation (SD/Average); RPD: Relative percent difference between analyzed compositions; BRL: Below reporting limit; LOI: Loss on ignition at $1000^{\circ} \mathrm{C}$. 



\subsection{Experimental Methods}

\subsection{Optical Microscopy}

Partially reacted feed or glass samples were removed from Pt-10\% Rh boxes, cross-sectioned, and polished with a variable-speed polisher by using silicon carbide sandpapers $(240,320,600$, and 1200 grit sizes) and diamond suspensions $(3,1$, and $0.25 \mu \mathrm{m})$. Samples werre analyzed with a stereo microscope (magnification range from $10 \times$ to $70 \times$ ) or high-magnification microscope with up to $1000 \times$ magnification under reflective or transmitted light. The size and surface fraction of spinel crystals in selected samples was determined with an image-analysis program, Clemex $3.0^{\circledR}$.

\subsection{XRD}

Partially reacted feed or glass samples were removed from $\mathrm{Pt}-10 \% \mathrm{Rh}$ boxes and ground for 2 min in a tungsten carbide mill. Approximately $1 \mathrm{~g}$ of sample powder was mixed with 5 mass $\%$ of internal standard $\left(\mathrm{CaF}_{2}\right)$ for $1 \mathrm{~min}$ in a tungsten carbide mill, mounted in an X-ray diffraction (XRD) sample holder, and scanned with a Scintag PADV-V equipped with a Peltier detector and $\mathrm{Cu}$ target (Applied Process Engineering Laboratory [APEL]-PAD-V ${ }^{(a)}$ ). The scan parameters were step size $0.03^{\circ} 2-\theta$, dwell time 4 secons, and scan range from 5 to $70^{\circ} 2-\theta$. Jade and RIQAS 3.1 software wase used to identify crystalline phases and to determine their concentration in analyzed samples following the PNNL procedure Glass Development Laboratory (GDL)-XRD. ${ }^{\text {(b) }}$

\subsection{SEM-EDS}

Thin sections of glass samples were sputter coated with $\mathrm{Au} / \mathrm{Pd}$ and analyzed with a backscattered electron detector. The working distance was $\sim 12 \mathrm{~mm}$, the voltage was $15 \mathrm{kV}$, and the spot size ranged from 42 to 45 .

(a) APEL-PAD-V, Rev. 2. 2002. Operation of Scintag Pad-VX-Ray Diffractometer. Safe Operating Procedure.

(b) GDL-XRD. Rev. 1. 2007. Quantitative and Semi-quantitative analysis using X-Ray Diffraction, Pacific Northwest National Laboratory, Technical Procedure. 



\subsection{Liquidus Temperature}

The liquidus temperature $\left(T_{L}\right)$ of 25 EMSP glasses was obtained through heat-treatments of glasses at selected temperatures for $24 \pm 2$ hours in Pt-alloy boxes with tight fitting lids (to avoid volatility) in accordance with the PNNL procedure GDL-LQT. ${ }^{\text {(a) }}$ Glass samples were quenched, cross-sectioned, polished, and analyzed with a high-magnification optical microscope for the presence of crystals. The $T_{L}$ was determined within $7^{\circ} \mathrm{C}$ of the temperature range between the highest and the lowest temperature at which a glass sample contains or does not contain spinel crystals in the glass, respectively.

Table 6.1 summarizes determined $T_{L}$ 's for all tested glasses. Glasses batched using AZ-101 simulant have significantly lower $T_{L}$ 's than glasses of presumably the same composition that were prepared from chemicals. The standard deviation varied from $\sim 41$ to $\sim 99^{\circ} \mathrm{C}$. This variation can be explained by slightly different glass compositions of glasses prepared from chemicals because they were formulated using the composition of AZ-101 simulant as given by Eibling. Our analysis of this simulant that is shown in Table 4.1 indicated discrepancies between Eibling's composition of AZ-101 and the analytically determined composition. The concentrations for some components were different by more than $20 \%$. Other factors that contributed to considerably higher $T_{L}$ 's of glasses prepared from chemicals are $\mathrm{Rh}_{2} \mathrm{O}_{3}$ and a different chemical make-up. These glasses, except EMSP-BL-w/o nm-(Chem.), contained $\sim 0.03$ wt $\%$ of $\mathrm{Rh}_{2} \mathrm{O}_{3}$ and were batched using different oxides. Additions of $\mathrm{Rh}_{2} \mathrm{O}_{3}$ increased the $T_{L}$ significantly, but no further increase in $T_{L}$ was observed for higher concentrations of $\mathrm{RuO}_{2}(0.029$ and $0.087 \mathrm{wt} \%)$ in EMSP-Ni1.5-0.029 and 0.087 wt\%-(Chem.) glasses. In fact, the $T_{L}$ of these glasses was $38^{\circ} \mathrm{C}$ lower than that of EMSP-Ni1.5-(Chem.). All the components that were added to the baseline glass [EMSP-BL-(AZ101 or Chem.)] increased the $T_{L}$ 's. For AZ-101 glasses, the $T_{L}$ 's increased as follows: $\mathrm{Zn} 0.6<\mathrm{Mn} 1<$ Ni1.07 $<$ Cr0.6 < Mn2.5 < Ni1.5 < Cr1.2 < Fe20 < Ni1.5/nm < Ni1.5/A112 < Fe20/Ni1.5. For glasses prepared from chemicals, the $T_{L}$ 's increased as follows: Ni1.07 $<\mathrm{Mn} 1<\mathrm{Cr} 0.6<\mathrm{Mn} 2.5<\mathrm{Ni1} .5 / 0.029$ and $0.087 \mathrm{wt} \% \mathrm{~nm}<\mathrm{Fe} 20<\mathrm{Cr} 1.2<\mathrm{Ni} 1.5<\mathrm{Ni1} .5 / \mathrm{A} 112<\mathrm{Fe} 20 / \mathrm{Ni} 1.5$. Adding Ni, Fe, and Al to baseline glasses [EMSP-BL-(AZ-101) and (Chem.)] increased the $T_{L}$ 's the most. Obtained $T_{L}$ 's were compared to the $T_{L}$ 's summarized in Table 2.5 and Table 2.6 that were calculated with a new spinel $T_{L}$ model (2003). ${ }^{(\text {b) }}$ For some glasses, mostly from chemicals, the predicted $T_{L}$ 's were off from 4 to $17^{\circ} \mathrm{C}$, but the majority of them exhibited significantly higher differences. The results suggest that this model does not seem to account for the effect of $\mathrm{Rh}_{2} \mathrm{O}_{3}$ on $T_{L}$.

(a) GDL-LQT, Rev. 4. 2007. Standard Test Methods for Determining the Liquidus Temperature (TL) of Waste Glasses and Simulated Waste Glasses, Pacific Northwest National Laboratory, Technical Procedure.

(b) JD Vienna. 2003. New spinel TL, $2003^{\circ} \mathrm{C}$. Not published. 
Table 6.1. Determined $T_{L}$ 's

\begin{tabular}{cccc}
\hline Glass (AZ-101) & $T_{L},{ }^{\circ} \mathrm{C}$ & Glass (Chem.) & $T_{L},{ }^{\circ} \mathrm{C}$ \\
\hline EMSP-BL-(AZ-101) & 967 & EMSP-BL-(Chem.) & 1114 \\
EMSP-Cr0.6-(AZ-101) & 1020 & EMSP-BL-w/o nm-(Chem.) & 1025 \\
EMSP-Cr1.2-(AZ-101) & 1093 & EMSP-Cr0.6-(Chem.) & 1122 \\
EMSP-Ni1.07-(AZ-101) & 1017 & EMSP-Cr1.2-(Chem.) & 1178 \\
EMSP-Ni1.5-(AZ-101) & 1080 & EMSP-Ni1.07-(Chem.) & 1118 \\
EMSP-Fe20-(AZ-101) & 1107 & EMSP-Ni1.5-(Chem.) & 1193 \\
EMSP-Fe20/Ni1.5-(AZ-101) & 1195 & EMSP-Fe20-(Chem.) & 1168 \\
EMSP-Zn0.6-(AZ-101) & 979 & EMSP-Fe20/Ni1.5-(Chem.) & 1241 \\
EMSP-Mn1-(AZ-101) & 996 & EMSP-Mn1-(Chem.) & 1122 \\
EMSP-Mn2.5-(AZ-101) & 1037 & EMSP-Mn2.5-(Chem.) & 1136 \\
EMSP-Ni1.5/Al12-(AZ-101) & 1165 & EMSP-Ni1.5/A112-(Chem.) & 1224 \\
EMSP-Ni1.5/nm-(AZ-101) & 1124 & EMSP-Ni1.5/0.029 RuO 2 -(Chem.) & 1155 \\
& & EMSP-Ni1.5/0.087 RuO-(Chem.) & 1155 \\
\hline
\end{tabular}




\subsection{Crystal Growth Rate Test}

Glass chips that were prepared according to liquidus temperature procedure GDL-LQT have been used to determine the average crystal size and concentration of spinel crystals as a function of time and glass composition. To avoid reboil and to eliminate the effects of temperature history and surface nucleation, the selected glasses were heated up in Pt-alloy boxes at $5^{\circ} \mathrm{C} / \mathrm{min}$ from $900^{\circ} \mathrm{C}$ to $1200^{\circ} \mathrm{C}$ with a 30-min dwell time. Then, the boxes were transferred to another furnace for isothermal heat-treatment at 850,900 , and $950^{\circ} \mathrm{C}$ for varying amounts of time, from 1 to 48 hours. Glass samples were analyzed with optical microscopy, XRD, and scanning electron microscopy-energy dispersive spectroscopy (SEMEDS).

\subsection{X-ray Diffraction Analysis}

Table 7.1 through Table 7.7 summarize the changes in concentration of spinel crystals with time and temperature for selected AZ-101 glasses. Not surprisingly, adding spinel-forming components into baseline glass increased the amount of precipitated spinel. The highest concentration of more than $4 \mathrm{wt} \%$ of spinel was observed for Fe/Ni-rich and Fe-rich glasses. In the beginning, the concentration of spinel in $\mathrm{Fe} / \mathrm{Ni}$-rich glass gradually increased from $1.4 \mathrm{wt} \%$ after $1 \mathrm{~h}$ to $4.1 \mathrm{wt} \%$ after $24 \mathrm{~h}$ at $850^{\circ} \mathrm{C}$ and then remained approximately constant for the next 2 days. The same trend was observed for Fe-rich glass, but the constant maximum concentration of spinel decreased to $\sim 2.7 \mathrm{wt} \%$. Figure 7.1 shows the change of spinel and hematite concentration over time for EMSP-Fe20 (AZ-101) glass heat-treated at $850^{\circ} \mathrm{C}$. Figure 7.2 visualizes the formation of less than $10-\mu \mathrm{m}$ spinel crystals from the fast-growing thin platelets of hematite. Doubling the concentration of chromium resulted in more than 5 times higher concentration of spinel in the glass samples reaching the maximum of about 1.1 to $1.2 \mathrm{wt} \%$. Compared to baseline glass, only a small increase, $\sim 0.2 \mathrm{wt} \%$, in spinel concentration was observed for Ni- rich glasses.

Table 7.1. Concentration of Spinel in wt $\%$ at Different Temperatures and Times for EMSP-BL-(AZ-101)

\begin{tabular}{ccccccc}
\hline \multicolumn{7}{c}{ EMSP-BL-(AZ-101) } \\
\hline Temperature, ${ }^{\circ} \mathrm{C}$ & 1 & 3 & 6 & 12 & 24 & 48 \\
\hline 850 & 0 & 0 & - & 0 & 0.1 & 0.2 \\
900 & 0 & 0 & trace & 0.2 & 0 & trace \\
950 & - & - & - & - & - & 0 \\
\hline
\end{tabular}

Table 7.2. Concentration of Spinel in wt\% at Different Temperatures and Times for EMSP-Cr0.6(AZ-101)

\begin{tabular}{ccccccc}
\hline \multicolumn{7}{c}{ EMSP-Cr0.6-(AZ-101) } \\
\cline { 2 - 7 } Temperature, ${ }^{\circ} \mathrm{C}$ & 1 & 3 & 6 & 12 & 24 & 48 \\
\hline 850 & 0.2 & 0.2 & 0.2 & 0.2 & 0.2 & 0.2 \\
900 & 0.1 & 0.1 & 0.1 & 0.1 & 0.2 & 0.2 \\
950 & 0.1 & 0.1 & 0.1 & 0.1 & 0.1 & 0.2 \\
\hline
\end{tabular}


Table 7.3. Concentration of Spinel in wt $\%$ at Different Temperatures and Times for EMSP-Cr1.2(AZ-101)

\begin{tabular}{|c|c|c|c|c|c|c|c|c|c|}
\hline \multicolumn{10}{|c|}{ EMSP-Cr1.2-(AZ-101) } \\
\hline \multirow[b]{2}{*}{ Temperature, ${ }^{\circ} \mathrm{C}$} & \multicolumn{9}{|c|}{ Time, $\mathrm{h}$} \\
\hline & 1 & 3 & 6 & 12 & 24 & 24 & 48 & 48 & 72 \\
\hline 850 & trace & 0.2 & 0.4 & 1 & 1.1 & 1.2 & 1.2 & & 1.1 \\
\hline 900 & trace & 0.3 & 0.9 & 1.2 & 1 & 1 & 1 & 1 & \\
\hline 950 & 0.1 & 0.2 & 0.5 & 0.5 & 0.5 & & 0.5 & & \\
\hline
\end{tabular}

Table 7.4. Concentration of Spinel in wt $\%$ at Different Temperatures and Times for EMSP-Fe20(AZ-101)

\begin{tabular}{ccccccccc}
\hline \multicolumn{10}{c}{ EMSP-Fe20-(AZ-101) } \\
\hline & \multicolumn{1}{c}{ Time, $\mathrm{h}$} \\
\cline { 2 - 10 } Temperature, ${ }^{\circ} \mathrm{C}$ & 1 & 3 & 6 & 12 & 24 & 24 & 48 & 72 \\
\hline 850 & 0.1 & 0.4 & & 1.9 & 2.7 & & 2.9 & \\
900 & 0 & 0.1 & 0.3 & 0.6 & 0.8 & & 0.9 & 1 \\
950 & 0.2 & 0.3 & 0.3 & 0.5 & 0.8 & 0.7 & 1.1 & \\
\hline
\end{tabular}

Table 7.5. Concentration of Spinel in wt $\%$ at Different Temperatures and Times for EMSP-Fe20/Ni1.5(AZ-101)

\begin{tabular}{|c|c|c|c|c|c|c|c|c|}
\hline \multicolumn{9}{|c|}{ EMSP-Fe20/Ni1.5-(AZ-101) } \\
\hline \multirow[b]{2}{*}{ Temperature, ${ }^{\circ} \mathrm{C}$} & \multicolumn{8}{|c|}{ Time, $\mathrm{h}$} \\
\hline & 1 & 3 & 6 & 12 & 24 & 48 & 48 & 72 \\
\hline 850 & 1.4 & 1.6 & 2.7 & & 4.1 & 4.3 & & 4.3 \\
\hline 900 & 0.5 & 1.3 & 1.6 & & 2.1 & 2.8 & 2.7 & \\
\hline 950 & 0.6 & 1.4 & & 1.9 & 2 & 2.3 & & \\
\hline
\end{tabular}

Table 7.6. Concentration of Spinel in wt $\%$ at Different Temperatures and Times for EMSP-Ni1.07(AZ-101)

\begin{tabular}{cccccccc}
\hline \multicolumn{7}{c}{ EMSP-Ni1.07-(AZ-101) } \\
\hline & \multicolumn{7}{c}{ Time, $\mathrm{h}$} \\
Temperature, ${ }^{\circ} \mathrm{C}$ & 1 & 3 & 6 & 12 & 24 & 24 & 48 \\
\hline 850 & 0 & 0 & 0.1 & 0.2 & 0.3 & 0.2 & 0.5 \\
900 & 0 & 0.1 & 0 & 0.2 & 0.3 & & 0.3 \\
950 & 0 & 0 & 0 & 0.2 & 0.3 & & 0.1 \\
\hline
\end{tabular}

Table 7.7. Concentration of Spinel in wt $\%$ at Different Temperatures and Times for EMSP-Ni1.5(AZ-101)

\begin{tabular}{cccccccc}
\hline \multicolumn{7}{c}{ EMSP-Ni1.5-(AZ-101) } \\
\hline & 1 & 3 & 6 & 12 & 24 & 48 & 72 \\
\hline & 0.1 & 0.1 & & 0.2 & 0.3 & 0.4 & \\
Temperature, ${ }^{\circ} \mathrm{C}$ & 0 & 0.1 & 0.2 & 0.3 & 0.4 & 0.3 & 0.4 \\
900 & 0 & 0 & 0.1 & 0.3 & 0.3 & 0.4 & \\
\hline 950 & 850 & 7
\end{tabular}




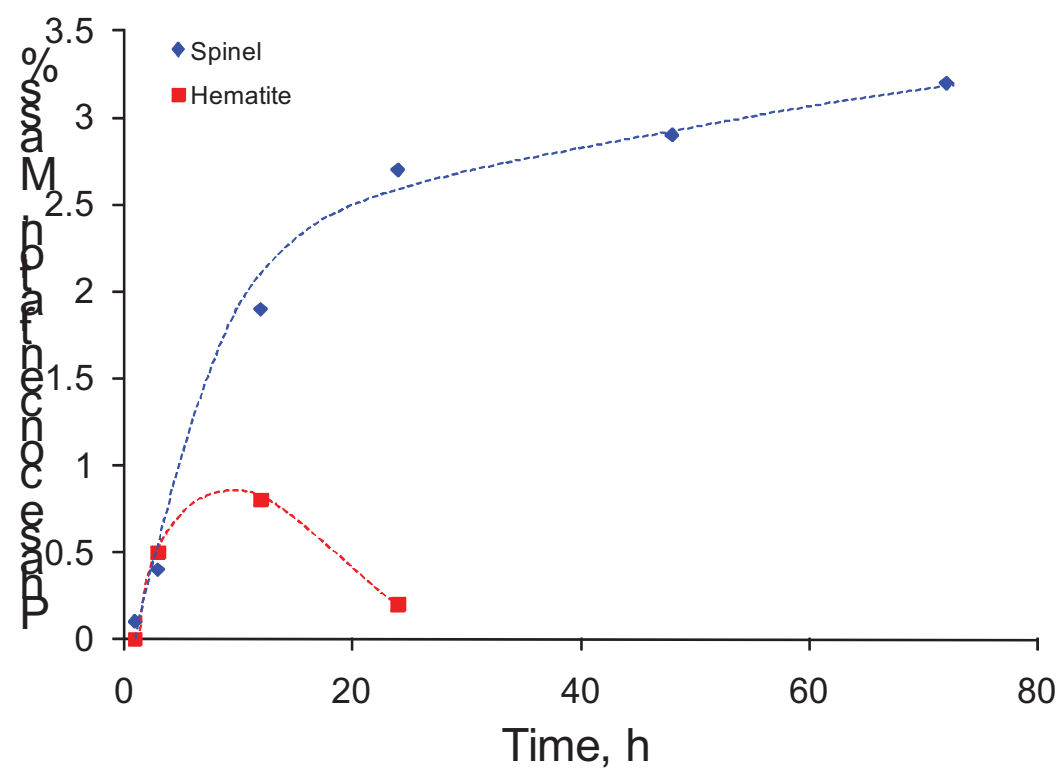

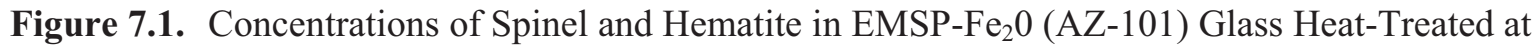
$850^{\circ} \mathrm{C}$ for Different Times

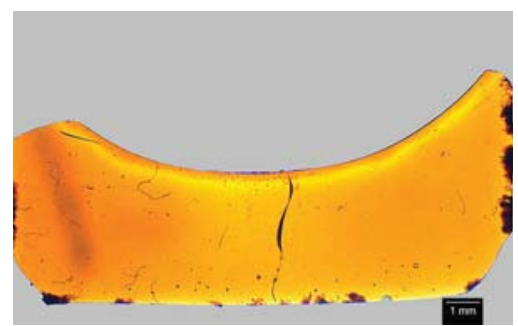

$1 \mathrm{~h}$

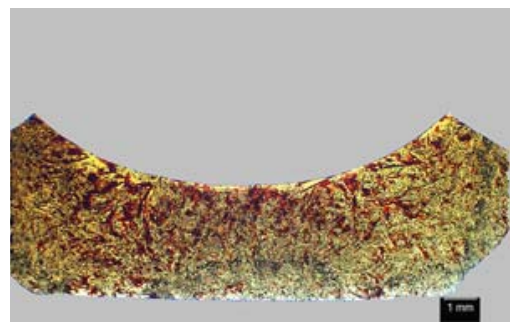

$24 \mathrm{~h}$

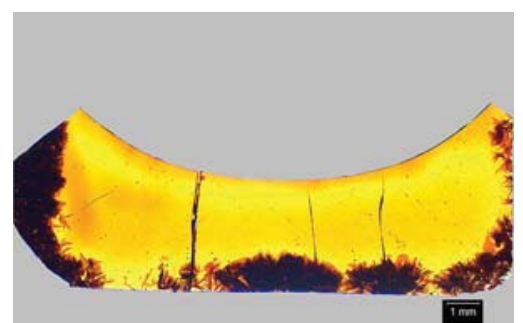

$3 \mathrm{~h}$

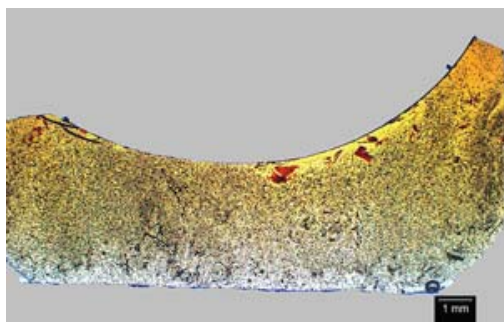

$48 \mathrm{~h}$

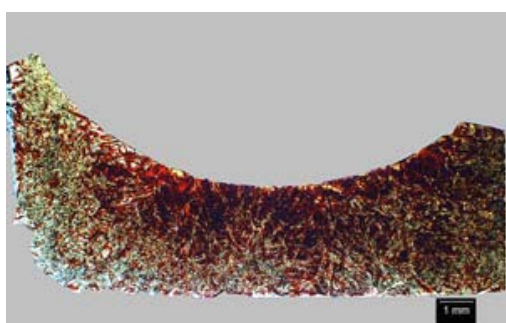

$12 \mathrm{~h}$

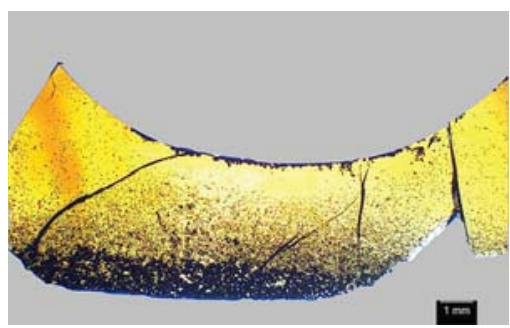

$72 \mathrm{~h}$

Figure 7.2. Optical Images of Hematite (red platelets) and Spinel (black crystals) that Formed in EMSP-Fe 20 (AZ-101) Glass After Heat-Treatments at $850^{\circ} \mathrm{C}$ for Various Times

\subsection{SEM-EDS}

Figure 7.3 through Figure 7.5 show SEM images of spinel crystals and hematite for Fe, Ni-rich Ferich, and Cr-rich glass, respectively. They provide evidence that spinel crystals nucleate on crystals of hematite and then slowly grow from it until all the hematite is consumed. Figure 7.6 shows a SEM image of more than $40-\mu \mathrm{m}$ crystal that formed in Ni-rich glass after $12 \mathrm{~h}$ at $850^{\circ} \mathrm{C}$. The crystals of this size are quite common for this glass. 


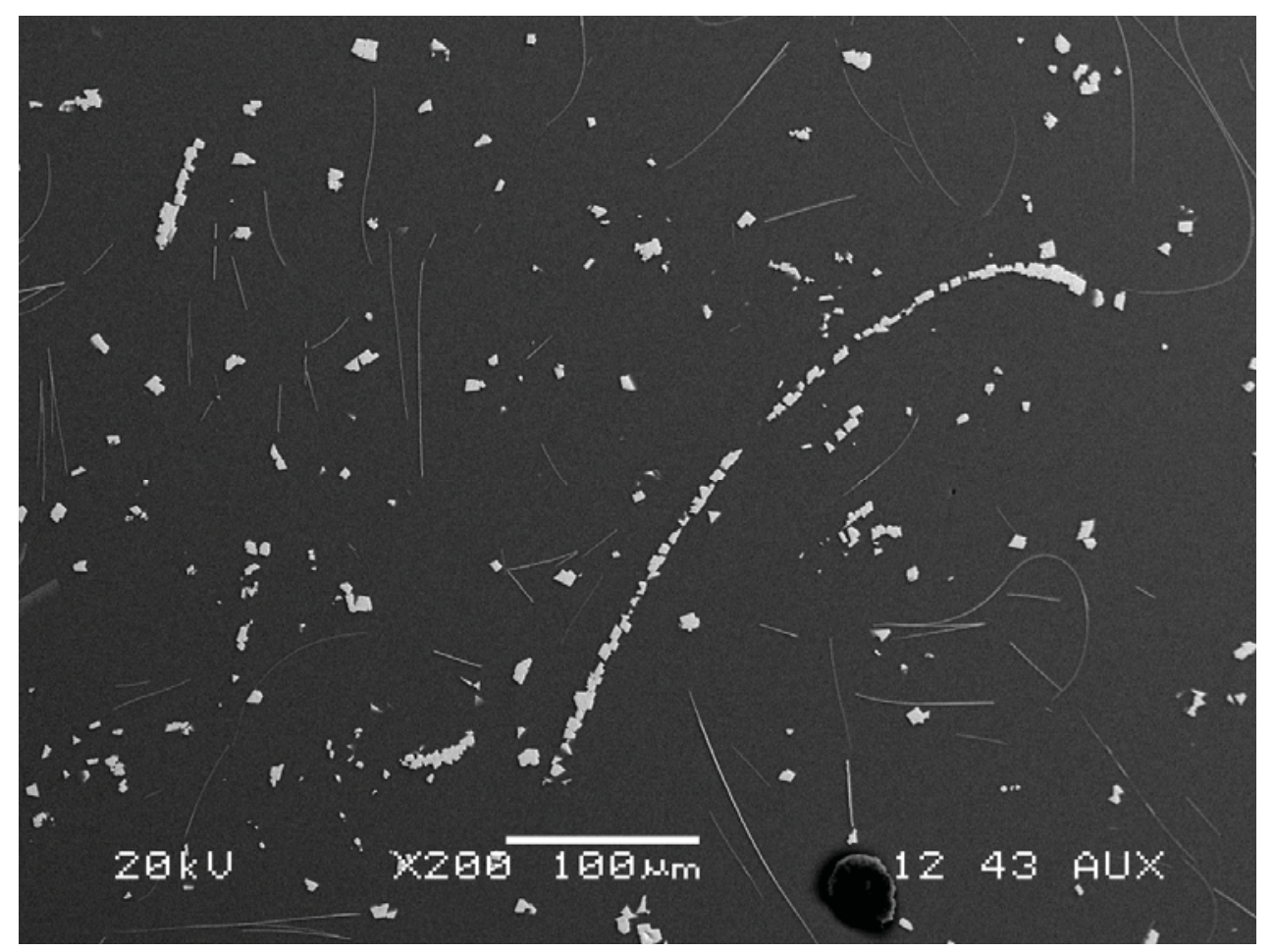

Figure 7.3. SEM Image of Spinel Crystals (White Particles) and Hematite (Hair-Like Crystals) in EMSP-Fe $20-(\mathrm{AZ}-101)$ Glass Heat-Treated at $850^{\circ} \mathrm{C}$ for $12 \mathrm{~h}$

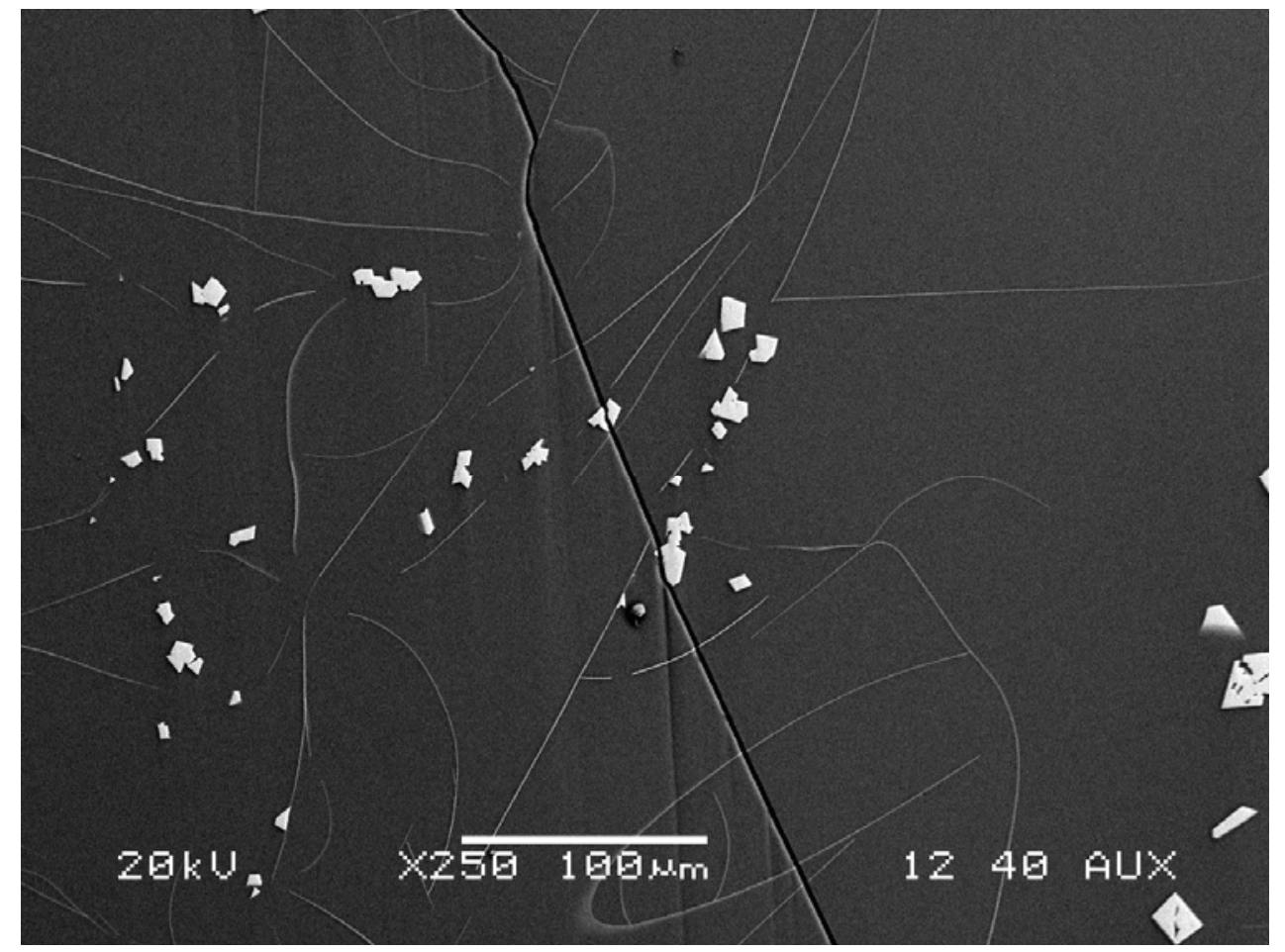

Figure 7.4. SEM Image of Spinel Crystals (White Particles) and Hematite (Hair-Like Crystals) in EMSP-Fe $20 / \mathrm{Ni1.5-(AZ-101)} \mathrm{Glass} \mathrm{Heat-Treated} \mathrm{at} 850^{\circ} \mathrm{C}$ for $6 \mathrm{~h}$ 


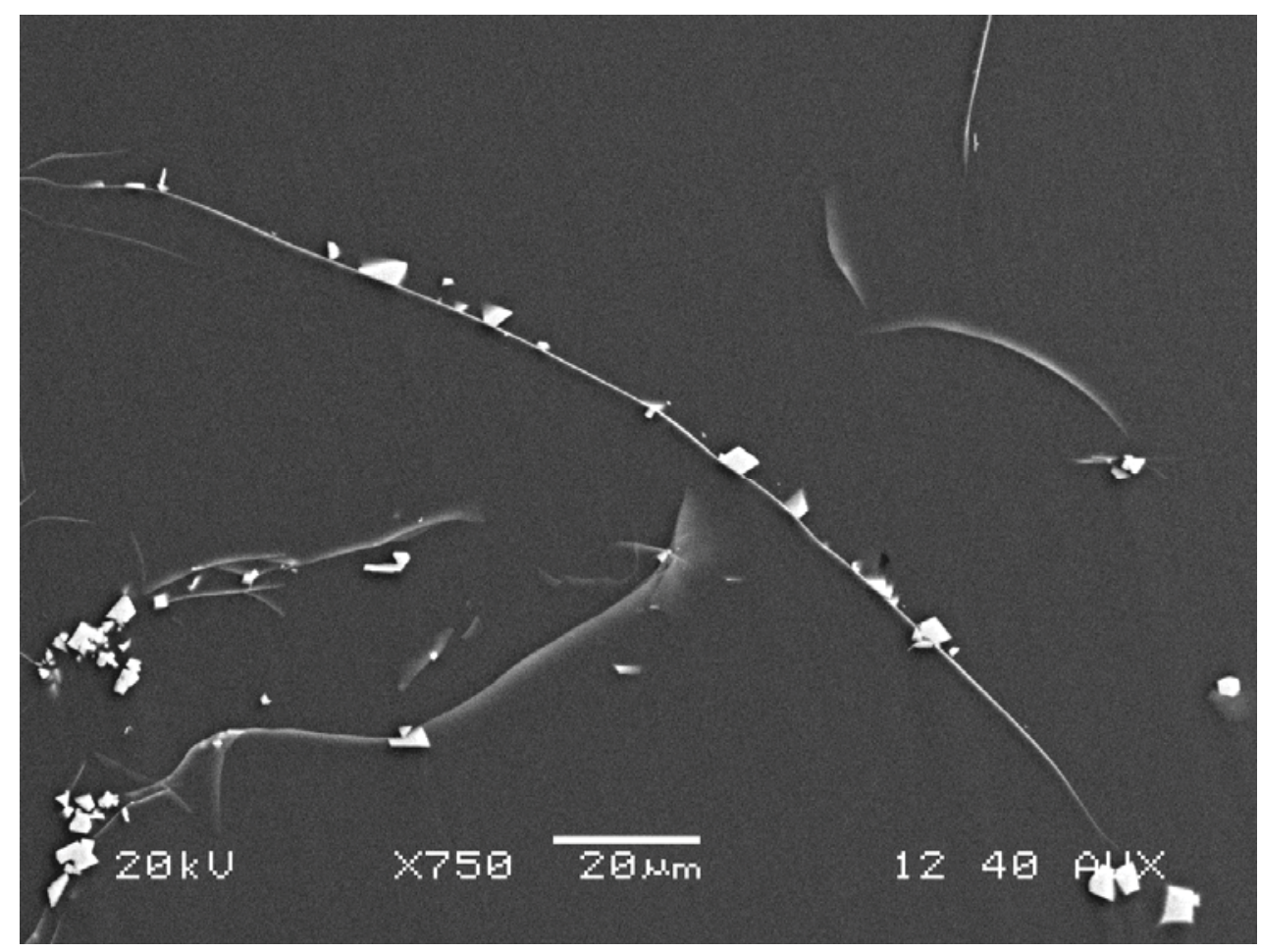

Figure 7.5. SEM Image of Spinel Crystal in EMSP-Cr0.6-(AZ-101) Glass Heat-Treated at $850^{\circ} \mathrm{C}$ for $3 \mathrm{~h}$

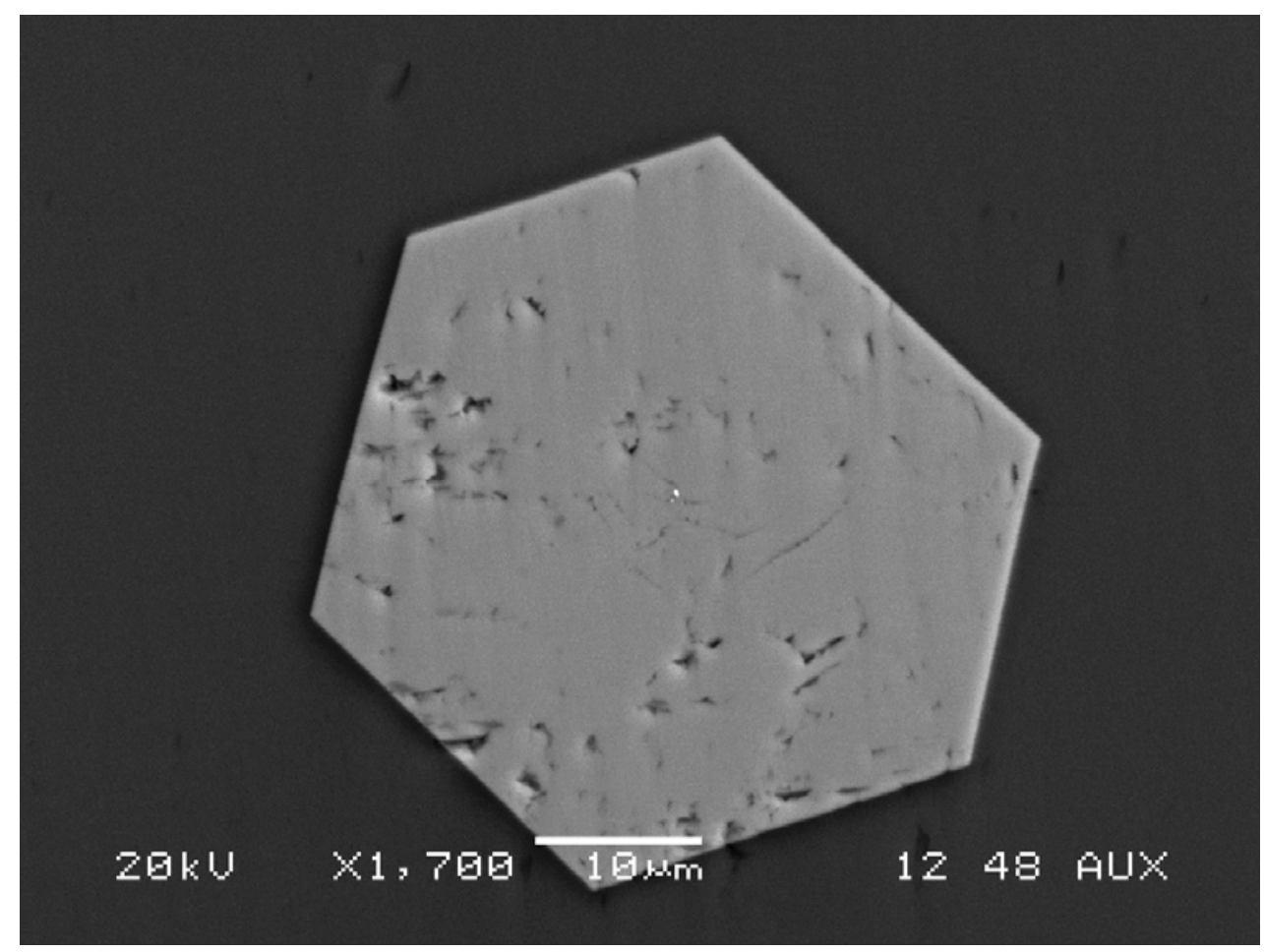

Figure 7.6. SEM Image of Spinel Crystal in EMSP-Ni1.5-(AZ-101) Glass Heat-Treated at $850^{\circ} \mathrm{C}$ for $12 \mathrm{~h}$ 


\subsection{Optical Image Analysis}

Table 7.8 shows the changes in crystal sizes over time for different AZ-101 glasses that were heattreated at $850^{\circ} \mathrm{C}$. The crystals in all glasses continued to grow even after 24 hours. The biggest crystals were observed in Ni-rich glasses. They were bigger than $60 \mu \mathrm{m}$ after $24 \mathrm{~h}$, indicating a fast growing rate of spinel in this glass. The average size of crystals in Cr-rich glass was almost identical for the first $24 \mathrm{~h}$ with crystals that precipitated from EMSP-Ni1.07-(AZ-101) glass but then fell behind by more than $15 \mu \mathrm{m}$ after 48 hours. It was confirmed that additions of iron to Ni-rich glass will decrease the size of precipitated spinel crystals.

Table 7.8. Crystal Size of Spinel Crystals in $\mu \mathrm{m}$ for Various AZ-101 Glasses that Were Heat-Treated at $850^{\circ} \mathrm{C}$ for Different Times

\begin{tabular}{rccccc}
\hline Time, $\mathrm{h}$ & $\begin{array}{c}\text { EMSP-BL- } \\
(\mathrm{AZ}-101)\end{array}$ & $\begin{array}{c}\text { EMSP-Cr0.6- } \\
(\mathrm{AZ}-101)\end{array}$ & $\begin{array}{c}\text { EMSP-Fe20/Ni1.5- } \\
(\mathrm{AZ}-101)\end{array}$ & $\begin{array}{c}\text { EMSP-Ni1.07- } \\
(\mathrm{AZ}-101)\end{array}$ & $\begin{array}{c}\text { EMSP-Ni1.5- } \\
(\mathrm{AZ}-101)\end{array}$ \\
\hline 1 & 9.6 & 8.9 & 8.4 & & \\
3 & & & 20 & & \\
6 & & 14.5 & & 13.5 & \\
12 & & & & & \\
24 & 28 & 44.4 & & 46.5 & 62.4 \\
48 & 40.6 & 48.6 & 29.9 & 63.9 & 72.4 \\
\hline
\end{tabular}




\subsection{Viscosity of EMSP Glasses}

The viscosity of selected EMSP glasses was measured as a function of temperature with a fully automated rotating-spindle Brookfield Digital Viscometer (DV-III) in accordance with the PNNL procedure GDL-VIS. ${ }^{\text {(a) }}$ Approximately $50 \mathrm{~mL}$ of glass, as-measured by EtOH displacement in volumetric cylinder, was transferred into a $\mathrm{Pt} / 10 \% \mathrm{Rh}$ crucible and put through a ramp/soak temperature schedule in a high-temperature Deltech ${ }^{\circledR}$ furnace. The viscosity measurement started at $\sim 1150^{\circ} \mathrm{C}$ and continued at $50^{\circ} \mathrm{C}$ intervals during stepwise cooling until the temperature reached $950^{\circ} \mathrm{C}$; the viscosity measurement was completed by stepwise heating at $50^{\circ} \mathrm{C}$ intervals from $950^{\circ} \mathrm{C}$ to $1150^{\circ} \mathrm{C}$. About a 30 -min soak time was allowed at each temperature. This hysteresis approach allowed the potential impacts of crystallization at lower temperatures and volatization at higher temperatures via duplicate measurements to be assessed. The viscometer was calibrated with a Defense Waste Processing Facility (DWPF) startup frit following the PNNL procedure GDL-VSC. ${ }^{(b)}$

Figure 8.1 and Figure 8.2 show the measured viscosities for selected EMSP glasses that were prepared from AZ-101 simulant or raw chemicals. Table 8.1 shows the viscosities of glasses at 850 , 1050 , and $1200^{\circ} \mathrm{C}$ that were obtained from the linear fits of collected data. Viscosities ranged from $\sim 143$ to $\sim 193 \mathrm{~Pa}$.s at $850^{\circ} \mathrm{C}$, from 11.5 to $\sim 14.5 \mathrm{~Pa}$.s at $1050^{\circ} \mathrm{C}$, and from 2.3 to $3.4 \mathrm{~Pa}$.s at $1200^{\circ} \mathrm{C}$. Small additions of spinel-forming components to baseline glasses [BL- (AZ-101) and (Chem.)] resulted in decreased viscosities at 1050 and $1200^{\circ} \mathrm{C}$ for all tested glasses. Interestingly, the additions of Ni to AZ101 baseline glass decreased the glass viscosity at $850^{\circ} \mathrm{C}$ while additions of $\mathrm{Fe}$ and $\mathrm{Cr}$ increased. For glasses prepared from chemicals, only noble metals decreased the viscosity of baseline glass at $850^{\circ} \mathrm{C}$. Additions of $\mathrm{Cr}$ increased the viscosity only by $\sim 1 \%$ compared to an $\sim 10$ and $18 \%$ increase in viscosity for $\mathrm{Ni}$ and Fe-rich glasses, respectively.

The small additions of spinel-forming components to baseline glasses resulted only in relatively small changes in glass viscosities at $850^{\circ} \mathrm{C}$, suggesting that an average viscosity value can be sufficient for the physical modeling of the settling behavior of spinel crystals in the riser. Different viscosities for different glasses did not play as much of a major role as crystals size did in determining the effects of spinelforming components on the settling rate.

(a) GDL-VIS, Rev. 1. 2003. Standard Viscosity Measurement Procedure for Vitrified Nuclear Waste, Pacific Northwest National Laboratory, Technical Procedure.

(b) GDL-VSC, Rev. 1. 1998. Standard Viscosity Calibration Procedure, Pacific Northwest National Laboratory, Technical Procedure. 


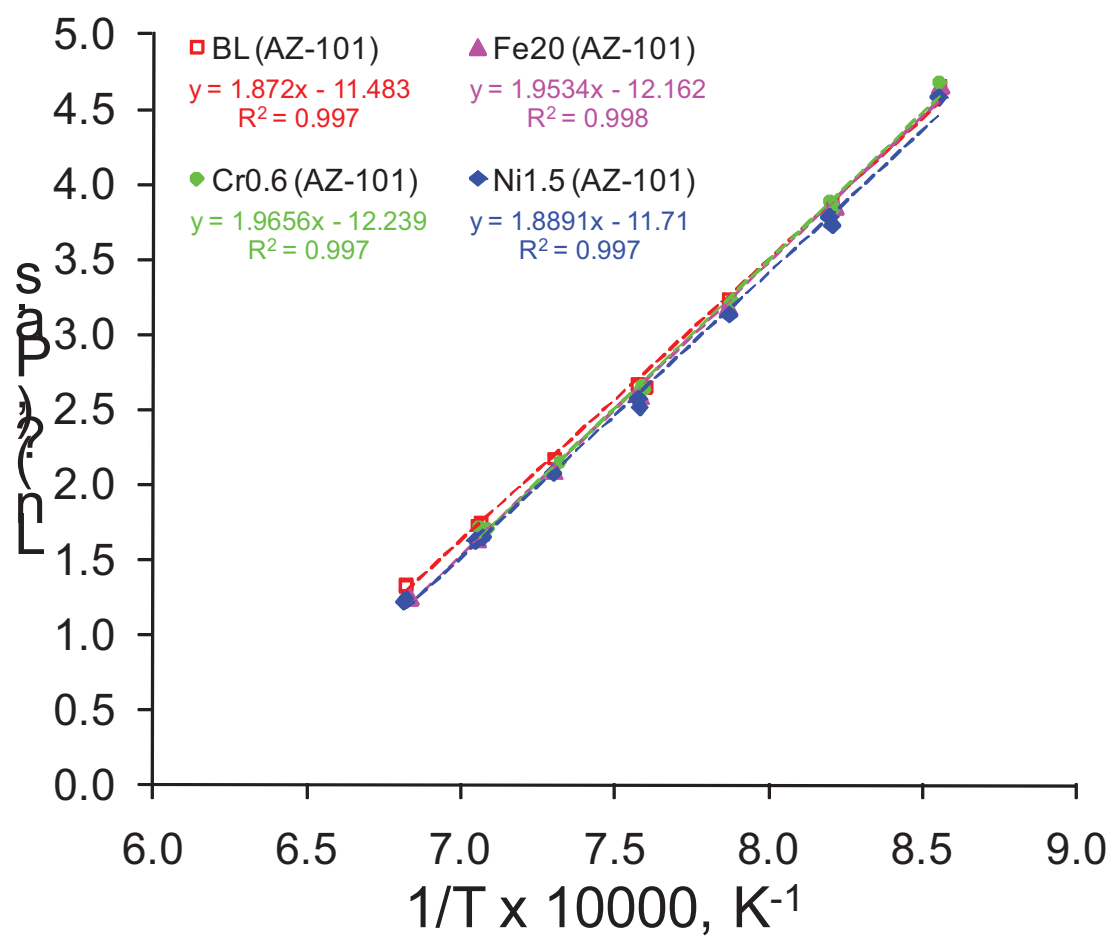

Figure 8.1. Viscosity Curves of EMSP Glasses Prepared from HLW AZ-101 Simulant

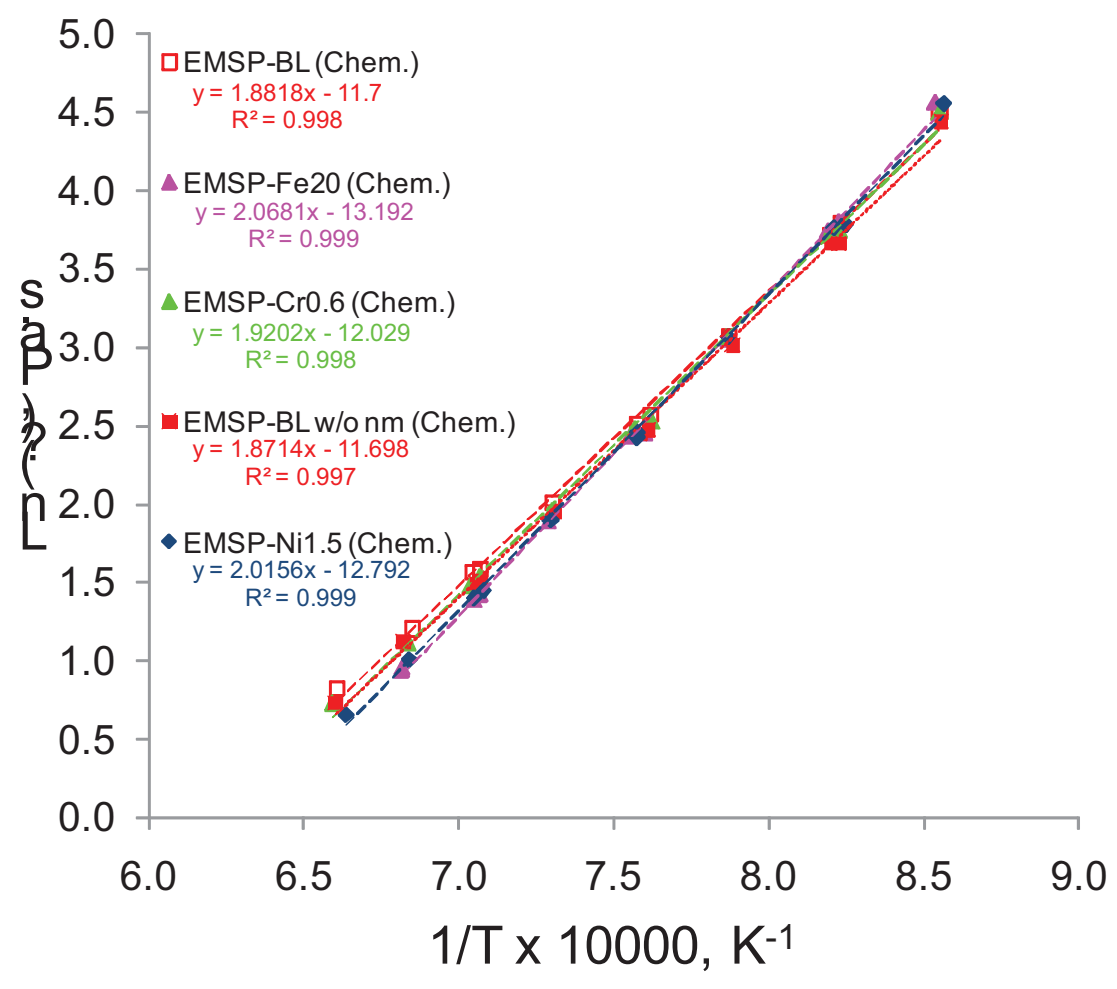

Figure 8.2. Viscosity Curves of EMSP Glasses Prepared from Raw Chemicals 
Table 8.1. Calculated Glass Viscosities at 850,1050 , and $1200^{\circ} \mathrm{C}$

\begin{tabular}{lccc}
\hline Glass & $\eta_{850^{\circ} \mathrm{C}}$, Pa.s & $\eta_{1050^{\circ} \mathrm{C}}$, Pa.s & $\eta_{1200^{\circ} \mathrm{C}}$, Pa.s \\
\hline EMSP-BL-(AZ-101) & 178.5 & 14.4 & 3.4 \\
EMSP-Fe20-(AZ-101) & 186.8 & 13.5 & 3.0 \\
EMSP-Cr0.6-(AZ-101.) & 192.8 & 13.7 & 3.0 \\
EMSP-Ni1.5-(AZ-101) & 165.6 & 13.0 & 3.0 \\
EMSP-BL-(Chem.) & 156.8 & 12.5 & 2.9 \\
EMSP-BL w/o nm-(Chem.) & 143.2 & 11.5 & 2.7 \\
EMSP-Fe20-(Chem.) & 185.2 & 11.5 & 2.3 \\
EMSP-Cr0.6-(Chem.) & 158.8 & 12.0 & 2.7 \\
EMSP-Ni1.5-(Chem.) & 173.1 & 11.5 & 2.4 \\
\hline
\end{tabular}





\subsection{Double Crucible and High-Chromia Crucible Tests}

The settling of spinel crystals in designed EMSP glasses was studied with double-crucible and high-chromia crucible tests with a goal to evaluate the effect of spinel-forming components, noble metals, and alumina on the size and concentration of crystals as well as on the accumulation rate. Figure 9.1 shows the cross-section of the double-crucible assembly and a high-chromia crucible. In the doublecrucible test, the alumina crucible was nested in a larger silica crucible and covered with molten glass to eliminate Marangoni convection in the meniscus and bubble generation at the bottom of silica crucibles (undisturbed settling). The core-drilled second silica crucible held the alumina crucible in place and filled the space, saving more than $0.5 \mathrm{~kg}$ of glass per test. To minimize the effect of the surface crystallization and to eliminate the impact of temperature history on crystal nucleation and growth, powdered glass was melted in a Pt-crucible at $1200^{\circ} \mathrm{C}$ for 1 hour. Then the crucible was removed from the melting furnace, and molten glass was poured into three double crucibles or six high-chromia crucibles that were rested inside the furnace at $850^{\circ} \mathrm{C}$, mimicking the temperature in the glass discharge riser. Double- and highchromia crucibles were removed at various times and cross-sectioned. The marked rectangular pieces shown in Figure 9.1 were cut out from the crucibles, thin-sectioned, and analyzed with SEM-EDS and Clemex image analysis to determine the thickness of the spinel sludge layer and the size and surface fraction of spinel crystals in the accumulated layer.

A

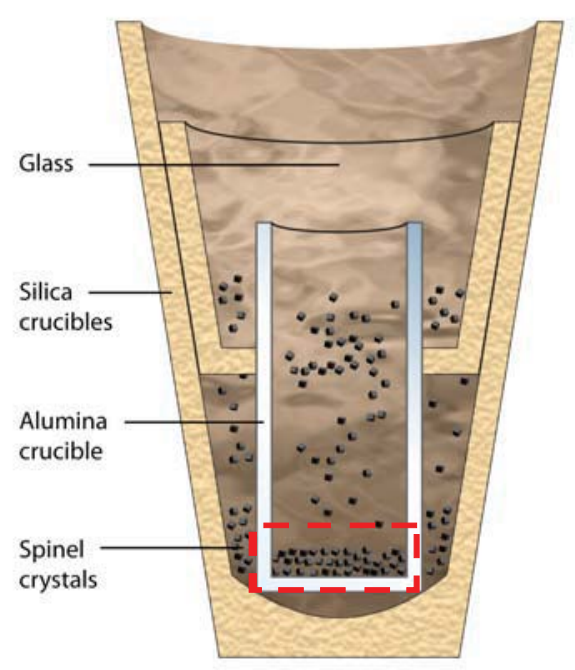

$\mathrm{B}$

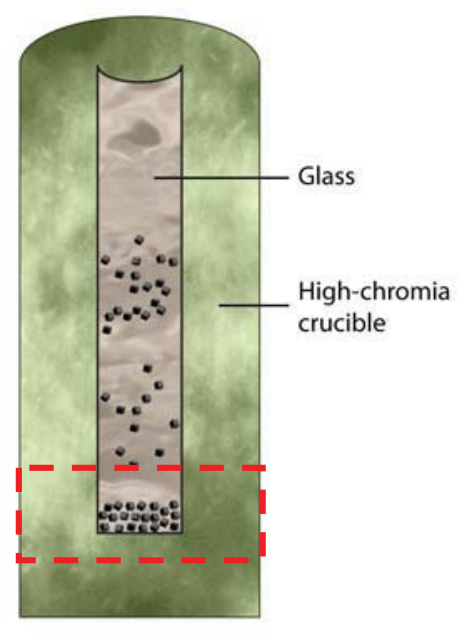

Figure 9.1. Cross-Section of Double Crucible Assembly (A) and High-Chromia Crucible (B) (redmarked area shows the location of thin-sectioned samples)

Figure 9.2 shows the growth of a spinel layer on the bottom of the crucible for four glasses that were heat-treated at $850^{\circ} \mathrm{C}$ for various times. Adding $\sim 1 \mathrm{wt} \%$ of $\mathrm{NiO}$ or $\sim 0.4 \mathrm{wt} \%$ of $\mathrm{Cr}_{2} \mathrm{O}_{3}$ to the baseline glass increased the accumulation rate of spinel crystals by a factor of $\sim 9$ and $\sim 2$, respectively. The high accumulation rate $(\sim 227 \mathrm{~mm} /$ year $)$ of spinel crystals in the glass containing $1.5 \mathrm{wt} \%$ of $\mathrm{NiO}$ is not surprising if we consider the settling of up to $210-\mu \mathrm{m}$-sized crystals. Figure 9.3 shows the time-sequence SEM images of high-Ni glass after heat-treatments at $850^{\circ} \mathrm{C}$, visualizing the growth of the spinel layer as well as the change in size and concentration of crystals in this layer over time. Figure 9.4 details the average crystal size distribution in the top $\sim 2.6$ - $\mathrm{mm}$ spinel layer (Figure 9.3[D, A]) and in the bottom 
$\sim 0.36$-mm spinel layer (Figure 9.3[D, B]) for the same glass after heat-treatment at $850^{\circ} \mathrm{C}$ for 194 hours. The average size of crystals in the bottom layer is about $30 \mu \mathrm{m}$ smaller than the average size of crystals in the top part of the settling layer. Figure 9.5 shows the growth of spinel crystals as the change of average crystal size and the layer compaction over the time in high-Ni glass heat-treated at $850^{\circ} \mathrm{C}$. The nucleated spinel crystals grew fast, reaching $\sim 41 \mu \mathrm{m}$ in just about 67 hours. Adding noble metals to high-Ni glass [EMSP-Ni1.5/nm-(AZ-101)] decreased the spinel layer thickness from $\sim 2.70 \mathrm{~mm}$ to $\sim 0.32 \mathrm{~mm}$ after 7day heat-treatment at $850^{\circ} \mathrm{C}$. The nucleation agents (noble metals) increased the number density of crystals, generating small crystals that settle slowly.

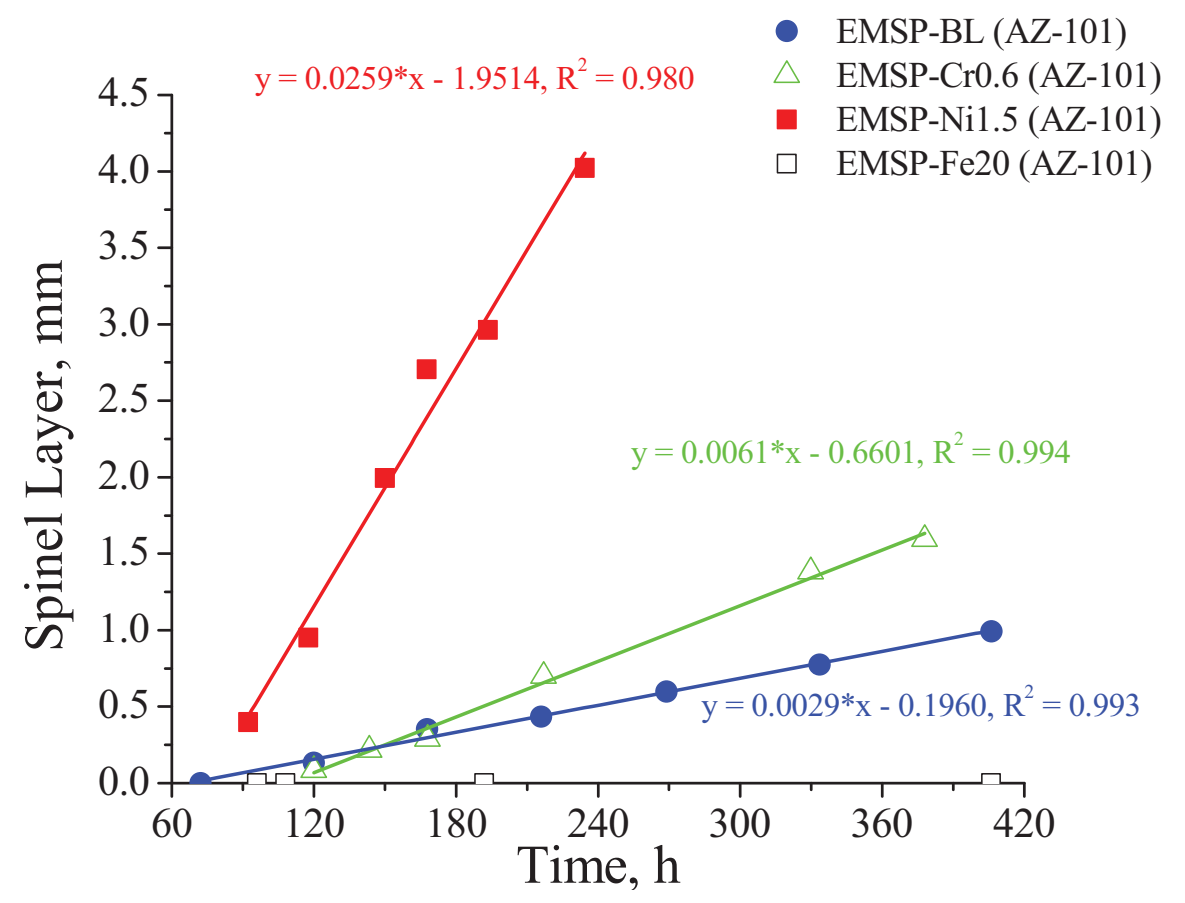

Figure 9.2. Thickness of Spinel Layer as a Function of Time for Selected EMSP (AZ-101) Glasses that Were Heat-Treated in Double Crucibles at $850^{\circ} \mathrm{C}$ 
A

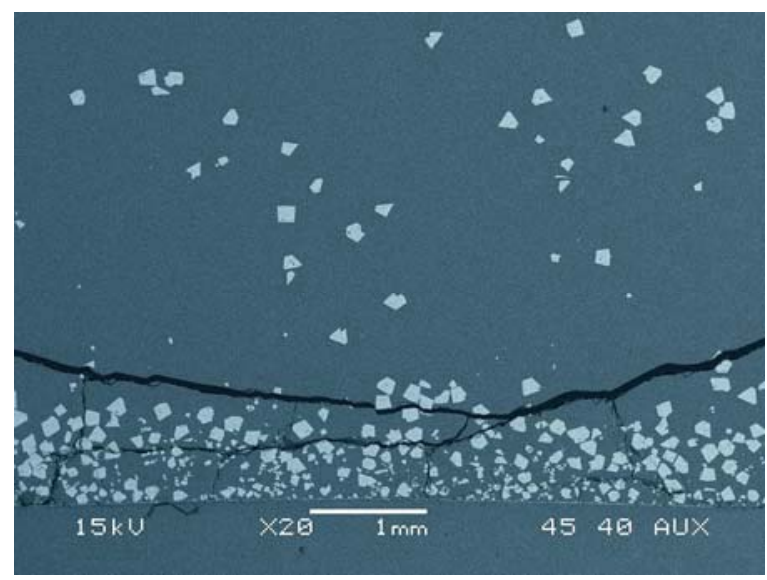

$\mathrm{C}$

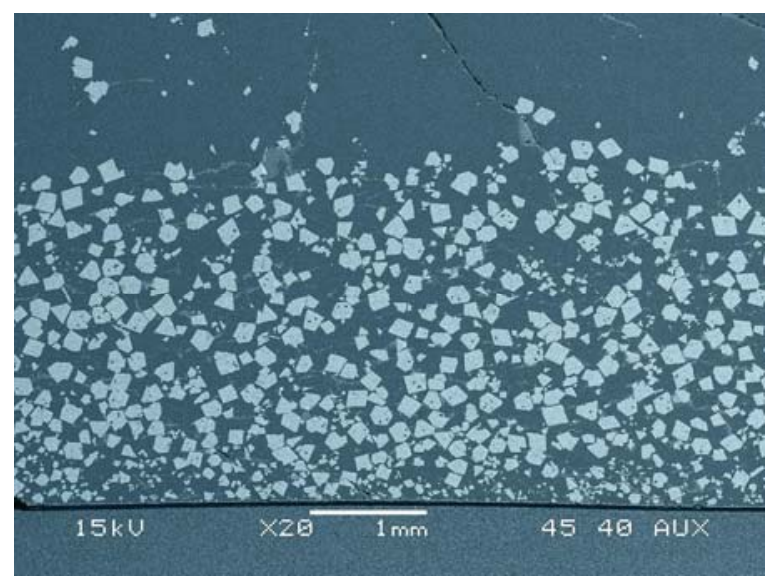

B

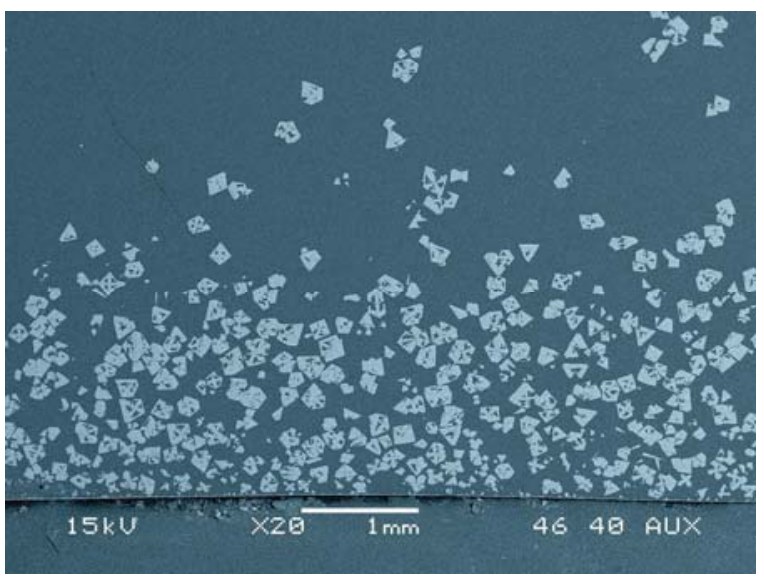

$\mathrm{D}$

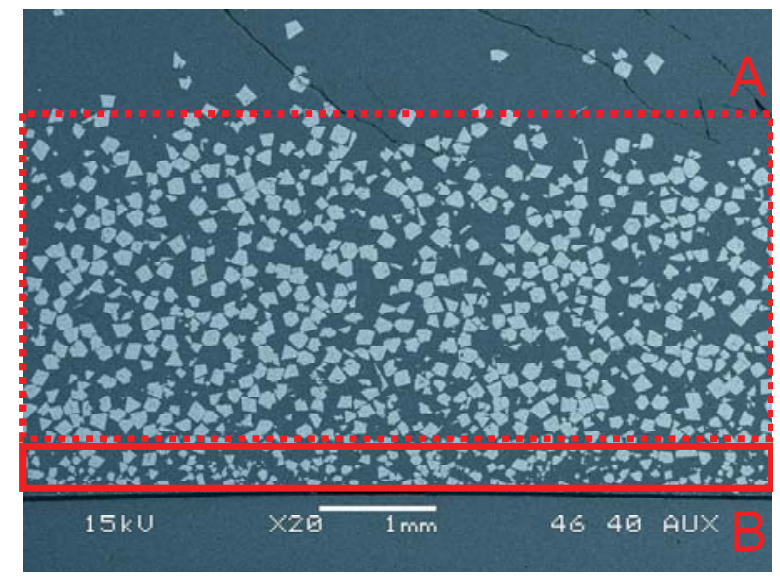

Figure 9.3. SEM Images of Spinel Layer in EMSP-Ni 1.5-(AZ-101) Glass After Heat-Treatment at $850^{\circ} \mathrm{C}$ for $92 \mathrm{~h} \mathrm{(A),} 150 \mathrm{~h}(\mathrm{~B}), 168 \mathrm{~h}(\mathrm{C})$, and $194 \mathrm{~h}(\mathrm{D})$

A

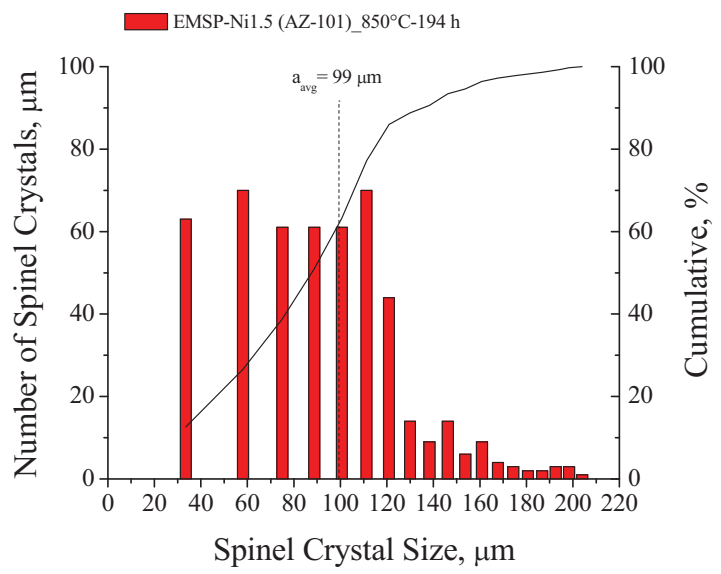

B

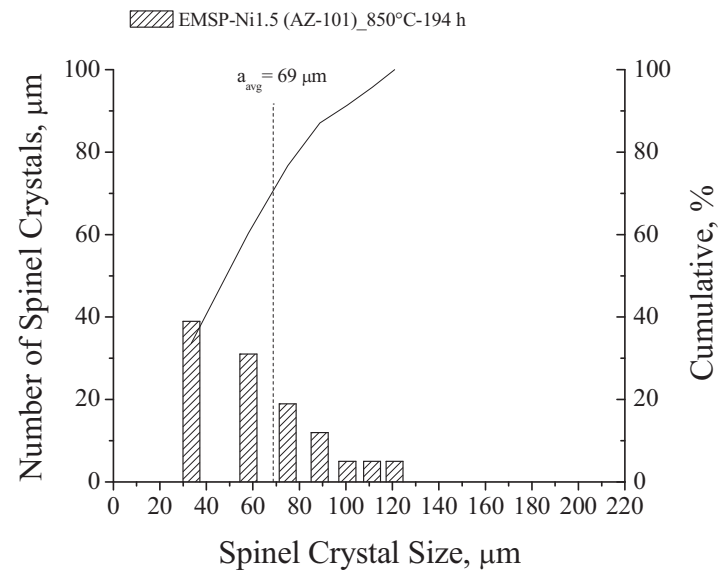

Figure 9.4. Distribution of Average Crystal Size in the Layer of Settled Spinel in EMSP-Ni 1.5(AZ-101) Glass After Heat-Treatment at $850^{\circ} \mathrm{C}$ for $194 \mathrm{~h}$ (Figure 9.3D): top $2.6 \mathrm{~mm}$ (A) and bottom $\sim 0.36 \mathrm{~mm}$ (B) 


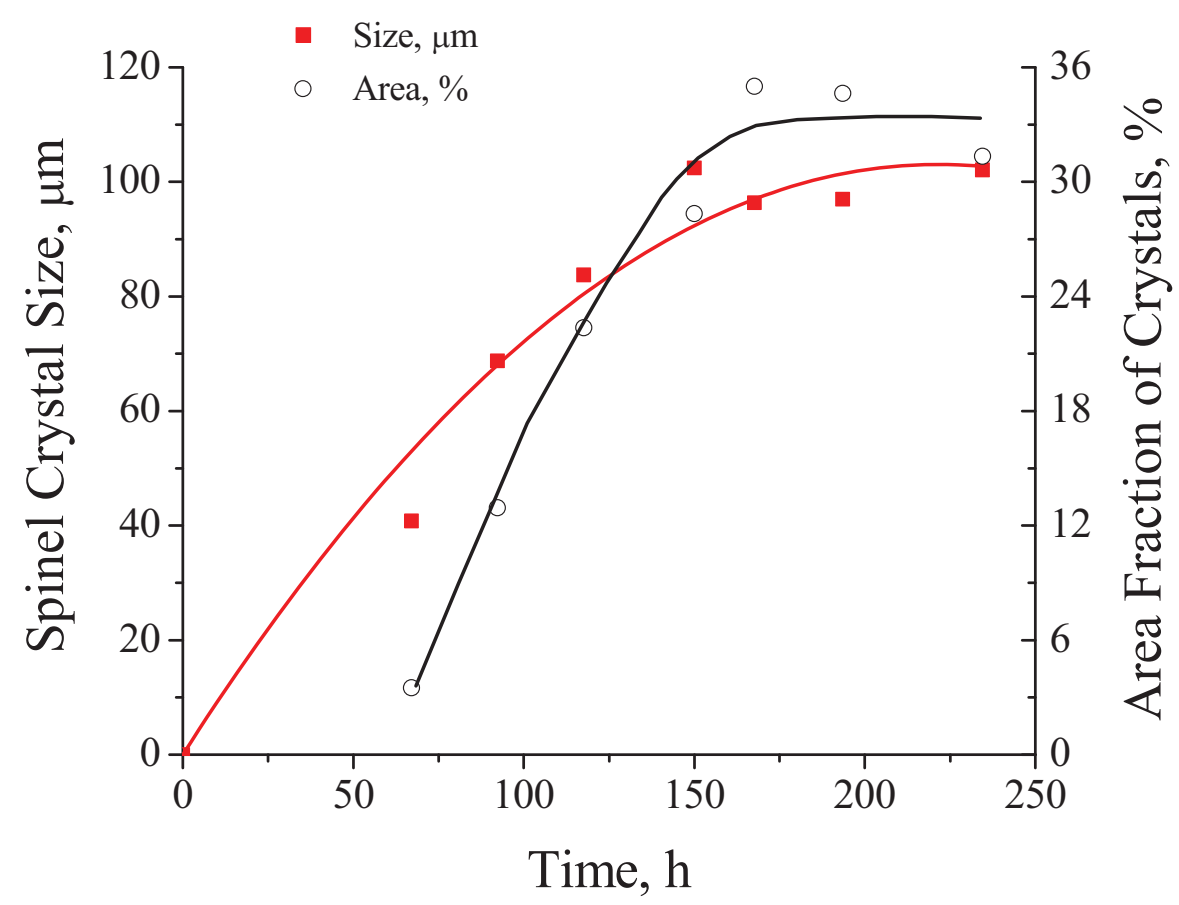

Figure 9.5. Change of Average Spinel Crystal Size and Layer Compaction over Time in EMSP-Ni 1.5(AZ-101) Glass Heat-Treated at $850^{\circ} \mathrm{C}$; the lines are there to help guide the eye

A question arises whether it would be possible to control the accumulation rate of spinel crystals by optimizing the concentration of noble metals in radioactive wastes. A small concentration of noble metals may be sufficient to nucleate enough spinel crystals to limit their growth to a size of approximately $10 \mu \mathrm{m}$. High concentrations of noble metals are not favorable because of their tendency to form large agglomerates. These agglomerates settle rapidly, regardless of phase equilibria. In contrast, the glass containing $20 \mathrm{wt} \%$ of $\mathrm{Fe}_{2} \mathrm{O}_{3}$ did not form the spinel layer even after 17 days at $850^{\circ} \mathrm{C}$ (Figure 9.2). Adding $\sim 5 \mathrm{wt} \%$ of $\mathrm{Fe}_{2} \mathrm{O}_{3}$ to the baseline glass significantly increased the number density of spinel crystals ( 700 to 850 crystals $/ \mathrm{mm}^{2}$ ) and decreased their size to $\sim 10 \mu \mathrm{m}$ on average (Figure 9.6). Figure 9.7 shows the thickness of the spinel layer as a function of time for three glasses that were heat-treated in high-chromia crucibles at $850^{\circ} \mathrm{C}$. The accumulation rate of spinel crystals in high-chromia crucibles was only slightly higher than that in double crucibles, except for baseline glass that was $\sim 2.6$ times faster. The enrichment of this glass with chromium that was leached out from the wall resulted in an increased number of $\sim 50-\mu \mathrm{m}$ chromium-enriched spinel crystals. Table 9.1 summarizes the concentration of $\mathrm{Cr}, \mathrm{Fe}$, and $\mathrm{Ni}$ in two glasses at the center of the cross-sectioned crucible $\sim 2 \mathrm{~mm}$ from the left (L) and the right (R) walls and in the middle (M). Glasses were heat-treated in high-chromia crucibles at $850^{\circ} \mathrm{C}$ for 4 days. Significantly higher concentrations of $\mathrm{Cr}_{2} \mathrm{O}_{3}$ were detected in areas close to the refractory wall because of the dissolution of high-chromia refractory by molten glasses. 


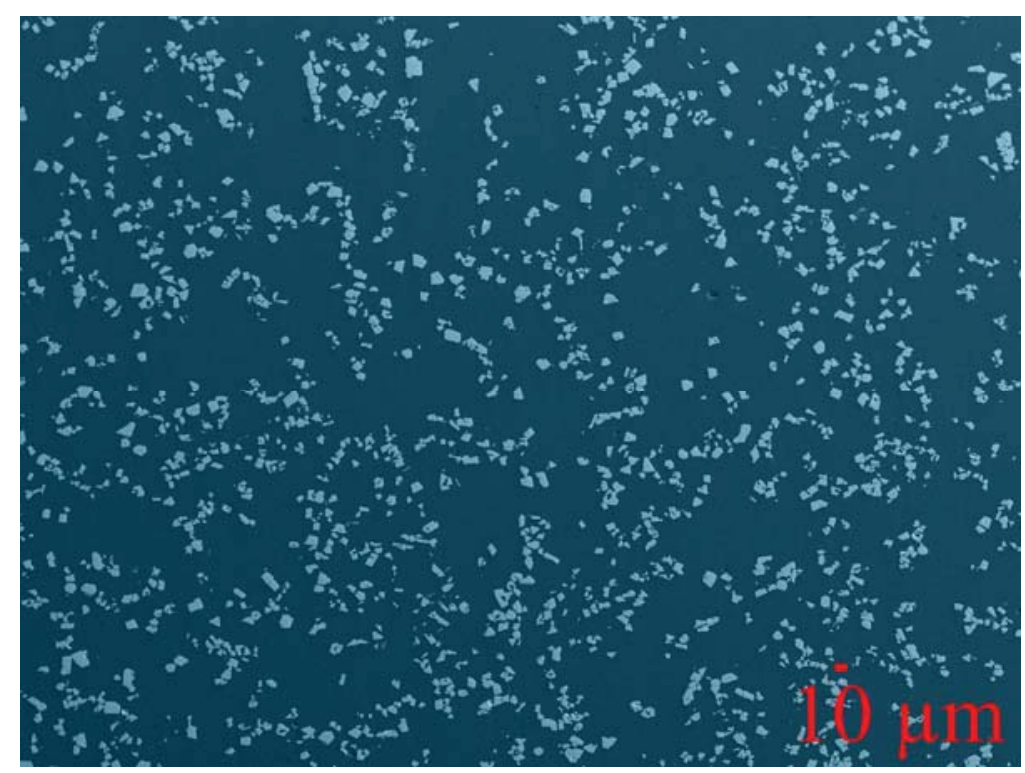

Figure 9.6. SEM Image of EMSP-Fe20-(AZ-101) Glass Heat-Treated at $850^{\circ} \mathrm{C}$ for 27 Days

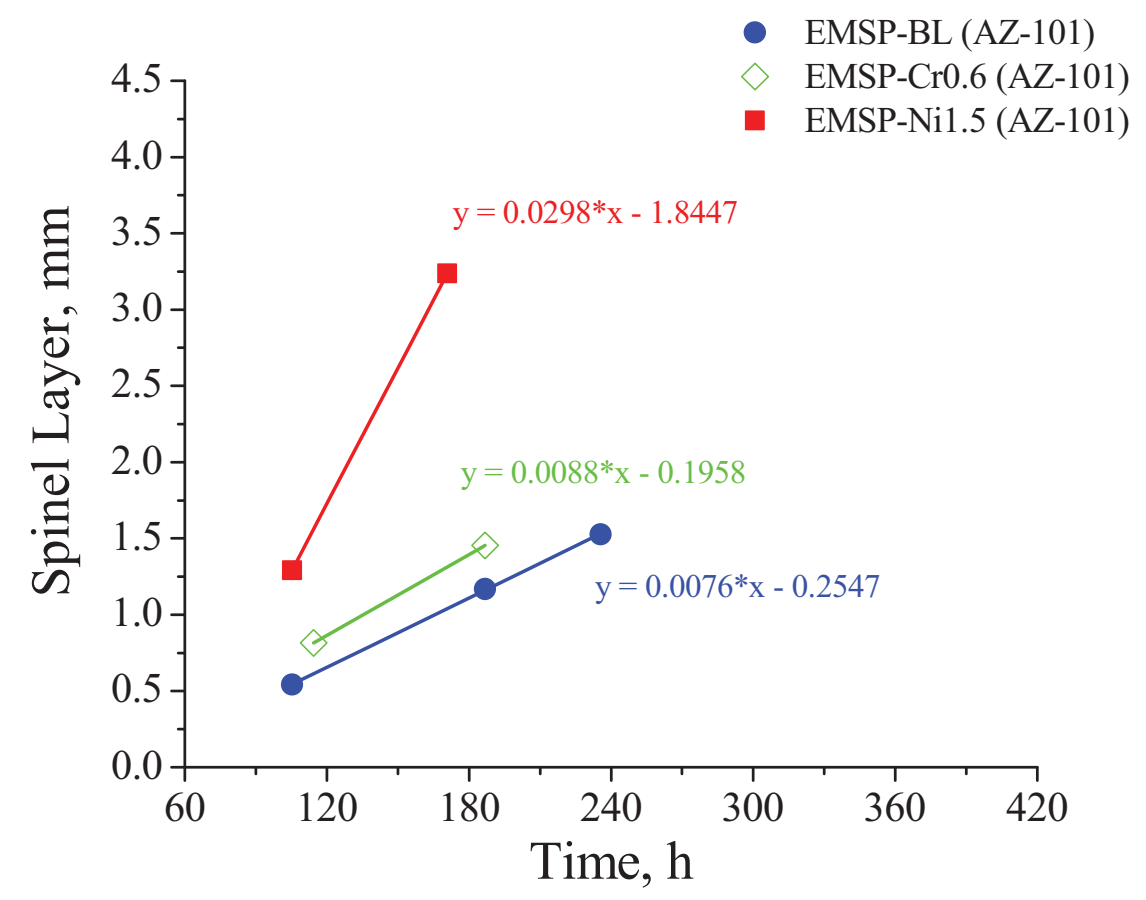

Figure 9.7. Thickness of Spinel Layer as a Function of Time for Selected EMSP (AZ-101) Glasses that Were Heat-Treated in High-Chromia Crucibles at $850^{\circ} \mathrm{C}$ 
Table 9.1. Concentration of $\mathrm{Cr}, \mathrm{Fe}$, and $\mathrm{Ni}$ in Glass in Mass \% of Oxides at the Center of the CrossSectioned Crucible $\sim 2 \mathrm{~mm}$ from the Left (L) and the Right (R) Walls and in the Middle (M). Glasses were Heat-Treated in High-Chromia Crucibles at $850^{\circ} \mathrm{C}$ for 4 Days.

\begin{tabular}{ccccc}
\hline Glass ID & Location & $\mathrm{Cr}_{2} \mathrm{O}_{3}$ & $\mathrm{Fe}_{2} \mathrm{O}_{3}$ & $\mathrm{NiO}$ \\
\hline \multirow{3}{*}{ EMSP-BL (AZ-101) } & Glass L & 0.56 & 3.32 & 0.05 \\
& Glass M & 0.18 & 10.7 & 0.44 \\
& Glass R & 1.29 & 8.48 & 0.17 \\
\hline \multirow{3}{*}{ EMSP-Ni1.5 (AZ-101 } & Glass L & 1.17 & 5.83 & 0.23 \\
& Glass M & 0.15 & 9.5 & 0.53 \\
& Glass R & 0.9 & 7.05 & 0.13 \\
\hline
\end{tabular}

Spinel, a solid solution of magnetite $\left(\mathrm{Fe}_{3} \mathrm{O}_{4}\right)$, trevorite $\left(\mathrm{NiFe}_{2} \mathrm{O}_{4}\right)$, and nichromite $\left(\mathrm{NiCr}_{2} \mathrm{O}_{4}\right)$, was the primary crystalline phase of designed EMSP glasses. The main factor that determined the accumulation rate of spinel crystals was the crystal size. Large crystals formed with an increased concentration of $\mathrm{NiO}$ and settled rapidly regardless of phase equilibria. A small concentration of noble metals in the molten glasses prevented the formation of large spinel crystals. An increased concentration of $\mathrm{Fe}_{2} \mathrm{O}_{3}$ resulted in a high number density of $\sim 10-\mu \mathrm{m}$ spinel crystals that remained suspended in the glass. The enrichment of glass with chromium resulted in an increased number of $\sim 50-\mu \mathrm{m}$, chromiumenriched spinel crystals.

A double crucible test was also performed with glasses prepared from chemicals. These glasses were formulated to validate the results obtained using AZ-101 glasses, and these results elucidate in more detail the effect of noble metals $\left(\mathrm{Rh}_{2} \mathrm{O}_{3}, \mathrm{RuO}_{2}\right), \mathrm{Al}_{2} \mathrm{O}_{3}$, and $\mathrm{Fe}_{2} \mathrm{O}_{3}$ on the size and number density of crystals as well as the thickness of the settled layer. Figure 9.8 shows the growth of the settling layer for Ni-rich glass, EMSP-Ni1.5-(Chem.), which is heat-treated in double crucibles at $850^{\circ} \mathrm{C}$ for different time periods. This glass was a replica of EMSP-Ni1.5/nm-(AZ-101), including the same concentration of $\mathrm{Rh}_{2} \mathrm{O}_{3}$ and $\mathrm{RuO}_{2}$ but prepared from chemicals. They both show the same trend. Adding noble metals to Ni-rich glasses decreased the size of crystals and increased their number density, and therefore, decreased the settling rate of crystals and the growth of the settling layer. The crystals in Ni-reach (Chem.) glass were even smaller after $\mathrm{RuO}_{2}$ was added to achieve total concentrations of 0.029 and $0.087 \mathrm{wt} \%$ in the following glasses: EMSP-Ni1.5/0.029 $\mathrm{RuO}_{2}$-(Chem.) and EMSP-Ni1.5/0.087 $\mathrm{RuO}_{2}$-(Chem.). Table 9.2 summarizes the changes in crystal size, number density, and thickness of settled layers as a function of time for these glasses.

Table 9.3 summarizes the effect of higher concentrations of alumina and iron on crystal sizes and number densities as well as the thicknesses of the settled layers in Ni-rich glasses. Both components quite efficiently suppressed the formation of settled layers by decreasing the crystal size below or around $10 \mu \mathrm{m}$ and increasing the number density of crystals by more than 1000 crystals per $\mathrm{mm}^{2}$. The high number of small crystals can be explained by the presence of hematite and nepheline, which act as nucleating agents of spinel crystals. In spite of the good performance of alumina, the precipitation of nepheline that was observed in the Al-rich glasses (Figure 9.9) cannot be tolerated because nepheline decreases the durability of the glass waste form. In contrast, no problems were detected for Fe-rich glasses. This suggests that the mixing of waste streams containing high concentrations of Fe with quite dangerous Ni-enriched streams can restrain or even stop the formation of the settling layer in the riser during melter idling. 


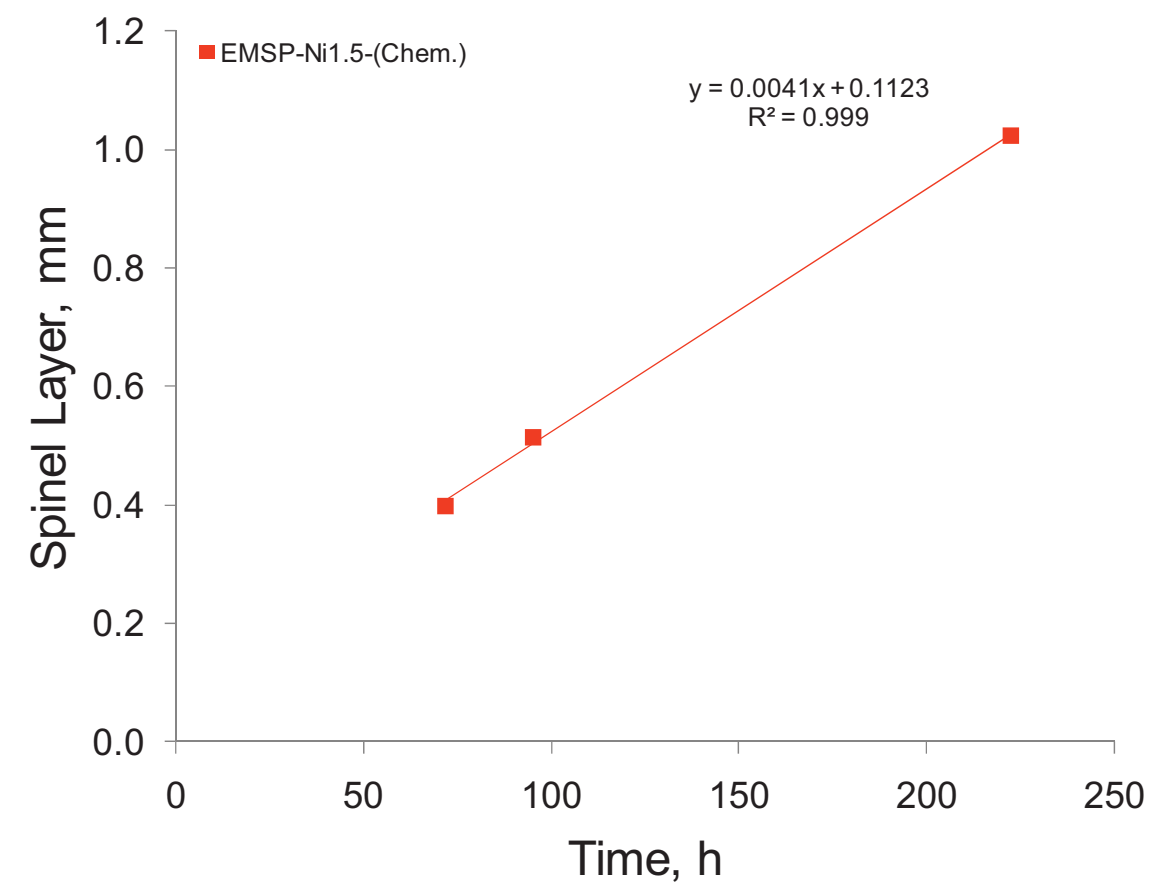

Figure 9.8. Thickness of Settled Layer as a Function of Time for Selected EMSP-Ni1.5-(Chem.) Glass Heat-Treated in Double Crucibles at $850^{\circ} \mathrm{C}$

Table 9.2. The Effect of $\mathrm{Ru}_{2} \mathrm{O}_{3}$ on Thickness of Settled Layers, Crystal Sizes, and Crystal Number Densities for Ni-rich (Chem) Glasses

\begin{tabular}{lcccc}
\hline \multirow{2}{*}{ Glass } & $\begin{array}{c}\text { Time, } \\
\mathrm{h}\end{array}$ & $\begin{array}{c}\text { Settled layer, } \\
\mu \mathrm{m}\end{array}$ & $\begin{array}{c}\text { Crystal size, } \\
\mu \mathrm{m}\end{array}$ & $\begin{array}{c}\text { Number density, } \\
\text { number of crystals } / \mathrm{mm}^{2}\end{array}$ \\
\hline EMSP-Ni1.5-(Chem.) & 72.0 & 399.1 & 19.5 & 71 \\
& 95.4 & 515.3 & 36.6 & 16 \\
& 222.3 & 1024.5 & 33.3 & 23 \\
\hline EMSP-Ni1.5/0.029 $\mathrm{RuO}_{2}$-(Chem.) & 168.3 & 0.0 & 5.9 & 786 \\
& 309.8 & 172.3 & 10.0 & 356 \\
\hline EMSP-Ni1.5/0.087 $\mathrm{RuO}_{2}$-(Chem.) & 96.0 & 0.0 & 4.7 & 1449 \\
& 168.5 & 0.0 & 4.7 & 1108 \\
& 313.0 & 0.0 & 5.0 & 1475 \\
\hline
\end{tabular}


Table 9.3. The Effect of $\mathrm{Al}_{2} \mathrm{O}_{3}$ and $\mathrm{Fe}_{2} \mathrm{O}_{3}$ on Thickness of Settled Layers, Crystal Sizes, and Crystal Number Densities for Ni-rich (Chem) Glasses

\begin{tabular}{lcccc}
\hline Glass & $\begin{array}{c}\text { Time, } \\
\mathrm{h}\end{array}$ & $\begin{array}{c}\text { Settled layer, } \\
\mu \mathrm{m}\end{array}$ & $\begin{array}{c}\text { Crystal size, } \\
\mu \mathrm{m}\end{array}$ & $\begin{array}{c}\text { Number density, } \\
\text { number of crystals/mm }\end{array}$ \\
\hline EMSP-Ni1.5-(Chem.) & 72.0 & 399.1 & 19.5 & 71 \\
& 95.4 & 515.3 & 36.6 & 16 \\
& 222.3 & 1024.5 & 33.3 & 23 \\
\hline EMSP-Ni1.5/A112-(Chem.) & 120.1 & 0.0 & 5.1 & 1061 \\
& 199.6 & 0.0 & 4.9 & 1400 \\
& 309.8 & 0.0 & 5.1 & 1314 \\
\hline EMSP-Fe20/Ni1.5-(Chem.) & 237.7 & 0.0 & 10.1 & 1250 \\
\hline
\end{tabular}

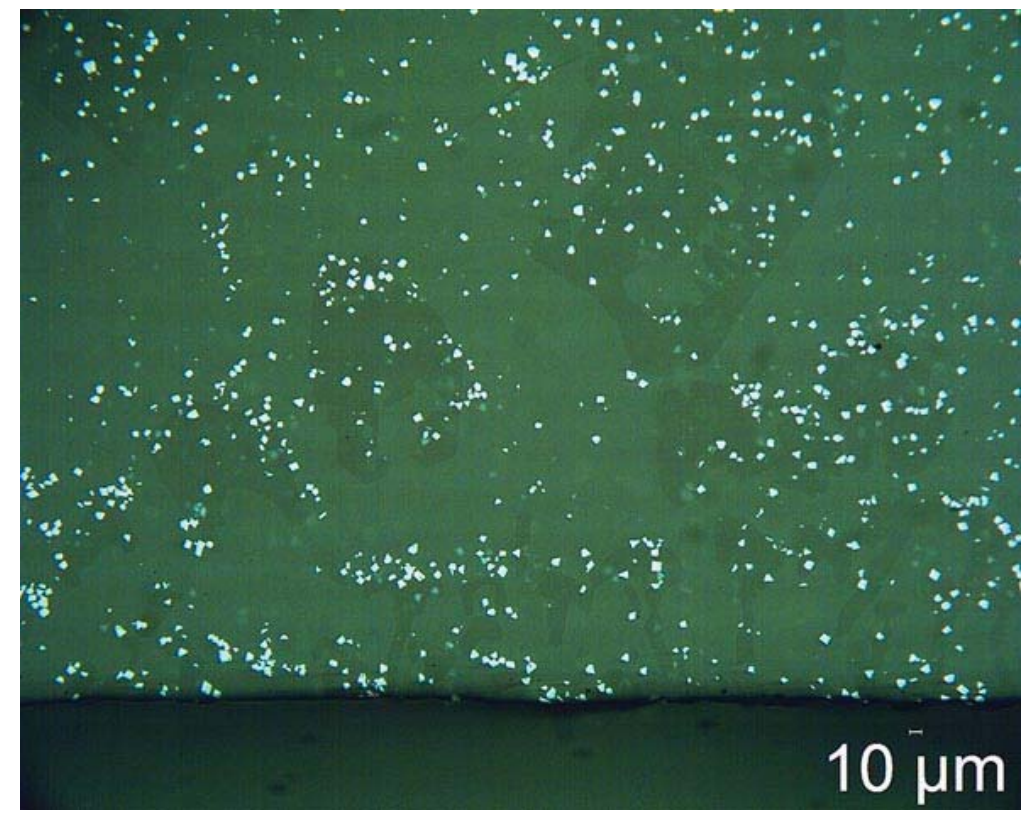

Figure 9.9. EMSP-Ni1.5/A112-(Chem.) Glass After $\sim 310 \mathrm{~h}$ at $850^{\circ} \mathrm{C}$ in Double Crucible; White Features-Spinel Crystals, Dark Features-Nepheline

Table 9.4 summarizes the effect of noble metals, iron, chromium, and manganese on crystal sizes and number densities as well as the thicknesses of the settled layers for other tested glasses prepared from chemicals. The collected experimental data confirmed the positive effect of noble metals and iron on suppressing the formation of spinel sludge layer. The effect of spinel formers other than Ni on settling later was not as dramatic. They did not increase the size of crystals to more than $\sim 19 \mu \mathrm{m}$ and did not even double the thickness of the layer after 10 days at $850^{\circ} \mathbf{C}$. 
Table 9.4. The Effect of $\mathrm{Al}_{2} \mathrm{O}_{3}$ and $\mathrm{Fe}_{2} \mathrm{O}_{3}$ on Thickness of Settled Layers, Crystal Sizes, and Crystal Number Densities for Glasses Prepared from Chemicals

\begin{tabular}{lcccc}
\hline \multirow{2}{*}{ Glass } & Time, $\mathrm{h}$ & $\begin{array}{c}\text { Settled } \\
\text { Layer, } \mu \mathrm{m}\end{array}$ & $\begin{array}{c}\text { Crystal size, } \\
\mu \mathrm{m}\end{array}$ & $\begin{array}{c}\text { Number density, } \\
\# / \mathrm{mm}^{2}\end{array}$ \\
\hline EMSP-BL-(Chem.) & 120.0 & 0.0 & 14.4 & 65 \\
& 168.0 & 0.0 & 9.1 & 202 \\
& 242.0 & 178.8 & 18.5 & 97 \\
\hline EMSP-BL/w/o nm-(Chem.) & 120.0 & 0.0 & 57.1 & 2 \\
\hline EMSP-Fe20-(Chem.) & 24.0 & 0.0 & 3.5 & 2158 \\
\hline EMSP-Cr0.6-(Chem.) & 96.0 & 0.0 & 4.2 & 781 \\
& 168.0 & 0.0 & 4.4 & 392 \\
\hline EMSP-Mn1-(Chem.) & 242.0 & 269.4 & 9.0 & 249 \\
& 96.5 & 0.0 & 9.3 & 277 \\
\hline EMSP-Mn2.5-(Chem.) & 188.0 & 0.0 & 9.4 & 248 \\
\hline & 264.3 & 70.8 & 9.9 & 301 \\
& 102.2 & 0.0 & 8.9 & 381 \\
\hline
\end{tabular}





\subsection{Empirical Model of Spinel Crystal Settling}

Three stages were identified during the settling experiments in the double crucibles: 1) latency period with no settling, 2) settling period with constant settling rate of spinel, and 3) end of settling period with a low and gradually decreasing settling rate of spinel due to a smaller and smaller number of settling crystals. Only the sludge layer thickness data for AZ-101 glasses that were collected during the constant settling rate period were used to build an experimental model predicting crystal accumulation in the glass discharge riser as a function of seven major components $\left(\mathrm{Al}_{2} \mathrm{O}_{3}, \mathrm{Cr}_{2} \mathrm{O}_{3}, \mathrm{Fe}_{2} \mathrm{O}_{3}, \mathrm{ZnO}, \mathrm{MnO}, \mathrm{NiO}\right.$, and Others). The constant settling rate allowed us to use a general linear model in the form:

$$
h=\sum_{i=1}^{7} h_{i} x_{i}+t \sum_{i=1}^{7} s_{i} x_{i}
$$

where $h_{i}$ is a compositional dependent intercept coefficient $(\mu \mathrm{m}), t$ is the settling time $(\mathrm{h})$, and $s_{i}$ is a compositional dependent velocity coefficient $(\mu \mathrm{m} / \mathrm{h})$. Table 10.1 shows the calculated coefficients $h_{i}$ and $s_{i}$, the values of $\mathrm{R}^{2}$ (expresses the fraction of the variability accounted for by the model), and $R_{a d j}^{2}$ (adjust $\mathrm{R}^{2}$ for the number of parameters used in fitting the model). Negative coefficients $s_{i}$ for $\mathrm{Al}_{2} \mathrm{O}_{3}$ and $\mathrm{Fe}_{2} \mathrm{O}_{3}$ suggest that these components decrease the settling rate of crystals. In contrast, additions of $\mathrm{MnO}, \mathrm{ZnO}$, $\mathrm{Cr}_{2} \mathrm{O}_{3}$, and $\mathrm{NiO}$ to the baseline glass increase the settling rate. From this group, $\mathrm{NiO}$ stands out as the most troublesome component with a more than six times faster settling rate than, for example, $\mathrm{Cr}$-rich glass. The experiments showed that the detrimental effect of this component on the settling rate can be significantly suppressed by introducing the noble metals or $\mathrm{Fe}_{2} \mathrm{O}_{3}$ to the glass. The negative coefficients $h_{i}$ for $\mathrm{MnO}, \mathrm{ZnO}, \mathrm{Cr}_{2} \mathrm{O}_{3}$, and $\mathrm{NiO}$ only indicate, but do not predict, the length of the latency period. This period is dependent on the initial growth rate of crystals to the size at which crystals start to settle. Figure 10.1 shows the predictive versus measured thicknesses of a spinel sludge layer for AZ-101 glasses. The linear empirical model with coefficients $h_{i}$ and $s_{i}$ expressed as a linear function of mass fractions of seven major components fits the 35 data points reasonably well, $\mathrm{R}^{2}=0.985$, and can become an efficient tool to formulate the crystal-tolerant glasses that would ultimately allow a substantial increase in the waste loading.

Table 10.1. Component Coefficients Calculated with PNNL Model

\begin{tabular}{llc}
\hline Components & $h_{i}(\mu \mathrm{m})$ & $s_{i}(\mu \mathrm{m} / \mathrm{h})$ \\
\hline $\mathrm{Al}_{2} \mathrm{O}_{3}$ & 8816.97 & -350.41 \\
$\mathrm{Fe}_{2} \mathrm{O}_{3}$ & 4304.182 & -49.9117 \\
$\mathrm{MnO}$ & -7498.52 & 259.3812 \\
$\mathrm{ZnO}$ & -12257.6 & 313.0436 \\
$\mathrm{Cr}_{2} \mathrm{O}_{3}$ & -40257.3 & 443.5807 \\
$\mathrm{NiO}$ & -197477 & 2672.734 \\
Others & -366.91 & 27.00287 \\
$\mathrm{R}^{2}$ & \multicolumn{2}{c}{0.985} \\
$\mathrm{R}^{2}$ adj & \multicolumn{2}{c}{0.975} \\
\hline
\end{tabular}




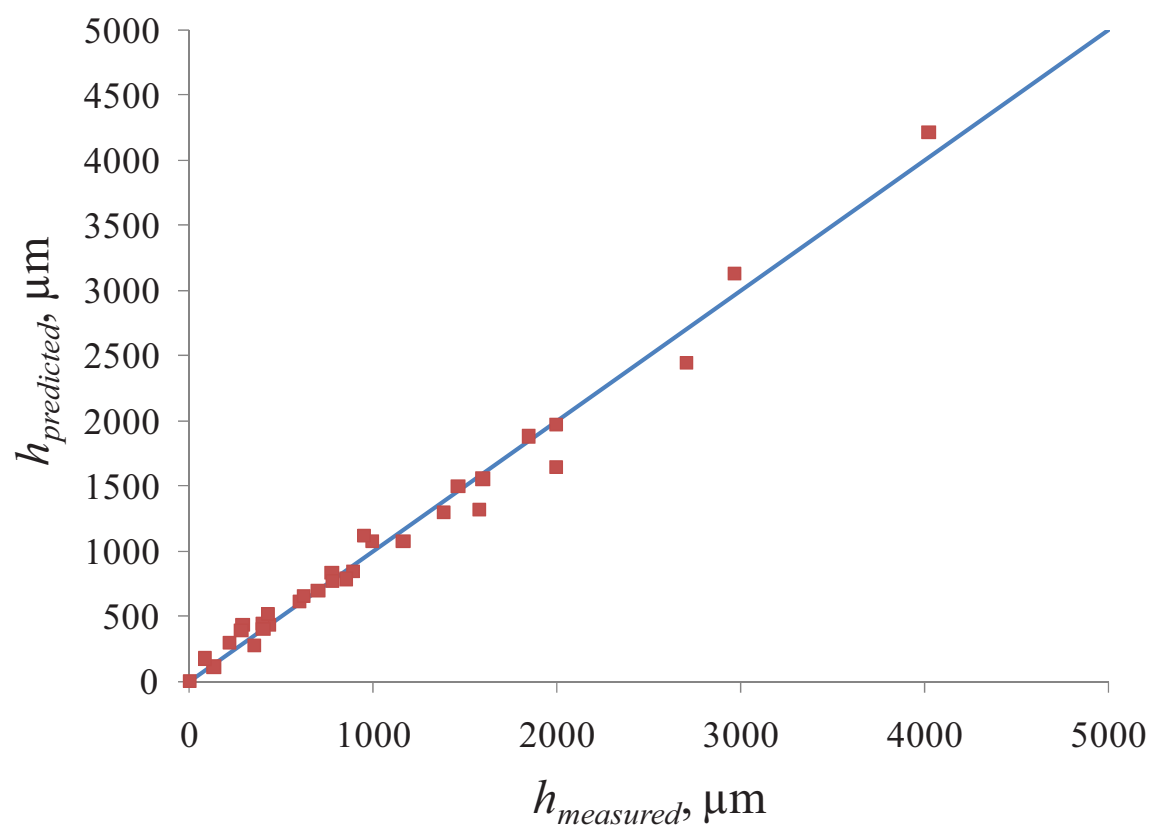

Figure 10.1. Predicted vs. Measured Spinel Layer Thickness 


\subsection{Physical Modeling of Particle Settling}

A major concern of the vitrification process is the formation and rapid settling of large spinel crystals in the glass discharge riser of the HLW melter during numerous and long melter idling periods from 20 to 100 days. For volume fractions in excess of 0.01 , the settling velocities of spinel crystals are reduced (hindered settling effect). To assess the crystal accumulation during melter operation, a detailed knowledge of spinel settling behavior in high-viscosity Newtonian liquids is needed. To determine the shape factor of single particles and the hindered settling function in binary mixtures, the settling of single particles and the motion of the settling front (for different volume fractions of solid) have been investigated through physical modeling.

\subsection{Free Settling}

The settling velocity of spherical particles suspended in a stagnant Newtonian fluid can be estimated from the well-known Stokes equation:

$$
v=\frac{2\left(\rho_{p}-\rho_{f}\right) g r^{2}}{9 \eta}
$$

where $\rho_{p}$ is the particle density, $\rho_{f}$ is the fluid density, $\eta$ is the fluid dynamic viscosity, $g$ is the gravitational acceleration, and $r$ is the particle effective radius. This equation can be modified by considering non-spherical particles and particle-fluid-particle interactions in concentrated suspensions:

$$
v=k_{s} k_{h} \frac{\left(\rho_{p}-\rho_{f}\right) g r^{2}}{\eta}
$$

where $k_{s}$ is the Stokes shape factor, and $k_{h}$ is the hindered settling coefficient. The shape factor of the non-spherical particle in diluted suspensions $\left(k_{h}=0\right)$ can be calculated from experimentally measured terminal settling velocities using the equation:

$$
k_{s}=\frac{v^{\prime}}{v}=\frac{v^{\prime} \eta}{\left(\rho_{p}-\rho_{f}\right) g r_{v}^{2}}
$$

where $v^{\prime}$ is the experimentally measured terminal settling velocity, and $r_{v}$ is the particle effective radius.

Settling experiments were performed with spherical glass beads from the Mo-Sci Corporation and octahedral spinel crystals that were suspended in Brookfield 0.48, 0.98, 1.025, 4.84, and 5.08 Pa.s standard viscosity oils of specific gravity $0.97 \mathrm{~g} / \mathrm{cm}^{3}$. These low viscosities (lower than that of the glass at $850^{\circ} \mathrm{C}$ ) allowed the measurements to be performed in a short time and should not affect the hindered settling function. The barium calcium silicate glass beads had a density $4.1741 \mathrm{~g} / \mathrm{cm}^{3}$, and the average diameter was 72 and $161 \mu \mathrm{m}$. The soda-lime-silica glass beads had a density $2.4842 \mathrm{~g} / \mathrm{cm}^{3}$, and the average diameter was 67 and $137 \mu \mathrm{m}$. The spinel crystals were produced by precipitation in the waste simulant glass during 10 days of long heat-treatment at $850^{\circ} \mathrm{C}$. The glass was dissolved overnight in continuously mixed $25 \% \mathrm{HNO}_{3}$ solution at $60^{\circ} \mathrm{C}$. The collected mixture of crystals and silica gel was 
then treated with 5\% HF to obtain the gel-free crystals that were separated into distinct size groups with sieving. The crystals have a density $5.3954 \mathrm{~g} / \mathrm{cm}^{3}$, as-measured with the gas pycnometer, and contained about 31.4 mass $\%$ of $\mathrm{Ni}, 48.3$ mass $\%$ of $\mathrm{Fe}, 1.3$ mass $\%$ of $\mathrm{Cr}$, and 19 mass $\%$ of $\mathrm{O}$, as-determined with scanning electron microscopy and energy dispersive spectroscopy (SEM-EDS). Figure 11.1 and Figure 11.2 show the shape and size distribution of tested glass beads and spinel crystals.

A

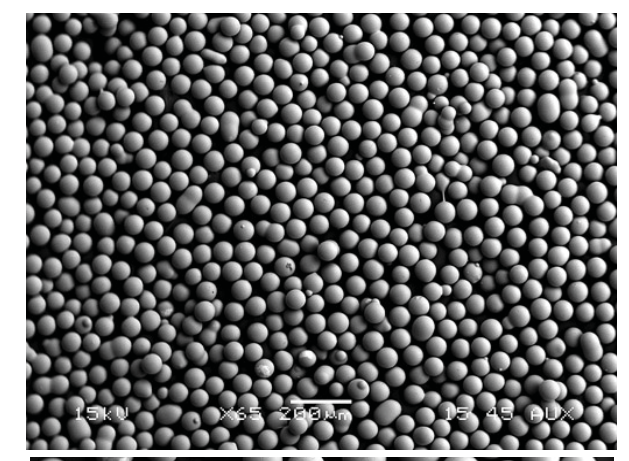

B

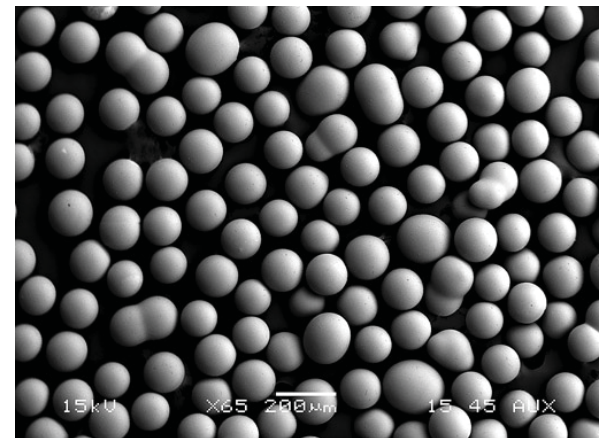

$\mathrm{C}$

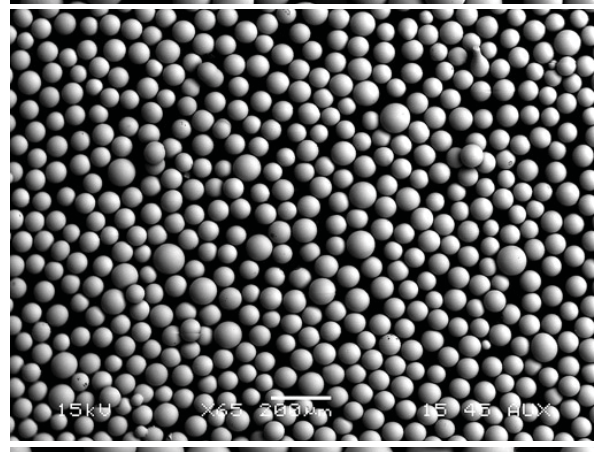

$\mathrm{D}$

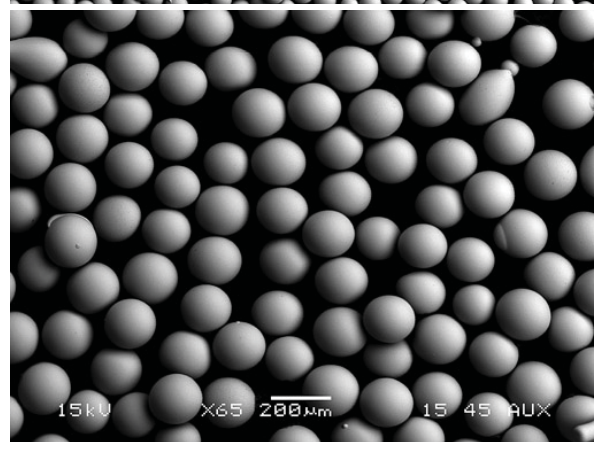

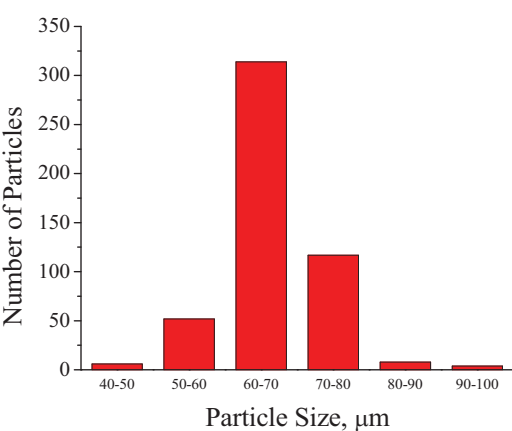
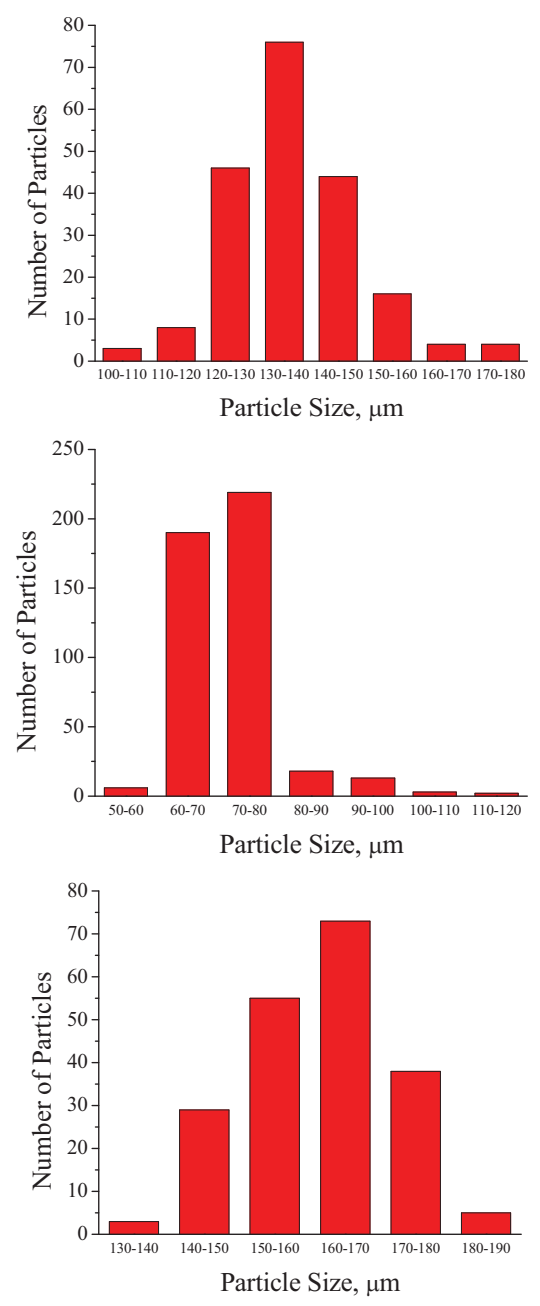

Figure 11.1. SEM images of Spherical Soda-Lime-Silica (A, B) and Ba-Ca silicate (C, D) Glass Beads and Their Particle Size Distribution
$\left(\mathrm{A}-\rho \sim 2.5 \mathrm{~g} / \mathrm{cm}^{3}, D_{p} \sim 67 \mu \mathrm{m} ; \mathrm{B}-\rho \sim 2.5 \mathrm{~g} / \mathrm{cm}^{3}, D_{p} 137 \mu \mathrm{m}\right.$;
$\left.\mathrm{C}-\rho \sim 4.2 \mathrm{~g} / \mathrm{cm}^{3}, D_{p} \sim 72 \mu \mathrm{m} ; \mathrm{D}-\rho \sim 4.2 \mathrm{~g} / \mathrm{cm}^{3}, D_{p} \sim 161 \mu \mathrm{m}\right)$. 

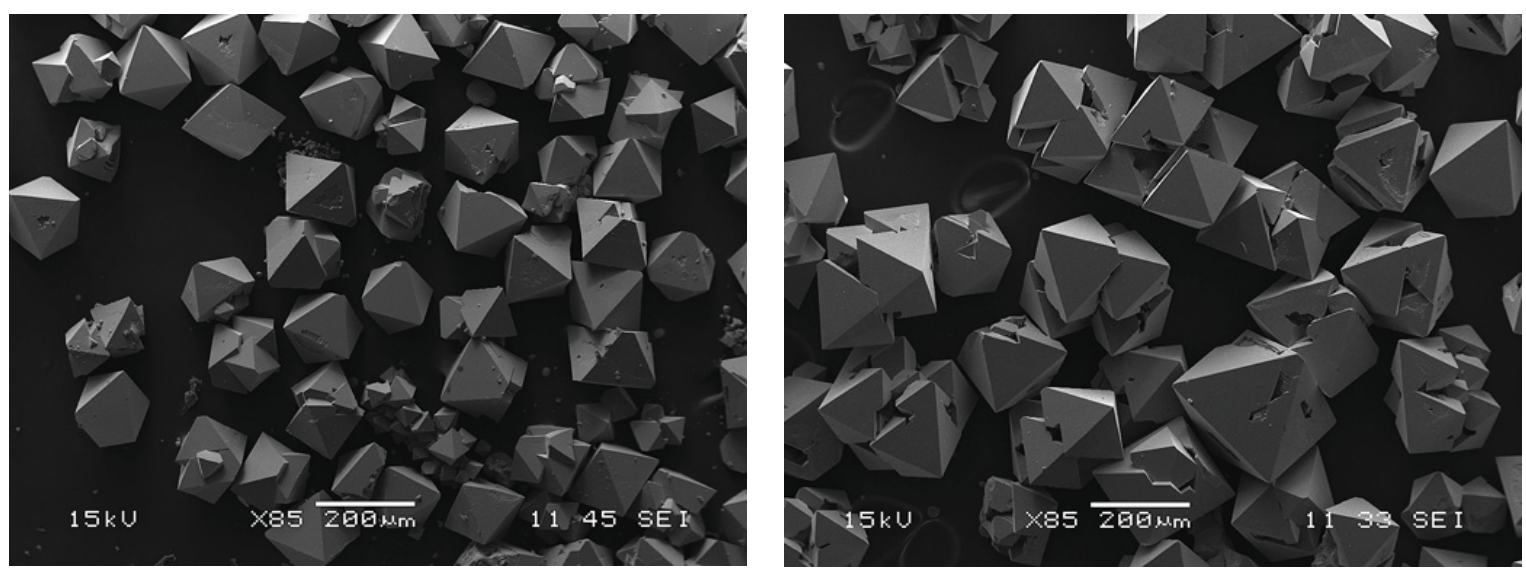

Figure 11.2. SEM Images of Octahedral Spinel Crystals

Figure 11.3 shows the experimental setup of the optical particle-dynamics analyzer for measuring the free-settling velocity of single particles and agglomerates. The setup consisted of 1 ) a square quartz cuvette (15 cm high, $2 \mathrm{~cm}$ deep, and $2 \mathrm{~cm}$ wide) filled with silicone oil, 2) a focus stage assembly, 3 ) an infinity-corrected long working distance lens $20 \times / 0.42$ with a rectangular field of view $415 \times 515 \mu \mathrm{m}$ (distance between adjacent lines was $10 \mu \mathrm{m}$ ) that was mounted on a digital camera and focused at the center of the cuvette approximately $4 \mathrm{~cm}$ above the bottom, and 4) a Paxcam charged coupled device (CCD) camera with illumination provided by a fiber optic illuminator equipped with sets of filters to achieve optimum contrast. All of the equipment was sitting on a heavy marble table to eliminate any vibration causing disturbance to the cuvette and associated optics.

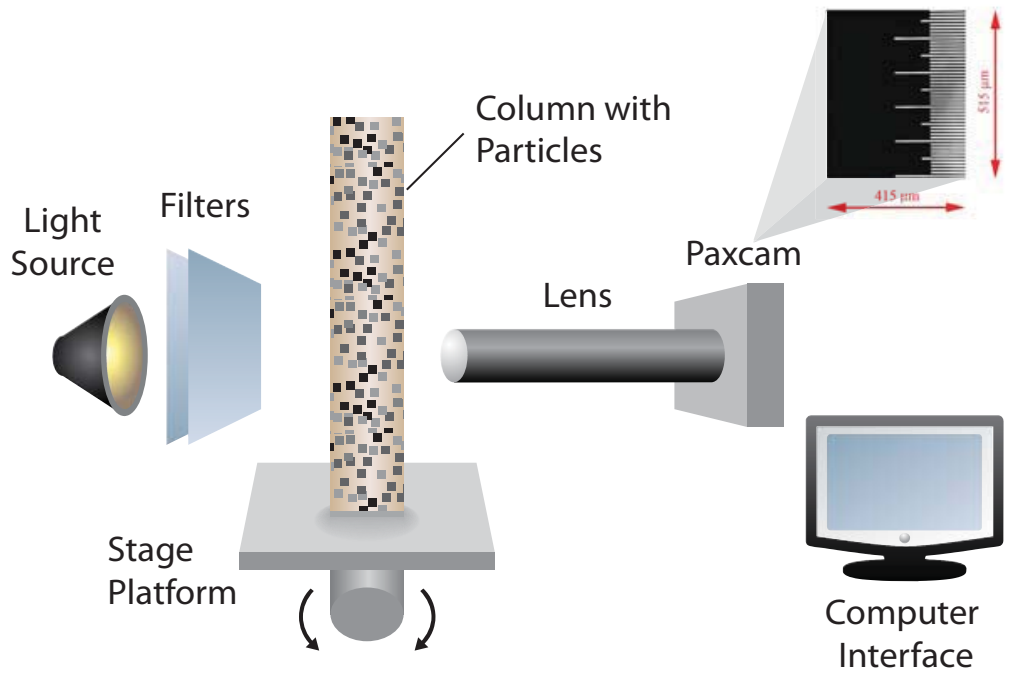

Figure 11.3. Optical Particle-Dynamics-Analyzer

The optical system was capable of measuring particle sizes in the range of 2 to $415 \mu \mathrm{m}$. The maximum terminal settling velocity that can be measured was $129 \mu \mathrm{m} / \mathrm{s}$.

To obtain terminal settling velocities of individual beads and crystals, a few particles were sprinkled with a sieve onto the surface of viscosity oil in a cuvette. The cuvette was tall enough for particles to reach their terminal settling velocities and wide enough that the wall effect on free-settling rates could be 
neglected. PAX-it version 7 imaging software was used to record images of settling particles at 5-second intervals and measure their size and vertical distances. Figure 11.4 shows an example of time-sequence images collected during the settling of spinel crystal, skipping every second image. The settling velocity was calculated by dividing the difference in distance between successive images by the known time interval. While the effective radius of the glass beads was measured directly from collected images, the non-spherical shape of the spinel crystals required the radius to be calculated for an equivalent volume sphere. Therefore, the average length of the crystal edge was determined to be $a=\left(L_{1}+L_{2}\right) / 2$, and the volume of an isometric octahedron was calculated from the equation:

$$
V_{s}=1 / 3 \sqrt{2} a^{3}
$$

Then, the equivalent sphere radius was acquired from the equation:

$$
r_{e q}=\left(\frac{3 V_{s}}{4 \pi}\right)^{1 / 3}
$$

In the case of agglomerates, the radius of an equivalent area was obtained from the measured boundary area of the agglomerates, $\mathrm{A}_{\mathrm{ag}}$, by calculating the radius of a sphere with an identical cross-sectional area:

$$
r_{e q}=\sqrt{\frac{A_{a g}}{\pi}}
$$

To determine the Stokes shape factors, the measured terminal settling velocities were plotted against $\left[\left(\rho_{p}-\rho_{f}\right) g R^{2}\right] / \eta$. The slopes of the linear regression curves corresponded to the unknown Stokes shape factors.
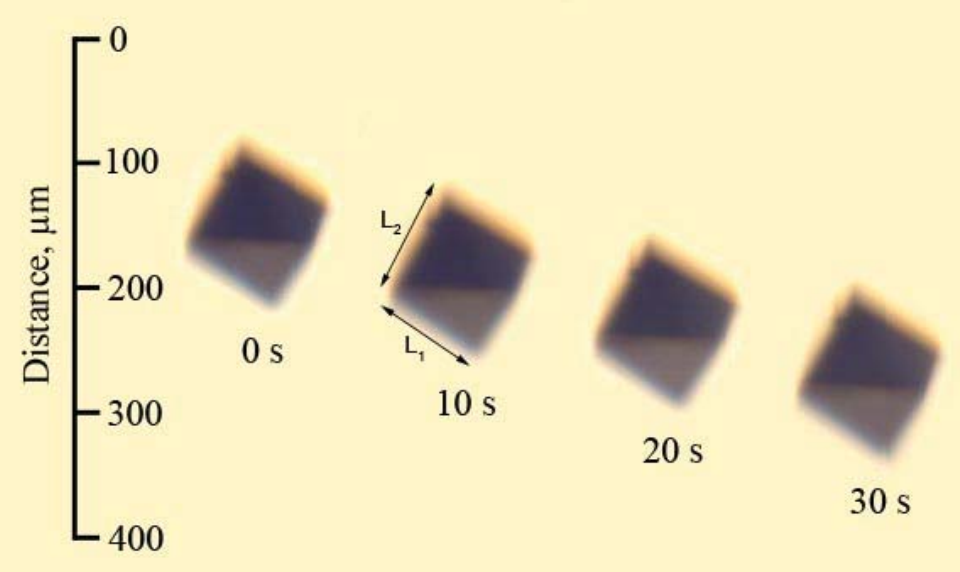

$30 \mathrm{~s}$

Figure 11.4. Time Sequence Images for Spinel with Edge Lengths $69 \mu \mathrm{m}\left(\mathrm{L}_{1}\right)$ and $79 \mu \mathrm{m}\left(\mathrm{L}_{2}\right)$ 
The preliminary settling experiments were performed with individual glass beads to evaluate our experimental method for determining particle shape factors. Table 11.1 shows the calculated settling velocities and shape factors for glass beads of different size and density in oils of three different viscosities. The shape factor was about constant, with an average value of 0.2219 and a relative standard deviation (RSD) of $\sim 1.5 \%$. Figure 11.5 shows a linear fit of settling velocity vs. $\left[\left(\rho_{p}-\rho_{f}\right) g R^{2}\right] / \eta$ for glass beads. A shape factor of 0.2227 from the fitting was almost identical to the tabulated Stokes shape factor for a perfect sphere, 2/9. The excellent agreement in shape factors indicated that a developed optical particle-dynamics analyzer can be efficiently used to study the settling of particles in the Stokes Regime $(\operatorname{Re}<0.5)$ and to determine their shape factors.

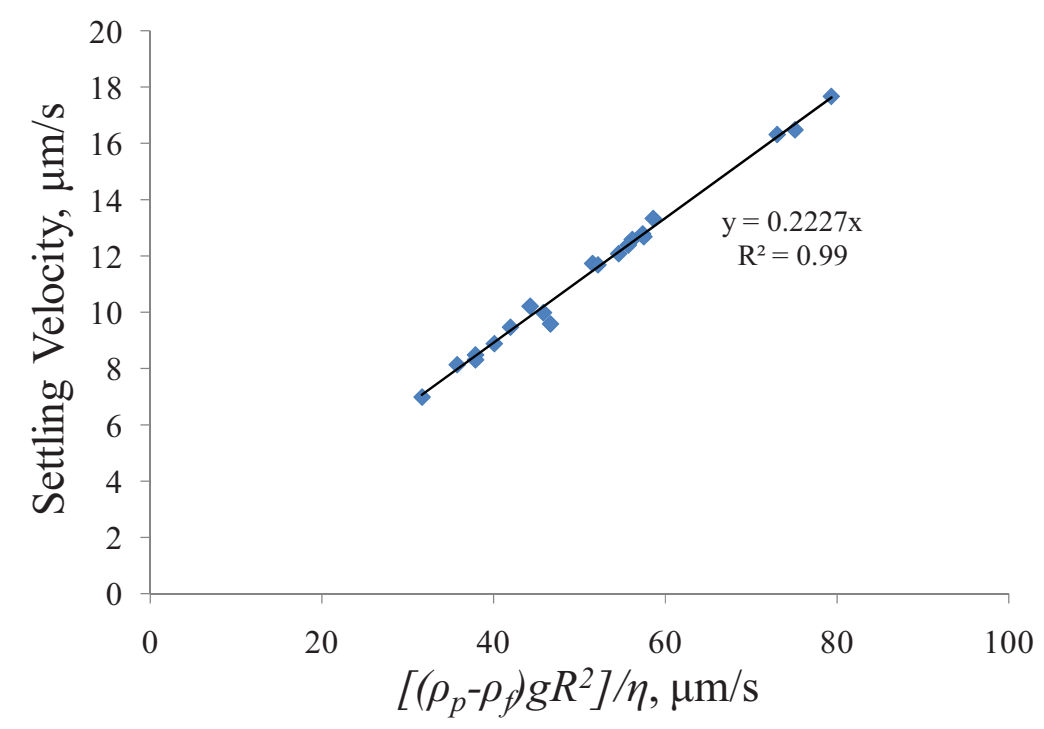

Figure 11.5. Linear Fit of Settling Velocity vs. $\left[\left(\rho_{p}-\rho_{f}\right) g R^{2}\right] / \eta$ for Glass Beads 
Table 11.1. Settling Velocities and Shape Factors for Glass Beads of Different Size and Density in Different Viscosity Oils

\begin{tabular}{cccccc}
\hline & $\begin{array}{c}\text { Bead } \\
\text { Diameter } \\
(\mu \mathrm{m})\end{array}$ & $\begin{array}{c}\text { Bead Density } \\
\left(\mathrm{g} / \mathrm{cm}^{3}\right)\end{array}$ & $\begin{array}{c}\text { Oil Viscosity } \\
(\text { Pa.s })\end{array}$ & $\begin{array}{c}\text { Settling } \\
\text { Velocity } \\
(\mu \mathrm{m} / \mathrm{s})\end{array}$ & Shape Factor \\
\hline 1 & 190 & 4.1741 & 4.840 & 13.3500 & 0.2278 \\
\hline 2 & 186 & 4.1741 & 4.840 & 12.6000 & 0.2243 \\
\hline 3 & 188 & 4.1741 & 4.840 & 12.8000 & 0.2231 \\
\hline 4 & 168 & 4.1741 & 4.840 & 10.0000 & 0.2182 \\
\hline 5 & 142 & 2.4842 & 1.025 & 16.3333 & 0.2236 \\
\hline 6 & 144 & 2.4842 & 1.025 & 16.5000 & 0.2196 \\
\hline 7 & 148 & 2.4842 & 1.025 & 17.6875 & 0.2229 \\
\hline 8 & 124 & 2.4842 & 1.025 & 12.4000 & 0.2226 \\
\hline 9 & 82 & 4.1741 & 1.025 & 11.7500 & 0.2279 \\
10 & 74 & 4.1741 & 1.025 & 9.4857 & 0.2260 \\
11 & 78 & 4.1741 & 1.025 & 9.6000 & 0.2193 \\
12 & 76 & 4.1741 & 1.025 & 10.2286 & 0.2168 \\
\hline 13 & 126 & 2.4842 & 1.025 & 12.7000 & 0.2185 \\
\hline 14 & 120 & 2.4842 & 1.025 & 11.7000 & 0.2219 \\
\hline 15 & 64 & 2.4842 & 0.480 & 7.0000 & 0.2209 \\
\hline 16 & 70 & 2.4842 & 0.480 & 8.5000 & 0.2242 \\
\hline 17 & 70 & 2.4842 & 0.480 & 8.3250 & 0.2196 \\
\hline 18 & 84 & 2.4842 & 0.480 & 12.1000 & 0.2217 \\
\hline 19 & 68 & 2.4842 & 0.480 & 8.1500 & 0.2278 \\
\hline 20 & 72 & 2.4842 & 0.480 & 8.9000 & 0.2219 \\
\hline & & & & & \\
\hline
\end{tabular}

Figure 11.6 shows the linear fit of settling velocity vs. $\left[\left(\rho_{p}-\rho_{f}\right) g R^{2}\right] / \eta$ for individual spinel crystals and spinel agglomerates. The fitted shape factor for individual crystals was slightly higher than that for glass beads. Authors Happel and Brenner used a Stokes shape factor correlated to the particle sphericity through the equation:

$$
k^{\prime}=0.843 \times \log \frac{\psi}{0.065}
$$

where the sphericity $\psi$ is defined as the ratio of the surface area of a sphere to the surface area of a particle with the same volume. If multiplied by $2 / 9$, this shape factor becomes the Stokes shape factor $k_{s}$ from Equation (11.3). The obtained spinel shape factor of 0.2246 was $7.6 \%$ higher than the theoretical Stokes shape factor of octahedrons $0.2087(\psi=0.846)$. This difference can be attributed to the higher scatter of data (smaller $\mathrm{R}^{2}$ value) resulting from irregularities in crystal shape (e.g., crystal elongation and surface defects) and differences in crystal orientation during settling. Variations in crystal geometry cause a divergence from the sphericity of an isometric octahedron. 


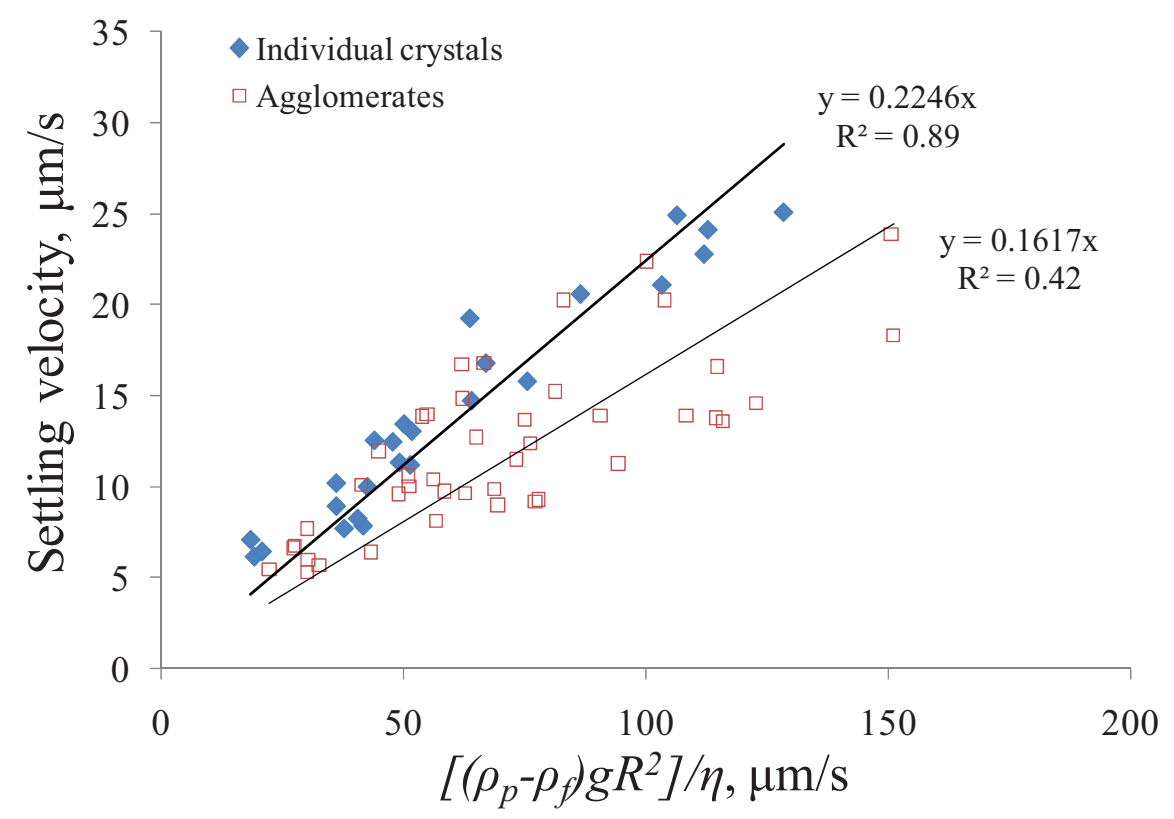

Figure 11.6. Linear Fit of Settling Velocity vs. $\left[\left(\rho_{p}-\rho_{f}\right) g R^{2}\right] / \eta$ for Single and Agglomerated Spinel Crystals

The data in Figure 11.6 show that the calculated shape factors for spinel agglomerates were widely scattered. Case-to-case variations in the number of agglomerated crystals, the degree of overlap, and the agglomerate orientation can explain this variance. In addition, multi-particle agglomerates likely contained holes or inter-pockets that slowed down their settling velocity. These features could not be detected from the shallow three-dimensional images, but were observed during SEM analysis of the precipitated spinel crystals (Figure 11.2). Also, when only two- or three-particle agglomerates were observed, the determined shape factors were within $10 \%$ of that of the single spinel crystals. Figure 11.7 shows examples of a two-particle agglomerate and a multi-particle agglomerate with Stokes shape factors $\sim 0.2442$ and $\sim 0.1879$, respectively. The fitted shape factor for multiparticle agglomerates was more than $25 \%$ lower than for glass beads. This can be attributed to larger drag forces caused by a higher surface area-to-volume ratio. Spheres have the smallest surface area for all shapes of a given volume and at low Reynolds numbers experience a minimum amount of resistance as they travel through a fluid. A decrease in the sphericity, corresponding to an increase in the surface area-to-volume ratio, results in an increase in the surface drag and a decrease in velocity for an object travelling through a given fluid. The obtained shape factors were implemented in Equation 11.2 and used to calculate hindered settling velocities of particles in concentrated suspensions. 

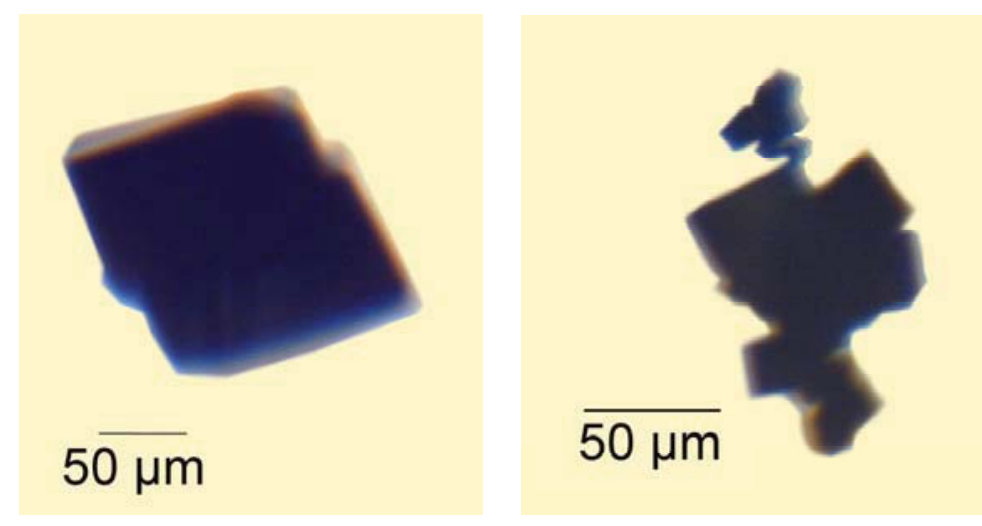

Figure 11.7. Two (A) and Multiple-Particle (B) Agglomerates of Spinel Crystals

\subsection{Hindered Settling}

For particle volume concentrations as low as $1 \%$, the hindered settling effects, such as particleparticle interactions and upward flow due to fluid displacement as particles travel downwards, cause a noticeable slowing in the settling velocity. One of the most widely used empirical relationships to calculate the hindered settling velocity in monodispersed concentrated suspensions is the Richardson-Zaki equation:

$$
v^{\prime \prime}=v\left(1-C_{0}\right)^{n}
$$

where $C_{0}$ is the volume fraction of particles in the suspension, and $\mathrm{n}$ is an exponent that for the Reynolds number $\operatorname{Re} \leq 0.2$ is approximately equal to 4.65 . Richardson and Zaki also reported in their pioneer experimental work that $\mathrm{n}$ decreases from 4.65 to 2.39 as Re increases. Figure 11.8 shows that the Richardson-Zaki hindered settling coefficient, $k_{h}=\left(1-C_{0}\right)^{4.65}$, decreases rapidly with increasing $C_{0}$ at low volume fractions of solids.

To study hindered settling, the Ba-Ca silicate glass beads and spinel crystal suspensions with volume concentrations ranging from 1 to $4 \%$ were prepared. The particles were uniformly dispersed in the viscosity oil by placing the container on a double barrel roller for approximately $1 / 2$ hour. Then, the suspension was poured into a cuvette that was transferred into a vacuum desiccator to remove air bubbles that were generated during mixing and pouring. The tested particle-size distributions, densities, viscosities, and volume concentrations of suspensions are shown in Table 11.2. To prevent electrostatic charge accumulation due to particle-particle and particle-wall interactions, the anti-static sheets were wrapped around the bottles during mixing, and they covered the side walls of the cuvette during settling experiments. 


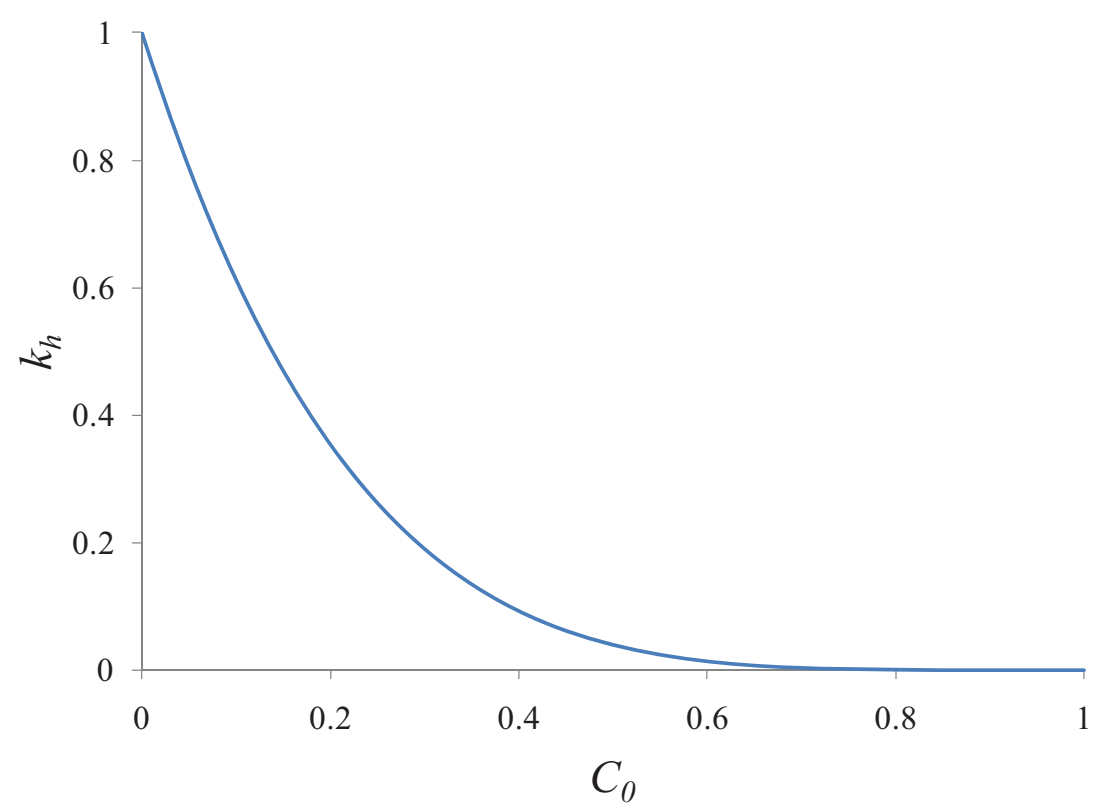

Figure 11.8. Richardson-Zaki Hindered Settling Coefficient vs. Volume Fraction of Solids

A digital camera mounted on a tripod was used to capture the successive images of the settling front. The collected images were analyzed with Clemex Image Analysis Software (CIAS) to determine the position of the settling front and the concentration profile of particles. The concentration profile was determined by dividing the suspension in the cuvette into a series of equally sized rectangles $(5.6 \mathrm{~mm}$ long; $1 \mathrm{~mm}$ wide). Each rectangle was evaluated with CIAS for the particle void fraction as shown in Figure 11.9. The rate of clarification (increase in the particle void fraction) at any given rectangle was found by plotting the void fraction in that rectangle against the time at which the image was taken.

Table 11.2. Characteristics of Particle Suspensions for the Hindered Settling Experiments

\begin{tabular}{ccccc}
\hline Particles & Size Distribution, $\mu \mathrm{m}$ & Density, $\mathrm{g} / \mathrm{cm}^{3}$ & Viscosity, Pa.s & Concentration of Suspensions, vol\% \\
\hline Glass Beads & $150-180$ & 4.1741 & 4.84 & $1.0 ; 1.5 ; 2.5 ; 3.0 ; 4.0$ \\
Glass Beads & $67-80$ & 4.1741 & 4.84 & $1.0 ; 2.0 ; 3.0$ \\
Spinel Crystals & $149-212$ & 5.3954 & 5.08 & $0.5 ; 1 ; 1.5$ \\
\hline
\end{tabular}


A

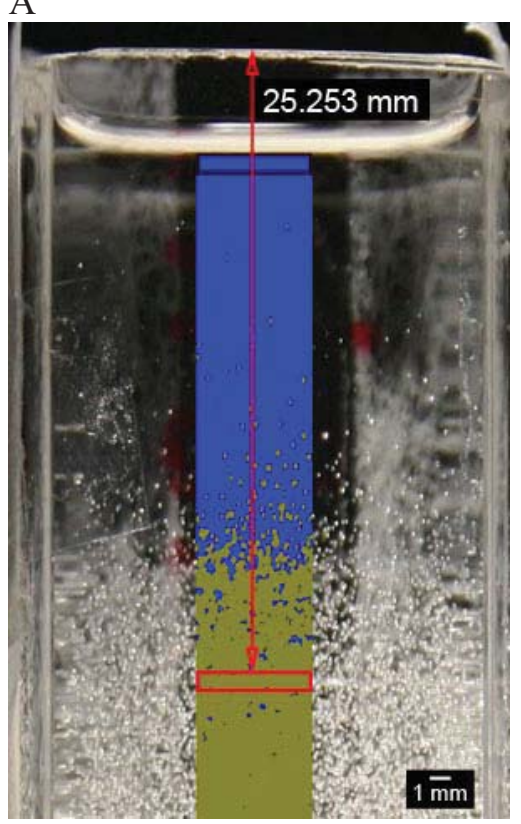

B

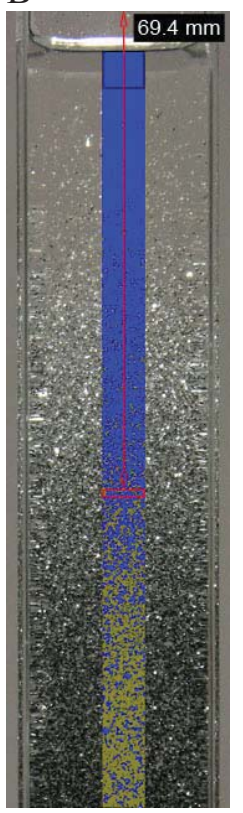

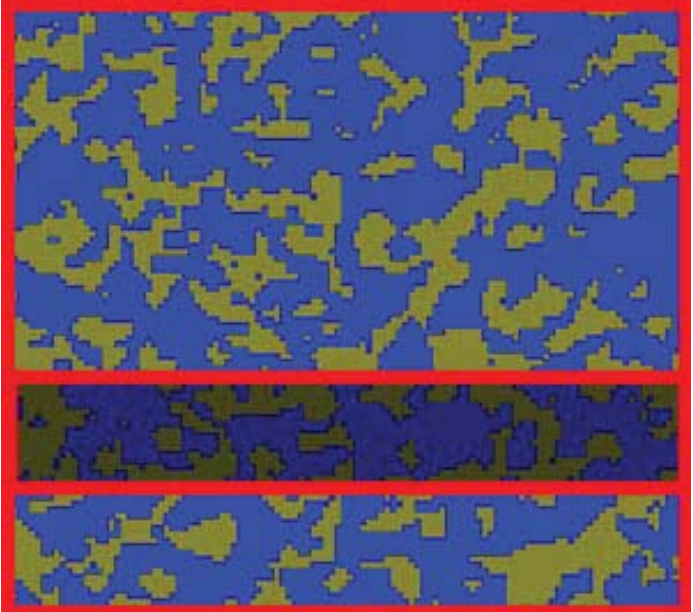

Figure 11.9. Image Threshold Analysis of Tested Suspensions (particle voids - blue; glass beads and crystals - green): A) 1 vol $\%$ of 150 - to $180-\mu \mathrm{m}$ Glass Beads in 4.84 Pa.s oil, B) 1 vol\% of 149- to 212- $\mu \mathrm{m}$ Spinel Crystals in 5.08 Pa.s Oil, and C) Detail of Rectangle from Image B

Three distinct regions were identified in the polydispersed suspensions: 1) the clarified fluid at the top of the settling vessel, 2) the transition region where the concentration varied from nearly clarified to more concentrated, and 3) the region of the suspension containing all particles at their initial concentrations. The settling front for spinel crystal and glass bead suspensions was defined by a near particle concentration discontinuity inside at the end of the transition region, respectively. Figure 11.10 shows optical images providing the location of the settling front for 1-vol\% silicone oil suspensions of glass beads and spinel crystals. Figure 11.11 and Figure 11.12 show the change of the particle void fraction over time and distance and the determined positions of the settling front (distance from the top of the cuvette) for these suspensions. The obtained distances were then plotted against time as shown in Figure 11.13. The slopes of fitted lines corresponded to the hindered settling velocities ( $v ")$ of suspensions. The settling velocities of single particles (v) were determined by extrapolating the linear fitted lines of hindered settling velocities for different volume fraction suspensions $\left(\mathrm{C}_{0}\right)$ to zero (infinitely diluted suspensions). Then, we obtained the Richardson-Zaki exponent $\mathrm{n}$ for particles from the slopes of linear fit of data $\left[\log \left(1-\mathrm{C}_{0}\right) \mathrm{vs} . \log \left(\mathrm{v}^{\prime \prime} / \mathrm{v}\right)\right]$ shown in Figure 11.14. The experimentally determined value of the exponent $\mathrm{n}$ for spinel crystals was $\sim 2.9$ and for glass beads $\sim 3.6$. These values are somewhat lower than 4.65 , probably due to different sizes, structures, shapes, and roughness of agglomerates, which may affect the interaction between the agglomerates and the fluid. In any case, we have confirmed that the Richardson-Zaki equation can be an appropriate and very useful tool for predicting the settling behavior of sedimenting concentrated suspensions and more specifically, for predicting the settling of spinel crystals in the glass discharge riser of the HLW melter. 

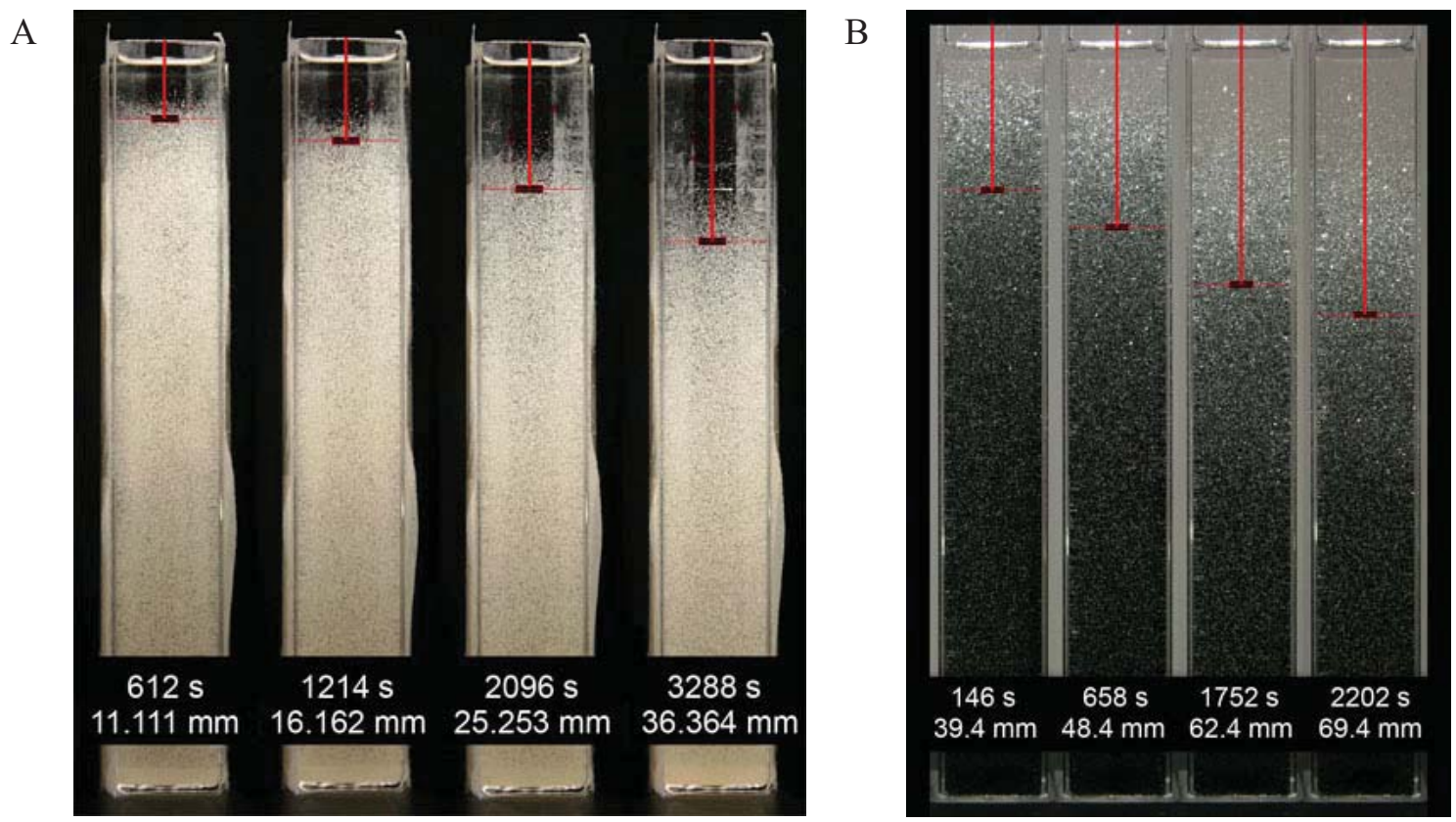

Figure 11.10. Settling Front Movement for $1-\mathrm{vol} \%$ Silicone Oil Suspensions of Glass Beads and Spinel Crystals: A) 150 - to $180-\mu \mathrm{m}$ Glass Beads in 4.84 Pa.s oil, B) 149- to 212- $\mu \mathrm{m}$ Spinel Crystals in 5.08 Pa.s Oil

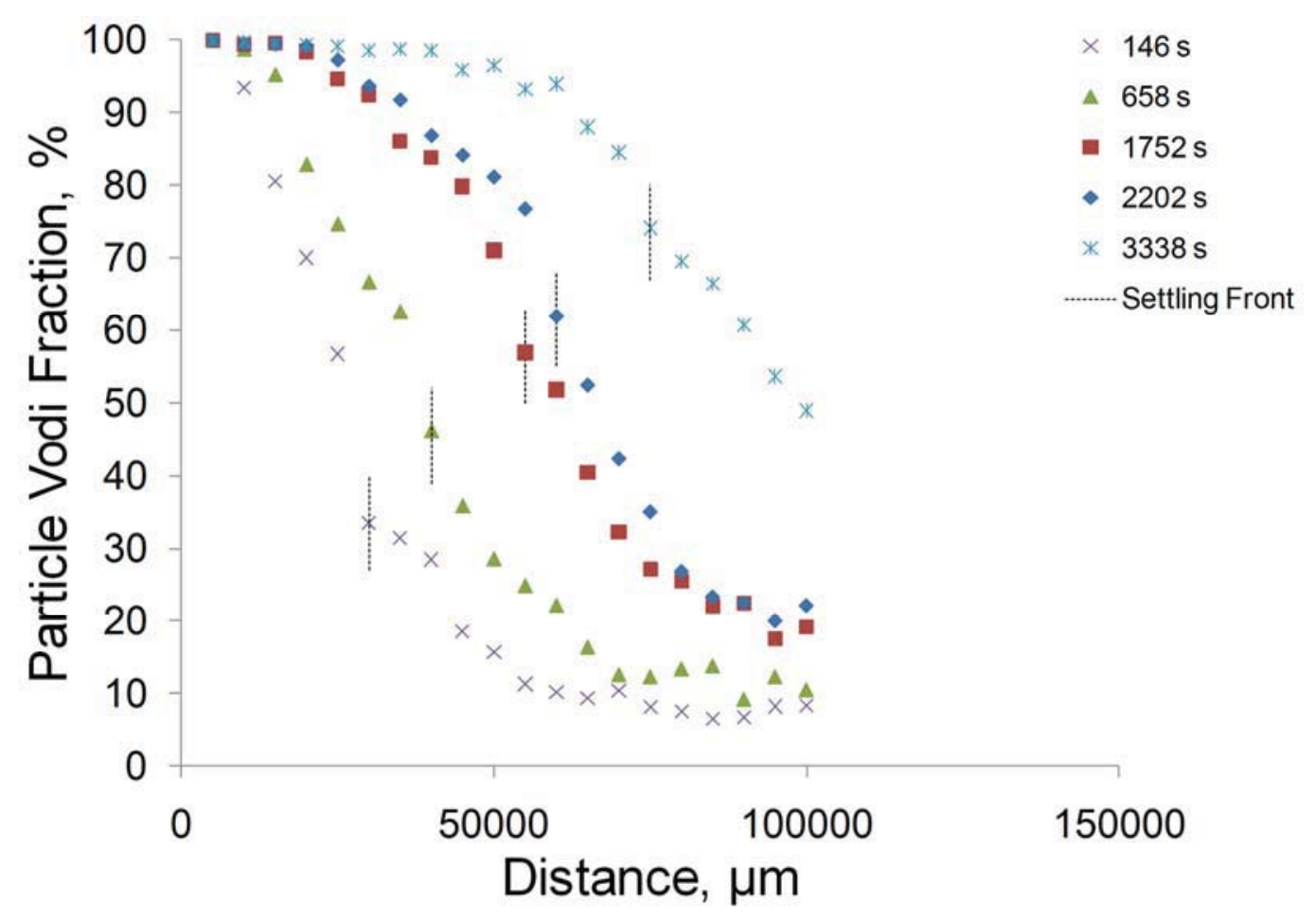

Figure 11.11. Change of Particle Void Fraction over Time and Distance and Determined Positions of Settling Front for 1 vol\% of 149- to 212- $\mu$ m Spinel Crystals in 5.08 Pa.s Oil 


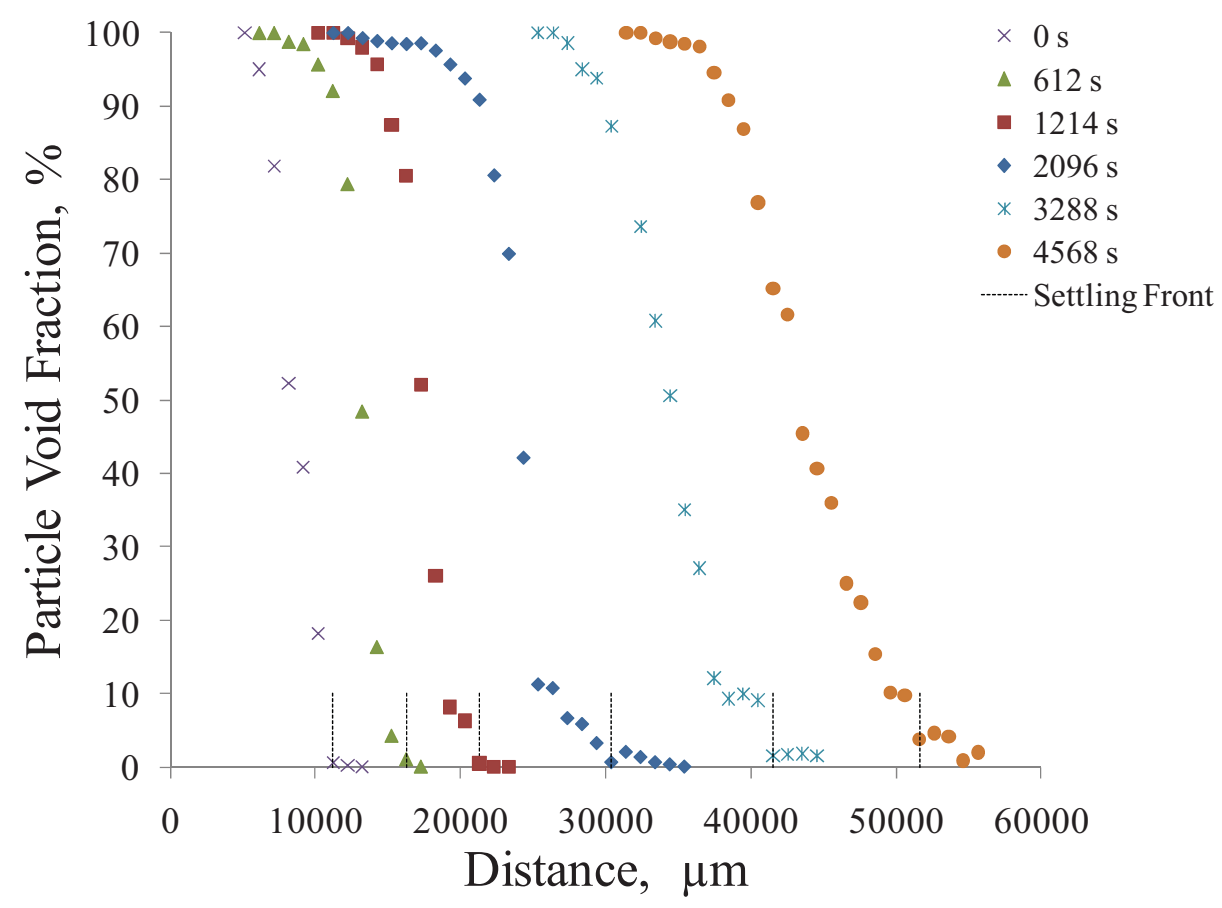

Figure 11.12. Change of Particle Void Fraction over Time and Distance and Determined Positions of Settling Front for 1-vol\% Oil (4.84 Pa.s) Suspension of 150- to 180- $\mu \mathrm{m}$ Glass Beads

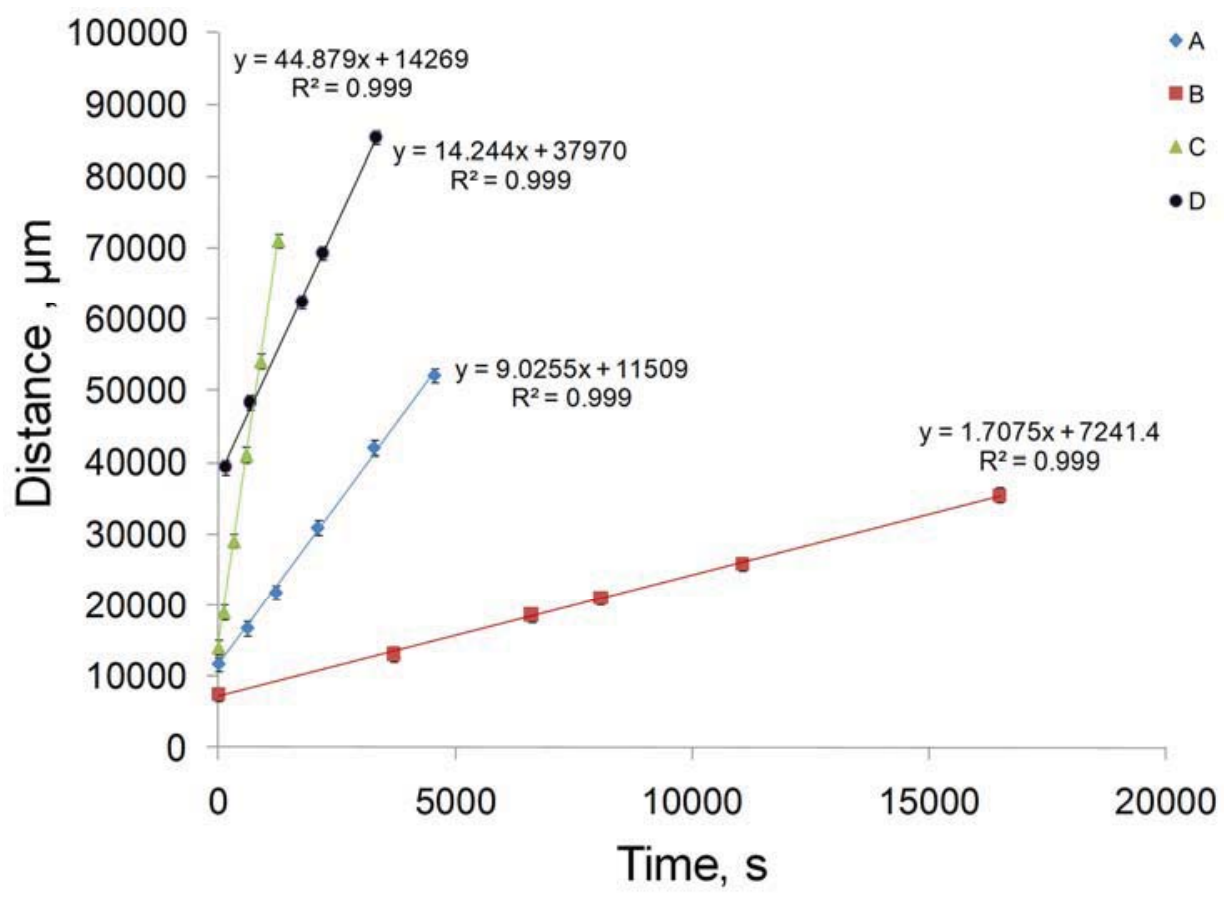

Figure 11.13. Distance vs. Time for 1 vol\% Silicone Oil Suspensions of Glass Beads and Spinel Crystals: A) 150 - to $180-\mu \mathrm{m}$ Glass Beads in 4.84 Pa.s Oil, B) 67- to $80-\mu \mathrm{m}$ Glass Beads in 4.84 Pa.s Oil, C) 150- to 180- $\mu \mathrm{m}$ Glass Beads in 0.98 Pa.s Oil, and D) 149 - to $212-\mu \mathrm{m}$ Spinel Crystals in 5.08 Pa.s Oil 


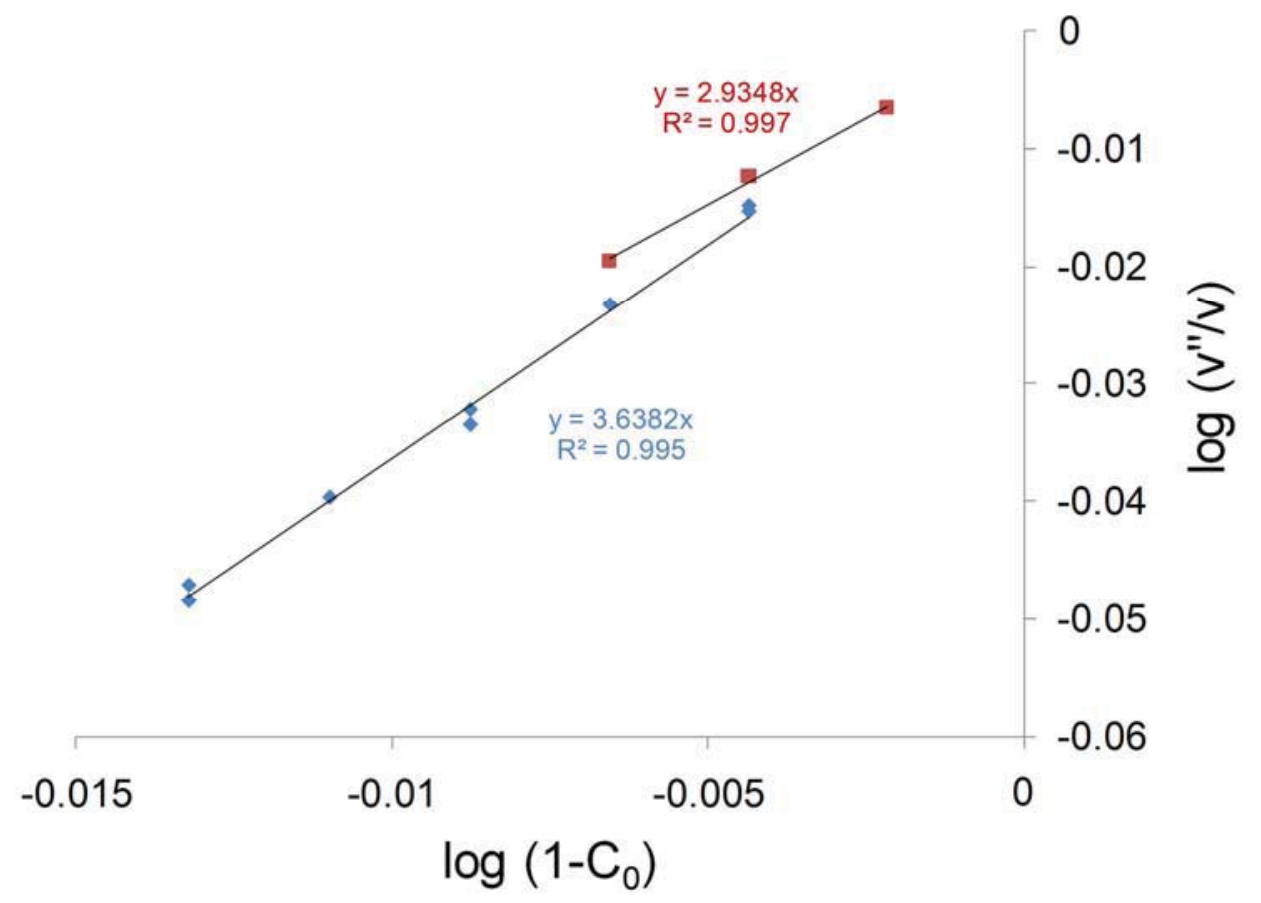

Figure 11.14. Plot of $\log \left(1-C_{0}\right)$ vs. $\log \left(v^{\prime \prime} / v\right)$. Slopes of fitted lines correspond to Richardson-Zaki exponent $n$. 

PNNL-20072

\section{Distribution}

No. of

Copies

2 Catholic University of America

Vitreous State Laboratory, 620 Michigan

Ave., N.E., Washington, D. C. 20064

Wing Kot

Ian Pegg

1 Sharon Mara

Savannah River National Laboratory

773-A, Aiken, SC 29808

1 David Peeler

Savannah River National Laboratory

999-W, Aiken, SC 29808

1 Larry Petkus

Waste Treatment and Immobilization Plant 2435 Stevens Center Place, Richland, WA 99354

1 Steve Schneider

U.S. Department of Energy

EM-31, 19901 Germantown Road,

Germantown, MD 20874
No. of

Copies

\section{Local Distribution}

Pacific Northwest National Laboratory

Paul Bredt

K9-69

Mona Champion

$\mathrm{K} 3-52$

Jarrod Crum

K6-24

Pavel Hrma

K6-24

Brad Johnson

K6-24

Dong Kim

K6-24

Albert Kruger

H6-60

Josef Matyas (3)

K6-24

Billie Mauss

H6-60

John McCloy

K6-24

Reid Peterson

P7-27

Loni Peurrung

K9-09

Richard Pokorny

K6-24

Gene Ramsey

B1-55

Brian Riley

K6-24

Joe Ryan

K6-24

Mike Schweiger

K6-24

Gary Sevigny

P7-27

Gary Smith

H6-60

John Vienna

K6-24 




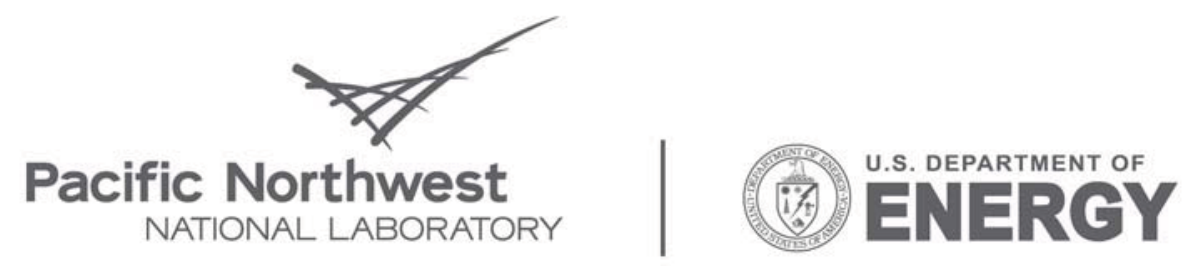

Proudly Operated by Battelle Since 1965

902 Battelle Boulevard

P.O. Box 999

Richland, WA 99352

1-888-375-PNNL (7665)

www.pnl.gov 